Pacific Journal of Mathematics

VISIBILITY MANIFOLDS 


\section{VISIBILITY MANIFOLDS}

\section{P. EberLein AND B. O'NeILL}

Several of the basic features of automorphic function theory-notably the notion of limit set-can be extended to apply to the study of Riemannian manifolds $M$ of nonpositive curvature. Under somewhat stronger curvature conditions (e.g. $K \leqq c<0) M$ is called a Visibility manifold. For such manifolds there results a classification into three types: parabolic, axial, and fuchsian. This trichotomy is closely related to many of the most basic topological and geometric properties of $M$, and such relationships will be examined in some detail. For example, the trichotomy may be expressed in terms of the number (suitably counted) of closed geodesics in $M$, namely: 0,1 , or $\infty$. As to methodology: the conventional analytic machinery of $C^{\infty}$ Riemannian geometry is used, at least initially; however, at many crucial points it will be the qualitative behavior of geodesics (ala Busemann) that is important.

In the late nineteenth century Poincaré discovered an important link between geometry and automorphic function theory, namely that the open disk $P$ in the complex plane admits a Riemannian metric such that (1) $P$ has constant negative curvature and gives a model for the noneuclidean geometry of Bolyai and Lobachevsky; and (2) the orientation preserving isometries of $P$ are exactly the linear fractional transformations that preserve $P$.

Automorphic function theory makes essential use of an extrinsic feature of the disk: its boundary circle $S^{1}$ in the plane. For example, a linear fractional transformation $\varphi$ as in (2) that has no fixed points in $P$ must have either one or two fixed points in $S^{1}$. Also, if $D$ is a properly discontinuous group of such transformations then the accumulation points of any orbit $D(p), p \in P$, form a closed $D$-invariant subset $L(D)$ of $S^{1}$ called the limit set of $D$. Analysis of the limit set $L(D)$ and of the fixed points of the elements of $D$ gives extensive information about the Riemann surface $M=P / D$. When the Poincaré metric was introduced on $P$ this approach also yielded properties of the geodesic flow on surfaces $M$ of constant negative curvature. But since the method was tied to complex analysis it was not clear how to extend it to manifolds of higher dimension and variable curvature.

Our object in this paper is to study Riemannian manifolds of sectional curvature $K \leqq 0$ by generalizing, or rather geometrizing, some of these basic features of automorphic function theory. Thus 
the role of the disk $P$ is taken by an arbitrary complete simplyconnected $n$-manifold $H$ with $K \leqq 0$. We begin by defining points at infinity for $H(\S 1)$. With a suitable topology, the cone topology ( $\S 2$, these constitute a boundary sphere $H(\infty)$ such that $\bar{H}=H \cup H(\infty)$ is a closed $n$-cell. An isometry $\varphi$ of $H$ extends naturally to a homeomorphism of $\bar{H}$; thus if $D$ is a properly discontinuous group of isometries on $H$, we obtain as before a closed $D$-invariant limit set $L(D)$ in $H(\infty)$. It is natural then to consider what influence the limit set and the action of $D$ on $H(\infty)$ have on the quotient manifold $M=H / D$. For this influence to be decisive it is at present necessary to impose geometrical restrictions on $M=H / D$ beyond merely $K \leqq 0$. While the standard condition $K \leqq c<0$ would suffice, we prefer to express the restrictions in terms of one and occasionally two axioms on the geodesics of $H(\S 4)$. These hold under curvature conditions ( $\$ 5$ ) distinctly weaker than $K \leqq c<0$. We use them, however, not merely for the sake of generalization, but because they result in much simpler proofs, with qualitative (synthetic) arguments replacing quantitative (analytic) ones. At this and other points we have been influenced by the ideas of Busemann [4].

If $H$ satisfies Axiom 1, then its isometries share many of the properties of linear fractional transformations $(\S \S 6,7)$, notably that if $\phi$ has no fixed points in $H$ then it has exactly one or two in the boundary sphere $H(\infty)$.

Another useful topology on $\bar{H}=H \cup H(\infty)$ derives from the classical notion of horocycle $(\S 3)$. There is a corresponding limit set $L_{h}(D) \subseteq H(\infty)$ which is, for example, closely related to the lengthminimizing properties of geodesics in $M=H / D$.

When $H$ satisfies Axiom 1 we call $M=H / D$ a Visibility manifold; this class includes all complete manifolds of curvature $K \leqq c<$ 0 . Both the cone and horocycle topologies are used to define points at infinity for an arbitrary Visibility manifold $M$ and topologize $\bar{M}=$ $M \cup M(\infty)$ in a natural way (\$10) that generalizes the construction of $\bar{H}$.

By investigating limit sets we divide all Visibility manifolds into three types: parabolic, axial, or fuchsian. The concepts outlined above are, as we have said, closely related to many fundamental topological and geometric properties of $M$; for example, closed geodesics ( $\S 8$ ), fundamental group $(\S 9)$, ends $(\S 10)$ and convex functions $(\S 11)$. In particular the following picture emerges of the three types mentioned above:

A parabolic manifold $M$ is a topological product $N \times R^{1}$ with each $\{n\} \times R^{1}$ a minimizing geodesic. $M$ has no closed geodesics, and $L(D)$ is a single point. Despite its simple geometric structure $M$ can have a remarkably wide variety of fundamental groups. Para- 
bolic manifolds are characterized by the fact that they admit a convex function without minimum.

An axial manifold $M$ is a smooth vector bundle over a circle, hence is diffeomorphic to either $S^{1} \times R^{n-1}$ or $B \times R^{n-2}$, where $B$ is the Möbius band. $M$ has a unique equivalence class $A$ of closed geodesics. All members of $A$ are simply closed, (though possibly of different periods), and $A$ reduces to a single closed geodesic when Axiom 2 holds. The fundamental group of $M$ is infinite cyclic, and $L(D)$ is two points.

A Visibility manifold $M$ is fuchsian if $L(D)$ has more than two points, for example if $M$ is compact or has more than two ends. A fuchsian manifold $M$ has infinitely many equivalence classes of closed geodesics. Although $M$ may have many different topological structures its fundamental group must satisfy severe algebraic conditions. We phrase these abstractly in terms of the notion of monic group, a class of groups including solvable groups, nontrivial product groups, etc.

Our axiomatic approach has applications to geodesic flows [7]. The present paper developed from joint work by one of us with R. L. Bishop, and we are grateful to him for many valuable insights.

1. Points at infinity. For any Riemannian manifold $M$ we denote the Riemannian structure by $\langle$,$\rangle , the Riemannian metric by$ $d$. If $p \in M$ let $S(p)$ be the unit sphere in the tangent space $M_{p}$; let $S M$ be the unit tangent bundle with projection $\mu: S M \rightarrow M$. If $v, w \in$ $S(p)$, the angle $\theta=\Varangle(v, w)$ is the unique number $0 \leqq \theta \leqq \pi$ such that $\langle v, w\rangle=\cos \theta$. If $M$ is complete and $v \in S M$ let $\gamma_{v}: R \rightarrow M$ be the geodesic such that $\gamma_{v}^{\prime}(0)=v$. Throughout this paper all geodesics have unit speed and unless otherwise indicated are defined on the entire real line. A geodesic segment is a geodesic defined on a compact interval.

A Hadamard manifold $H$ is a complete, simply connected Riemannian manifold of dimension $n \geqq 2$ having sectional curvature $K \leqq 0$. $H$ will always denote a Hadamard manifold. We shall assume the basic information about such manifolds [2, 13]. If $p \neq q$ in $H$ let $\gamma_{p q}$ be the unique (unit speed) geodesic such that $\gamma_{p q}(0)=p$ and $\gamma_{p q}(t)=q$ where $t=d(p, q)$. The angle $\Varangle_{p}(m, n)$ subtended by points $m, n$ of $H$ at a distinct point $p$ is defined to be $\Varangle\left(\gamma_{p m}^{\prime}(0), \gamma_{p n}^{\prime}(0)\right)$. Any three non-collinear points of $H$ determine a geodesic triangle and we have:

(1) Law of Cosines: $c^{2} \geqq a^{2}+b^{2}-2 a b \cos \theta$ where $a, b, c$ are the sides and $\theta$ is the angle opposite $c$.

(2) Angle sum: the sum of the interior angles of a triangle is at most $\pi$.

(3) Double law of cosines (obtained from applying the law of 
cosines twice): A geodesic triangle with sides $a, b, c$ and angles $\alpha$, $\beta$ and $\gamma$ opposite the corresponding sides satisfies $c \leqq b \cos \alpha+a \cos \beta$.

We make use of the following consequence of the law of cosines: if $\left\{p_{n}\right\},\left\{q_{n}\right\}$ and $\left\{r_{n}\right\}$ are sequences in $H$ such that $d\left(p_{n}, q_{n}\right) \rightarrow \infty$ but $d\left(q_{n}, r_{n}\right) \leqq A$ as $n \rightarrow \infty$, then $\Varangle_{p_{n}}\left(q_{n}, r_{n}\right) \rightarrow 0$.

We summarize briefly the basic facts about asymptotes. The logical order differs slightly from [3] and [4] since we replace the classical definition by:

Definition 1.1. Geodesics $\alpha$ and $\beta$ in a Hadamard manifold $H$ are asymptotic provided there exists a number $c>0$ such that $d(\alpha t, \beta t) \leqq c$ for all $t \geqq 0$.

The following consequences are immediate:

(1) If $\alpha$ and $\beta$ are asymptotic, then so are orientation-preserving unit speed reparametrizations of $\alpha$ and $\beta$.

(2) The asymptote relation is an equivalence relation on the set of all geodesics in $H$; the equivalence classes are called asymptote classes.

(3) If asymptotic geodesics in $H$ have a point in common, then they are the same but for parametrization (by the law of cosines).

It follows from (2) and (3) that there is at most one geodesic starting at a given point and asymptotic to a given geodesic. Proposition 1.2 below will yield:

(4) Given a geodesic $\alpha$ and a point $p \in H$ there exists a unique geodesic $\beta$ such that $\beta(0)=p$ and $\beta$ is asymptotic to $\alpha$.

We assume the facts about convex functions contained in [3]. Convex functions are presumed to be only continuous unless differentiability is explicitly mentioned. The following results will be used often:

(a) If $\alpha, \beta$ are geodesics of $H$, then the function $t \rightarrow d(\alpha t, \beta t)$ is $C^{\infty}$ convex $\left(f^{\prime \prime} \geqq 0\right)$. If $\alpha$ and $\beta$ are asymptotic, then $d(\alpha t, \beta t)$ is monotone decreasing in $t$.

(b) The functions $t \rightarrow d(\alpha t, \beta)$ and $t \rightarrow d(\beta t, \alpha)$ are continuous convex functions, and in fact if $\alpha$ and $\beta$ are asymptotic then

$$
\begin{aligned}
\lim _{t \rightarrow \infty} d(\alpha t, \beta) & =\lim _{t \rightarrow \infty} d(\beta t, \alpha)=d(\alpha, \beta)=d(\alpha R, \beta R) \\
& =\inf \{d(\alpha s, \beta t): s, t \in R\} .
\end{aligned}
$$

Proposition 1.2. Let $\alpha$ be a geodesic in $H$ and let $\left\{p_{n}\right\} \rightarrow p$ in $H,\left\{t_{n}\right\} \rightarrow \infty$ in $R$. If $\beta_{n}$ is the geodesic from $p_{n}$ to $\alpha\left(t_{n}\right)$ then $\beta_{n}^{\prime}(0)$ converges to a vector $v \in S(p)$, and $\beta=\gamma_{v}$ is asymptotic to $\alpha$.

Proof. Let $c>0$ be a number such that $d\left(p_{n}, \alpha 0\right) \leqq c$ for all $n$. 
Let $s_{n}$ be the number such that $\beta_{n}\left(s_{n}\right)=\alpha\left(t_{n}\right)$. By the triangle inequality $\left|s_{n}-t_{n}\right| \leqq d\left(p_{n}, \alpha 0\right) \leqq c$. In particular $\left\{s_{n}\right\} \rightarrow \infty$. Now fix $s \geqq 0$, and let $n$ be so large that $s \leqq s_{n}$.

Since the function $t \rightarrow d\left(\beta_{n} t, \alpha t\right)$ is convex we have $d\left(\beta_{n} s, \alpha s\right) \leqq$ $\max \left\{d\left(\beta_{n} 0, \alpha 0\right), d\left(\beta_{n} s_{n}, \alpha s_{n}\right)\right\}$. But $d\left(\beta_{n} s_{n}, \alpha s_{n}\right)=d\left(\alpha t_{n}, \alpha s_{n}\right)=\left|t_{n}-s_{n}\right| \leqq$ $c$ and $d\left(\beta_{n} 0, \alpha 0\right) \leqq c$, since $p_{n}=\beta_{n}(0)$. Thus $\left(^{*}\right) d\left(\beta_{n} s, \alpha s\right) \leqq c$ for all $s \geqq 0$. Some subsequence of $\left\{\beta_{n}^{\prime}(0)\right\}$ converges to a vector $v \in S(p)$. By continuity of the exponential map, $\left({ }^{*}\right)$ implies that $d\left(\gamma_{v} s, \alpha s\right) \leqq c$ for all $s \geqq 0$, that is, $\gamma_{v}$ is asymptotic to $\alpha$. By (5) above this asymptote is unique and every convergent subsequence of $\left\{\beta_{n}^{\prime}(0)\right\}$ has the limit $v$.

The complete manifolds $M$ of dimension $n \geqq 2$ and sectional curvature $K \leqq 0$ are precisely the quotient manifolds $H / D$ where $H$ is a Hadamard manifold and $D$ is a properly discontinuous group of isometries of $H$. We shall study $H / D$ through the action of $D$ on $H$ and the geometric properties of $H$ at infinity. The main tool used is the notion of a point at infinity of $H$ which we define to be simply an asymptote class of geodesics of $H$. Let $H(\infty)$ be the set of points of infinity of $H$, and let $\bar{H}=H \cup H(\infty)$. If $H$ is $n$-dimensional hyperbolic space, the open unit ball in $R^{n}$ with the Poincaré metric, then $H(\infty)$ is the bounding sphere $S^{n-1}$. This example in fact exhibits and is the motivation for the geometric properties that we shall consider.

If $\alpha:(-\infty, \infty) \rightarrow H$ is a geodesic, let $\alpha(\infty)$ be the asymptote class of $\alpha$ and let $\alpha(-\infty)$ be the asymptote class of the reverse curve $t \rightarrow$ $\alpha(-t)$. The resulting function $\alpha:[-\infty, \infty] \rightarrow \bar{H}$ is the asymptotic extension of $\alpha$. Note that $\alpha(-\infty) \neq \alpha(\infty)$, since $\alpha$ realizes the distance between any two of its points. If $x \in H(\infty)$ we write either $\alpha(\infty)=x$ or $\alpha \in x$ depending upon the context. In this notation the remark (4) preceding Proposition 1.2 says that given $p$ in $H$ and $x$ in $H(\infty)$ there exists a unique geodesic $\gamma_{p x}$ such that $\gamma_{p x}(0)=p$ and $\gamma_{p x}(\infty)=x$. Roughly speaking, there is a unique geodesic joining any finite point to any infinite point.

Similarly if $\varphi$ is an isometry of $H$ and $x$ a point in $H(\infty)$ we set $\varphi(x)=(\varphi \circ \alpha)(\infty)$, where $\alpha$ is any geodesic representing $x$. Since asymptotes are preserved under isometries we obtain a well-defined function $\varphi: \bar{H} \rightarrow \bar{H}$ which is bijective and carries $H(\infty)$ into itself.

In the nonsimply connected case $M=H / D$, geodesics $\alpha$ and $\beta$ are asymptotic if they have lifts into $H$ that are asymptotic in the sense of Definition 1.1. This is equivalent to the existence of a homotopy from $\alpha$ to $\beta$ whose transverse curves from $\alpha(t)$ to $\beta(t)$ have bounded length for $t \geqq 0$. Clearly it is also an equivalence relation. Let $A(M)$ be the set of all asymptote classes of geodesics of $M$. If $x \in H(\infty)$ let $\pi_{a}(x)$ be the element of $A(M)$ represented by 
$\pi \circ \alpha$, where $x$ is represented by the geodesic $\alpha$. Then $\pi_{a}: H(\infty) \rightarrow$ $A(M)$ is well-defined and surjective, and for any $y$ in $A(M)$ the counterimage $\pi_{a}^{-1}(y)$ is an orbit of $D$ in $H(\infty)$. Briefly: $A(M)=$ $H(\infty) / D$.

There is more than one topology of interest on $\bar{H}$. We shall say that topology $\tau$ on $\bar{H}$ is admissible if it satisfies the following four conditions:

(1) Closure property: the topology on $H$ induced by $\tau$ is the original topology of $H$, and $H$ is a dense open set of $\bar{H}$.

(2) Geodesic extension property: if $\alpha$ is any geodesic of $H$ then its asymptotic extension is continuous.

(3) Isometric extension property: if $\varphi$ is any isometry of $H$ then its asymptotic extension is continuous (and hence a homeomorphism by a functorial argument).

(4) Intensive property: if $x \in H(\infty), V$ is a neighborhood of $x$, and $r>0$ is any positive number then there exists a neighborhood $U$ of $x$ such that $N_{r}(U)=\{q \in \bar{H}: d(q, U)<r\} \cong V$. Here we have extended the metric trivially so that $d(a, b)=\infty$ if $a \neq b$ and either point lies in $H(\infty)$. In fact this is the only continuous extension of the metric, assuming the geodesic extension property.

The inherited Euclidean topology on the closed unit ball in $R^{n}$ satisfies all four properties for the Poincare model of $n$-dimensional hyperbolic space.

The following easily checked fact will be useful.

Lemma 1.3. For each $x \in H(\infty)$ let $\mathscr{N}(x)$ be a collection of subsets of $\bar{H}$ such that (a) if $V \in \mathscr{N}(x)$ then $x \in V$ and $V \cap H$ is nonempty and open in $H$ and (b) if $V \in \mathscr{N}(x), W \in \mathscr{N}(y)$ and $z \in V \cap$ $W \cap H(\infty)$ there exists $U \in \mathscr{N}(z)$ such that $U \subseteq V \cap W$. Then there is a unique topology $\tau$ on $\bar{H}$ such that $\tau$ has the closure property and $\mathscr{N}(x)$ is a local basis for $\tau$ at each $x \in H(\infty)$.

We now construct the limit set of a group $D$ of isometries of $H$ relative to a suitable topology. Generally we will assume $D$ to be properly discontinuous, so for $p \in H$ the orbit $D(p)=\{\varphi(p): \varphi \in D\}$ will have no accumulation points in $H$. Depending on the topology used there will usually be accumulation points in $H(\infty)$.

Proposition 1.4. Let $\tau$ be a topology on $\bar{H}$ with the isometric extension and the intensive properties. Let $D$ be a group of isometries of $H$. Then

(1) The set of accumulation points of an orbit $D(p)$ in $H(\infty)$ is independent of the choice of $p \in H$ and is called the limit set $L_{\tau}(D)$ of $D$. 
(2) $L_{\tau}(D)$ is closed in $H(\infty)$ and is invariant under $\nu$ for each $\nu \in N(D)$, the normalizer of $D$ in the isometry group $I(H)$ of $H$.

(3) If $\tau$ is a $T_{1}$ topology, then $D$ is not properly discontinuous at any point of $L_{\tau}(D)$.

Proof. (1) Let $x \in \overline{D(p)} \cap H(\infty)$ and let $q \in H$. If $U$ is any neighborhood of $x$ then there exists a neighborhood $V$ of $x$ such that $N_{\varepsilon}(V) \leqq U$ where $\varepsilon=d(p, q)$. By assumption there exists $\varphi \in D$ such that $\varphi(p) \in V$. Hence $\varphi(q) \in U$ and it follows that $x \in \overline{D(q)} \cap H(\infty)$.

(2) Since $L_{\tau}(D)=\overline{D(p)} \cap H(\infty)$ it is clear that $L_{\tau}(D)$ is closed in $H(\infty)$. To prove invariance it suffices to prove that if $x \in \overline{D(p)} \cap$ $H(\infty)$ then $\nu(x) \in \overline{D(\nu p)} \cap H(\infty)$. If $U$ is any neighborhood of $\nu(x)$ then by the isometric extension property $\nu^{-1}(U)$ is a neighborhood of $x$. Hence there exists $\varphi \in D$ such that $\varphi(p) \in \nu^{-1}(U)$, so $\nu \varphi(p) \in U$. Since $\nu \in N(D)$, there exists $\psi \in D$ such that $\nu \varphi=\psi \nu$. Hence $\psi \nu(p) \in U$ and $\nu(x) \in \overline{D(\nu p)} \cap H(\infty)$.

(3) Let $U$ be a neighborhood of $x \in L_{\tau}(D)$. For any $p \in H$ there is a $\varphi \in D$ such that $\varphi(p) \in U$. Since $\{\varphi p\}$ is closed in $\bar{H}$ there is a $\psi \in D$ such that $\psi(p) \in U-\{\varnothing p\}$. Since $\psi(p)=\psi \varphi^{-1}(\varphi p)$ we have $\psi \varphi^{-1}(U) \cap U \neq \square$. Hence $D$ is not properly dicontinuous at $x$.

If $\bar{H}$ has the cone topology of the following section and if $H$ satisfies Axiom $1(\S 4)$ then $L_{\tau}(D)$ is precisely the set of points of $H(\infty)$ at which $D$ fails to act properly discontinuously (Proposition 8.5). This should be compared with the analogous result in automorphic function theory.

Points of $L_{-}(D)$ are called limit points of $D$. Points of the complement $O_{\tau}(D)=H(\infty)-L_{\tau}(D)$ are called ordinary points. Clearly $O_{\varepsilon}(D)$ is also invariant under $N(D)$ as in (2).

In the next two sections we define nontrivial admissible topologies on $\bar{H}$. In $\S 10$ we take advantage of the fact that the preceding discussion of topologies on $\bar{H}$ remains valid for any set $X$ between $H$ and $\bar{H}$, provided of course that we consider only geodesics ending in $X$ and isometries that preserve $X$.

2. The cone topology. We define a natural topology on $\bar{H}=$ $H \cup H(\infty)$ which makes $\bar{H}$ homeomorphic to the closed unit ball in $R^{n}$ and $H(\infty)$ homeomorphic to the sphere $S^{n-1}$. The notion of angle gives a natural way to measure the proximity of two points at infinity. We shall see that angle measurements also provide a notion of proximity of distant finite points to infinite points.

Let $p$ be a point of $H$ distinct from points $a, b \in \bar{H}$. The angle subtended by $a, b$ at $p$ is $\Varangle_{p}(a, b)=\Varangle\left(\gamma_{p a}^{\prime}(0), \gamma_{p b}^{\prime}(0)\right)$. If one vertex is at infinity the angle sum inequality takes the following form: let 
$x \in H(\infty), p \neq q \in H$, then $\Varangle_{p}(q, x)+\Varangle_{q}(p, x) \leqq \pi$. This follows from the usual angle sum formula and the following remark.

REMARK 2.1. If $p \in H$ and if $\alpha:[0, \infty) \rightarrow H$ is a geodesic, then the function: $[0, \infty] \rightarrow S(p)$ given by $t \rightarrow \gamma_{p, \alpha t}^{\prime}(0)$ is continuous. This is standard for $t<\infty$, and since $\gamma_{p \alpha}(\infty)$ is the unique geodesic from $p$ asymptotic to $\alpha$, the continuity at infinity follows from Proposition 1.2.

Definition 2.2. Let $v \in S(p) \subseteq H_{p}$ and let $\varepsilon$ be a number, $0<$ $\varepsilon<\pi$. Then the set $C(v, \varepsilon)=\left\{b \in \bar{H}: \chi_{p}\left(\gamma_{v}(\infty), b\right)<\varepsilon\right\}$ is called the cone of vertex $p=\mu(v)$, axis $v$ and angle $\varepsilon$.

Thus $C(v, \varepsilon)$ consists of all points, finite or infinite, whose angular difference from $v$ is less than $\varepsilon$. Note that for any $t>0$ we have $\Varangle_{p}\left(\gamma_{v} t, b\right)=\Varangle_{p}\left(\gamma_{v}(\infty), p\right)=\Varangle\left(v, \gamma_{p b}^{\prime}(0)\right)$.

Proposition 2.3. If $H$ is a Hadamard manifold, there is a unique topology $k$ on $\bar{H}$ such that

(1) $k$ has the closure property.

(2) For each $x \in H(\infty)$ the set of cones containing $x$ is a local basis for $k$ at $x$.

We call $k$ the cone topology on $\bar{H}$.

Proof. By Lemma 1.3 it suffices to prove that if $V$ and $W$ are cones containing $x \in H(\infty)$, then there exists a cone $C$ such that $x \in$ $C \subseteq V \cap W$. First we establish some properties of cones.

Lemma 2.4. Let $\alpha$ be a geodesic of $H$. If $s \leqq t$ and $\delta \leqq \varepsilon$, then $C\left(\alpha^{\prime}(t), \delta\right) \subseteq C\left(\alpha^{\prime}(s), \varepsilon\right)$.

Proof. We may suppose that $s<t$. If $b \in C\left(\alpha^{\prime}(t), \delta\right)$ then $\Varangle_{\alpha t}(b, \alpha(\infty))<\delta$ and $\Varangle_{\alpha t}(\alpha s, b)>\pi-\delta$. By the angle sum property $\Varangle_{\alpha s}(b, \alpha t)<\delta \leqq \varepsilon$. Hence $b \in C\left(\alpha^{\prime}(s), \varepsilon\right)$.

Lemma 2.5. Let $V$ be a cone with vertex $q$, let $p \in H$ and let $\beta=\gamma_{p x}$ where $x \in V \cap H(\infty)$. Then there exist numbers $T>0$ and $\delta>0$ such that $C\left(\beta^{\prime}(t), \delta\right) \subseteq V$ for $t \geqq T$.

Proof. Let $\alpha=\gamma_{q x}$ and choose $\varepsilon>0$ so that $C\left(\alpha^{\prime}(0), \varepsilon\right) \cong V$. Since $\alpha$ and $\beta$ are asymptotic $\chi_{q}(\alpha t, \beta t) \rightarrow 0$ as $t \rightarrow \infty$ by the law of cosines. Similarly $\Varangle_{\beta t}(p, q) \rightarrow 0$ as $t \rightarrow \infty$. Let $\delta=\varepsilon / 3$ and choose $T$ so large that $t \geqq T$ implies (1) $\chi_{q}(x, \beta t)<\delta$ and $(2) \chi_{\beta t}(p, q)<\delta$. We show that for $t \geqq T, C\left(\beta^{\prime}(t), \delta\right) \leqq C\left(\alpha^{\prime}(0), \varepsilon\right) \subseteq V$. Let $b \in C\left(\beta^{\prime}(t), \delta\right)$ so that (3) $\Varangle_{\beta t}(x, b)<\delta$. Then (4) $\Varangle_{\beta t}(q, x)>\pi-\delta$ follows from (2). From (3) and (4) we obtain (5) $\Varangle_{\beta t}(q, b)>\pi-2 \delta$ since $\Varangle_{\beta t}(q, b) \geqq \chi_{\beta t}(q, x)-$ 
$\Varangle_{\beta t}(x, b)$. By the angle sum relation $\Varangle_{q}(\beta t, b)+\Varangle_{\beta t}(q, b) \leqq \pi$. From (5) we obtain (6) $\Varangle_{q}(\beta t, b)<2 \delta$. Finally $\Varangle_{q}(x, b) \leqq \chi_{q}(x, \beta t)+\chi_{q}(\beta t, b)<$ $3 \delta=\varepsilon$. Therefore $b \in C\left(\alpha^{\prime}(0), \varepsilon\right) \subseteq V$.

Suppose now that $V$ and $W$ are cones that contain a point $x \in$ $H(\infty)$. Let $\beta=\gamma_{p x}$, where $p$ is the vertex of $V$. Choose $\varepsilon>0$ so that $C\left(\beta^{\prime}(0), \varepsilon\right) \subseteq V$. By Lemma 2.5 we can find $t>0$ and $\delta<\varepsilon$ such that $C\left(\beta^{\prime}(t), \delta\right) \subseteq W$. By Lemma 2.4, $C\left(\beta^{\prime}(t), \delta\right) \subseteq C\left(\beta^{\prime}(0), \varepsilon\right)$. Therefore $C\left(\beta^{\prime}(t), \delta\right) \leqq V \cap W$ and this completes the proof of Proposition 2.3.

Unless another topology is explicitly mentioned we shall always assume that $\bar{H}$ is equipped with the cone topology $k$.

Since different cones may have different vertices, it is convenient to define for each point $p$ in $H$ a collection of sets, the truncated cones at $p$, which form a basis for the cone topology. If $C(v, \varepsilon)$ is a cone with vertex $p=\mu(v)$, then for any number $r>0$ we call

$$
T(v, \varepsilon, r)=C(v, \varepsilon)-\{q \in H: d(p, q) \leqq r\}
$$

the truncated cone of vertex $p$, axis $v$, angle $\varepsilon$ and radius $r$.

Proposition 2.6. Fix a point $p$ in $H$. The set of truncated cones of vertex $p$ that contain $x \in H(\infty)$ are a local basis for the cone topology at $x$.

To prove this some preparatory lemmas are in order.

Lemma 2.7. If $V$ is a (possibly truncated) cone whose finite part $V \cap H \subseteq C(v, \varepsilon)$, then $V \subseteq C\left(v, \varepsilon^{\prime}\right)$ for any $\varepsilon^{\prime}>\varepsilon$.

Proof. Let $\alpha=\gamma_{p x}$, where $p$ is the vertex of $V$ and $x \in V \cap H(\infty)$. For sufficiently large $t>0, \alpha(t) \in C(v, \varepsilon)$ so that $\chi_{p}\left(\alpha t, \gamma_{v}(\infty)\right)<\varepsilon$. By Remark $2.1 \Varangle_{p}\left(\alpha(\infty), \gamma_{v}(\infty)\right) \leqq \varepsilon$, and therefore $x \in C\left(v, \varepsilon^{\prime}\right)$ for $\varepsilon^{\prime}>\varepsilon$.

Lemma 2.8. Let $\alpha$ be a geodesic of $H$. Given $a>0$ and $\varepsilon>0$ there exists $r>0$ and $\delta>0$ such that $T\left(\alpha^{\prime}(0), \delta, r\right) \subseteq C\left(\alpha^{\prime}(a), \varepsilon\right)$.

Proof. By the law of cosines we may choose $r>0$ so large that (1) $d(q, \alpha 0) \geqq r$ implies $\Varangle_{q}(\alpha 0, \alpha a)<\varepsilon / 3$, and (2) $d(q, \alpha r)<1$ implies $\chi_{\alpha a}(q, \alpha r)<\varepsilon / 3$, where $q$ is a point of $H$. By the continuity of the exponential map there exists a number $\delta>0$ such that if $v$ is any unit vector at $\alpha(0)$ satisfying $(3) \Varangle\left(v, \alpha^{\prime}(0)\right)<\delta$, then $d(\exp (r v), \alpha r)<$ 1. We assert that $r$ and $\delta$ are the desired numbers. Let $m$ be a finite point of $T\left(\alpha^{\prime}(0), \delta, r\right)$. Let $\beta$ be the geodesic from $\alpha(0)$ to $m$ and note that $\beta(r)$ precedes $m$. By (1) $\chi_{\beta r}(\alpha 0, \alpha \alpha)<\varepsilon / 3$, and hence $\Varangle_{\beta r}(\alpha a, m)>\pi-\varepsilon / 3$. By the angle sum property, $\Varangle_{\alpha a}(\beta r, m)<\varepsilon / 3$. Since $d(\beta r, \alpha r)<1$ by (3), we find that $\chi_{\alpha a}(\beta r, \alpha r)<\varepsilon / 3$ by (2). We conclude that $\Varangle_{\alpha a}(m, \alpha r) \leqq \chi_{\alpha a}(m, \beta r)+\Varangle_{\alpha a}(\beta r, \alpha r)<2 \varepsilon / 3$. Thus 
$m \in C\left(\alpha^{\prime}(a), 2 \varepsilon / 3\right)$, and by Lemma $2.7 T\left(\alpha^{\prime}(0), \delta, r\right) \subseteq C\left(\alpha^{\prime}(a), \varepsilon\right)$.

To prove Proposition 2.6 fix a point $p$ in $H$ and let $V$ be a cone neighborhood of $x \in H(\infty)$. Let $\beta=\gamma_{p x}$. By Lemma 2.5 we can find $t>0$ and $\delta>0$ such that $C\left(\beta^{\prime}(t), \delta\right) \leqq V$. By Lemma 2.8 we can find $r>0$ and $\delta^{\prime}>0$ such that $T\left(\beta^{\prime}(0), \delta^{\prime}, r\right) \leqq C\left(\beta^{\prime}(t), \delta\right) \leqq V$.

Proposition 2.9. The cone topology $k$ for $\bar{H}$ is admissible.

Proof. The closure property is contained in the statement of Proposition 2.3. The geodesic extension property follows immediately for Remark 2.1. If $\varphi$ is an isometry of $H$ and $C(v, \varepsilon)$ is any cone then $\varphi C(v, \varepsilon)=C\left(\varphi_{*} v, \varepsilon\right)$, so $\varphi$ is a homeomorphism of $\bar{H}$ and $k$ satisfies the isometric extension property. To prove the intensive property, let $V$ be a neighborhood of $x$ in $H(\infty)$ and let $a>0$ be given. We may assume that $V=C(v, \varepsilon)$ by the Proposition 2.3, where $v=\gamma_{p x}^{\prime}(0)$. By the law of cosines we may choose $r>0$ so large that if $d(p, m)>r, d(p, n)>r-a$ and $d(m, n)<a$, then $\Varangle_{p}(m, n)<\varepsilon / 2$. If $T=T(v, \varepsilon / 2, r)$, then $T$ is a neighborhood of $x$ such that $N_{a}(T) \cong$ $C(v, \varepsilon)$; for if $n \in N_{a}(T)$ then there exists $m \in T$ such that $d(m, n)<$ $a$. By the conditions imposed, $\Varangle_{p}(m, n)<\varepsilon / 2$ and hence $\Varangle_{p}(n, x) \leqq$ $\chi_{p}(n, m)+\chi_{p}(m, x)<\varepsilon$.

The following theorem establishes an analogy with the Poincaré ball model of the $n$-dimensional hyperbolic space.

THEOREM 2.10. If $p \in H$ let $B(p)$ be the closed unit ball in $H_{p}$ with boundary sphere $S(p)$. Let $f:[0,1] \rightarrow[0, \infty]$ be a homeomorphism. Then the function $\varphi: B(p) \rightarrow H$ such that $\varphi(v)=\exp (f\|v\| \cdot v)$ is a homeomorphism carrying $S(p)$ onto $H(\infty)$.

Proof. It is well known that $\phi$ restricted to $B(p)-S(p)$ is a one-to-one continuous map onto $H . \quad \bar{H}$ is Hausdorff, since any two distinct points in $H(\infty)$ may be separated by cones with the same vertex. Since $B(p)$ is compact if suffices to show that $\phi$ is continuous at $v \in S(p)$. For a truncated cone neighborhood $T=T(v, \varepsilon, r)$ of $\varphi(v)$, we have $\varphi^{-1}(T)=T\left(v, \varepsilon, f^{-1}(r)\right) \subseteq B(p)$, and this is clearly a neighborhood of $v$ in $B(p)$.

REMARK 2.11. For $p \in H$ the function $\varphi_{p}: S(p) \rightarrow H(\infty)$ such that $\varphi_{p}(v)=\gamma_{v}(\infty)$ is a homeomorphism. In fact this is simply the restriction of the map $\varphi$ above to $S(p)$. Since $S(p)$ is metrized by the angle function $\Varangle$, it follows that a sequence $\left\{x_{n}\right\} \subseteq H(\infty)$ converges to $x$ in $H(\infty)$ if and only if $\chi_{p}\left(x_{n}, x\right) \rightarrow 0$ as $n \rightarrow \infty$.

Corollary 2.12. If $V$ is a cone in $\bar{H}$, then $\bar{V}$ is a topological $n$-cell. 
Proof. Let $p$ be the vertex of $V$. Then $\varphi^{-1}(\bar{V})$ is a closed cone in $B(p)$ with vertex at the origin and hence is a topological $n$-cell.

We now prove the continuity of some useful functions. Let $S H \subseteq$ $T H$ be the unit and full tangent bundles of $H$, both with projection $\mu$. The map $\mu \times$ exp: $T H \rightarrow H \times H$ is a diffeomorphism and induces a metric $\delta$ on $S H$ such that $\delta(v, w)=d(\mu v, \mu w)+d(\exp v, \exp w)$.

Proposition 2.13. The function $\psi: S H \times[-\infty, \infty] \rightarrow \bar{H}$ given by $\psi(v, t)=\gamma_{v}(t)$ is continuous.

Proof. If $t$ is finite, continuity at $(v, t)$ is a standard result. It suffices to show that if $v_{n} \rightarrow v$ in $S H$ and $t_{n} \rightarrow+\infty$ as $n \rightarrow \infty$, then $\gamma_{v_{n}}\left(t_{n}\right) \rightarrow \gamma_{v}(\infty)$ as $n \rightarrow \infty$. By replacing $v_{n}$ and $v$ by their negatives, we also prove continuity at $-\infty$. Let $p=\mu(v), p_{n}=\mu\left(v_{n}\right)$. Let $w_{n}$ be the unique unit vector at $p$ such that $\gamma_{w_{n}}\left(s_{n}\right)=\gamma_{v_{n}}\left(t_{n}\right)$. Then $s_{n} \rightarrow$ $+\infty$ as $n \rightarrow \infty$. For large $n, s_{n} \geqq 1, t_{n} \geqq 1$, and $d\left(\exp v_{n}, \exp w_{n}\right) \leqq$ $d\left(p_{n}, p\right)$ by convexity. Therefore $\delta\left(v_{n}, w_{n}\right) \leqq 2 d\left(p_{n}, p\right)$, and since $p_{n} \rightarrow$ $p$, we get $w_{n} \rightarrow v$ as $n \rightarrow \infty$. The truncated cones at $p$ which contain $x$ form a local basis at $x$, and it follows that $\gamma_{v_{n}}\left(t_{n}\right)=\gamma_{w_{n}}\left(s_{n}\right) \rightarrow \gamma_{v}(\infty)$ as $n \rightarrow \infty$.

Proposition 2.14. Let $D=\{p, b) \in H \times \vec{H}: p \neq b\}$. The function $V: D \rightarrow S H$ given by $V(p, b)=\gamma_{p b}^{\prime}(0)$ is continuous.

Proof. Let $\left(p_{n}, b_{n}\right) \rightarrow(p, b)$ in $D$. Let $v_{n}=V\left(p_{n}, b_{n}\right)$ and $w_{n}=$ $V\left(p, b_{n}\right)$, which are both defined for sufficiently large $n$. If $b \in H$, then $V\left(p_{n}, b_{n}\right) \rightarrow V(p, b)$ by standard facts. Suppose that $b \in H(\infty)$, and let $T$ be a truncated cone with vertex $p$ which contains $b$. Then $b_{n} \in T$ for sufficiently large $n$, and therefore $w_{n}=V\left(p, b_{n}\right) \rightarrow V(p, b)$ as $n \rightarrow \infty$. However $\delta\left(v_{n}, w_{n}\right) \leqq 2 d\left(p_{n}, p\right)$ as in the previous argument. Since $p_{n} \rightarrow p$ as $n \rightarrow \infty$, we get $\lim _{n \rightarrow \infty} V\left(p_{n}, b_{n}\right)=\lim _{n \rightarrow \infty} V\left(p, b_{n}\right)=V(p, b)$.

Let $\psi: S H \rightarrow H(\infty)$ also denote the restriction of the function in 2.13 , so that $\psi(v)=\gamma_{v}(\infty)$. Then the functions $\mu \times \psi: S H \rightarrow H \times H(\infty)$ and $V \mid H \times H(\infty)$ are inverse homeomorphisms.

It is also easy to show that the angle function $\varnothing_{p}(a, b)$ is continuous on $\{(p, a, b) \in H \times \bar{H} \times \bar{H}: p \neq a, p \neq b\}$. In fact we have the relation $\cos \left(\Varangle_{p}(a, b)\right)=\langle V(p, a), V(p, b)\rangle$.

3. The horocycle topology. Analogous to the notion of sphere centered at a finite point $p$ of a Hadamard manifold is the notion of limit sphere at an infinite point $x \in H(\infty)$. Two points of $H$ lie on the same limit sphere at $x$ if they are "equidistant" from $x$ in a suitable sense. In the hyperbolic plane, the limit spheres are horocycles: Euclidean circles internally tangent to the unit circle.

Limit spheres have proved useful in studying the geodesics of a 
negatively curved manifold. For example, Hedlund, E. Hopf and others used them to obtain mixing and ergodic theorems for the geodesic flow on a surface of (nearly) constant negative curvature [10], [11]. The technique of Hedlund can be extended to higher dimensions [7]. Anosov has used limit spheres to prove the ergodicity of the geodesic flow on a compact manifold of negative curvature [1].

In the next section we link limit spheres to the length-minimizing properties of geodesics; in this section we show that they give rise to a natural topology on $\bar{H}$, the horocycle topology, fundamental to later work. Our basic definitions derive from Busemann [4].

If $p \in H$ and $\gamma$ is any geodesic of $H$, then the function $t \rightarrow d(p, \gamma t)-t$ is bounded below and monotone decreasing. In fact the function is strictly decreasing if $p$ does not lie on $\gamma$, for then if $s<t$ we have $d(p, \gamma t)<d(p, \gamma s)+t-s$. Thus the formula

$$
f_{\gamma}(p)=\lim _{t \rightarrow \infty} d(p, \gamma t)-t
$$

defines a real-valued function on $H$. Then [4], [7]:

(1) $f_{r}$ is uniformly continuous and convex. In fact, $\mid f_{r}(p)-$ $f_{\gamma}(q) \mid \leqq d(p, q)$.

(2) If $\beta$ is asymptotic to $\gamma$ then $f_{\gamma}(\beta s)-f_{\gamma}(\beta t)=t-s$. In particular, $f_{\gamma}$ has no minimum.

The following crucial uniformity condition is proved in [7]. The same conclusion is obtained by Busemann [4] under somewhat different hypotheses including $d(\beta, \gamma)=0$.

Proposition 3.1. If $\beta$ and $\gamma$ are asymptotic geodesics in $H$ then $f_{\beta}-f_{\gamma}$ is constant.

If $x \in H(\infty)$ and $\gamma \in x$ we call $f_{\gamma}$ a Busemann function at $x$. These functions are analogues of the radius function $q \rightarrow d(p, q)$ at a finite point $p \in H$. If $f$ is a Busemann function at $x$ and $p \in H$, then the limit sphere at $x$ through $p$ is the set $L(p, x)=\{q \in H: f(q)=f(p)\}$. The limit ball at $x$ determined by $p$ is the set $N(p, x)=\{q \in H: f(q)<$ $f(p)\}$. Because of Proposition 3.1 these definitions are independent of the choice of $f$ at $x$.

It is easy to verify that $L(p, x)$ is the topological boundary of $N(p, x)$ and that $N(p, x)$ is the convex open set $\bigcup_{t>0} N_{t}(\alpha t)$, where $\alpha$ is the geodesic such that $\alpha(0)=p$ and $\alpha(\infty)=x$. (Here $N_{t}(\alpha t)$ is the open ball with center $\alpha(t)$ and radius $t$.) The Euclidean and hyperbolic spaces represent the two extremes with respect to geometric properties of their limit spheres. In Euclidean space a limit sphere $L(p, x)$ is a hyperplane through $p$ orthogonal to the direction $x$, and the corresponding limit ball is the open halfspace composed of all geodesic rays from $p$ making angle $<\pi / 2$ with $\gamma_{p x}$. In hyperbolic space $P$ (Poincaré 
model) a limit sphere at $x$ is a Euclidean sphere in $P$ that is "tangent" to $P(\infty)$ at $x$; the only geodesic from $p$ contained in the limit ball is the geodesic $\gamma_{p x}$.

If $L$ is a limit sphere at $x$, then each geodesic $\gamma \in x$ meets $L$ at precisely one point; also $L=f_{\gamma}^{-1}(a)$ for some $a \in R$ and $f_{\gamma}(\gamma t)=-t$. Let $\eta_{L}: H \rightarrow L$ be the function that sends a point $p \in H$ to the unique point at which $\gamma_{p x}$ meets $L$. We call $\eta_{L}$ the projection on $L$.

Proposition 3.2 If $p \in H$ and $L$ is a limit sphere at $x$ then $\eta_{L}(p)$ is the unique point of $L$ nearest $p$.

Remark. A closed set $A \subseteq H$ will be called a Motzkin set if for every point $p \in H-A$ there is a unique point $\eta(p)$ in $A$ nearest $p$. Thus limit spheres are Motzkin sets. In Euclidean space a theorem due to Motzkin states that the Motzkin sets are precisely the closed convex sets. In [3] it is shown that any closed convex subset of a Hadamard manifold $H$ is a Motzkin set. The preceding proposition shows that the converse is false for arbitrary $H$. Indeed, if every Motzkin set in $H$ is convex, then $H$ is isometric to Euclidean space [4].

Proof of 3.2. Let $f$ be a Busemann function such that $L=f^{-1}(0)$. Let us suppose first that $p$ is inside $L$, that is $f(p)<0$. Let $\alpha$ be the geodesic joining $p$ to $x$, parametrized so that $\alpha(0)=\eta(p)$ where $\eta=\eta_{L}$. Then $p=\alpha(a)$ where $a=d(p, \eta(p))$. If $q \neq \eta(p)$ is any other point of $L$ then the function $t \rightarrow d(q, \alpha t)-t$ decreases strictly to zero. In particular $d(q, p)-d(\eta(p), p)=d(q, \alpha(a))-a>0$.

Suppose now that $p$ is outside $L$, that is, $f(p)>0$. Again let $\alpha$ be the geodesic joining $p$ to $x$ parametrized so that $\alpha(0)=\eta(p) \in L$. Let $q \neq \eta(p) \in L$. Then the law of cosines implies that $\Varangle_{\eta p}(q, \alpha t) \leqq$ $\pi / 2$ for any $t>0$. Hence $\Varangle_{\eta p}(p, q) \geqq \pi / 2$ and by the law of cosines $d(p, \eta p)<d(p, q)$, since $q \neq \eta(p)$.

It follows that any two limit spheres at the same point $x \in H(\infty)$ are "parallel."

CoROLlary 3.3. Let $L$ and $L^{\prime}$ be limit spheres at $x \in H(\infty)$ and let $f$ be a Busemann function at $x$. Then for any points $p \in L, p^{\prime} \in L^{\prime}$

$$
\begin{aligned}
d\left(L, L^{\prime}\right)=d\left(p, L^{\prime}\right) & =d\left(p^{\prime}, L\right)=\left|f L-f L^{\prime}\right| \\
& =\left|f(p)-f\left(p^{\prime}\right)\right| .
\end{aligned}
$$

Proof. Let $\eta$ be the projection on $L^{\prime}$. Since $\eta(p)$ lies on the geodesic $\gamma_{p x}$ it follows from (2) and 3.2 that

$$
d(p, L)=d(p, \eta p)=|f p-f \eta p|=\left|f p-f p^{\prime}\right| .
$$

Reversing the roles of $p$ and $p^{\prime}$ we obtain the desired result. 
Limit spheres induce a natural product decomposition of $H$.

Proposition 3.4. Let $f$ be a Busemann function at $x \in H(\infty)$, and let $L$ be a limit sphere at $x$. Then the function $\eta_{L} \times f: H \rightarrow L \times$ $R^{1}$ is a homeomorphism.

Proof. We may suppose that $L=f^{-1}(0)$. If $p \in H$ let $\alpha=\gamma_{p x}$ so that $\alpha^{\prime}(0)=V(p, x)$, where $V$ is the vector field of 2.14. Now $\alpha(t) \epsilon$ $L$ if and only if $f(\alpha t)=0$; hence by (2) $f(p)=f(\alpha 0)=t$. Hence $\eta_{L}(p)=$ $\exp (f(p) V(p, x))$ which shows that $\eta_{L}$ and $\eta_{L} \times f$ are continuous. The function $\xi: L \times R^{1} \rightarrow H$ given by $\xi(q, t)=\exp (-t V(q, x))$ is a continuous inverse of $\eta_{L} \times f$. If $p \in H$ then for $\alpha$ as above we have $\alpha^{\prime}(f(p))=V\left(\eta_{L}(p), x\right)$. Hence $\xi\left(\eta_{L}(p), f(p)\right)=\exp \left(-f(p) \alpha^{\prime}(f(p))=\alpha(0)=\right.$ $p$. On the other hand, if $q \in L$ and $t \in R$ then clearly $\eta_{L}(\xi(q, t))=q$ for all $t$. If $\beta$ is the geodesic from $q$ to $x$, then $\xi(p, t)=\beta(-t)$ and by $(2) f(\beta(-t))=t$.

Heretofore Busemann functions were known only to be continuous; we now show they are $C^{1}$. Although in special cases they are $C^{\infty}$, in general we do not know if they are $C^{2}$.

Proposition 3.5. Let $H$ be a Hadamard manifold, $x \in H(\infty)$, and $f$ a Busemann function at $x$. Then $f$ is $C^{1}$, and $\operatorname{grad} f=-X$ where $X(p)=V(p, x)$.

LEMMA 3.6. Let $x \in H(\infty)$ and for each integer $n \geqq 1$ let $p_{n}$ and $q_{n}$ be distinct points on a limit sphere $L_{n}$ at $x$ such that $d\left(p_{n}, q_{n}\right) \rightarrow 0$ as $n \rightarrow \infty$. If the sequence $\left\{p_{n}\right\}$ lies in a compact set then $\Varangle_{p_{n}}\left(x, q_{n}\right) \rightarrow$ $\pi / 2$ as $n \rightarrow \infty$.

Proof. If $L_{n}=f_{n}^{-1}(0)$ for a suitable Busemann function $f_{n}$ at $x$, let $P_{n}$ and $Q_{n}$ be the projections of $p_{n}$ and $q_{n}$ on $f_{n}^{-1}(1)$. Consider the angles $\theta_{n}=\Varangle_{p_{n}}\left(x, q_{n}\right), \varphi_{n}=\Varangle_{p_{n}}\left(q_{n}, Q_{n}\right)$ and $\omega_{n}=\Varangle_{p_{n}}\left(Q_{n}, P_{n}\right)$. Hence $\pi \leqq \varphi_{n}+\theta_{n}+\omega_{n}$. We will show (a) $\omega_{n} \rightarrow 0$ as $n \rightarrow \infty$, and (b) $\varphi_{n} \leqq \pi / 2$. It follows that $\lim \inf \theta_{n} \geqq \pi / 2$. But by the reasoning of Proposition $3.2 \theta_{n} \leqq \pi / 2$ for every, $n$, so the result follows.

Let $X(p)=V(p, x)$. Then by definition $P_{n}=\exp \left(-X\left(p_{n}\right)\right)$ and $Q_{n}=$ $\exp \left(-X\left(q_{n}\right)\right)$. Since $\left\{p_{n}\right\}$ and $\left\{q_{n}\right\}$ lie in a compact set and $d\left(p_{n}, q_{n}\right) \rightarrow 0$ it follows that $d\left(P_{n}, Q_{n}\right) \rightarrow 0$, by the continuity of exp and $X$. Since $d\left(p_{n}, P_{n}\right)=1$ it follows that $\omega_{n} \rightarrow 0$ as $n \rightarrow \infty$. To prove (b) we also use the law of cosines; since $q_{n}$ is the point of $L_{n}$ nearest $Q_{n}$ it follows that $d\left(q_{n}, Q_{n}\right) \leqq d\left(p_{n}, Q_{n}\right)$ and hence $\varphi_{n} \leqq \pi / 2$.

Proof of 3.5. Let $V$ be a unit vector at $P$ in $H$. We will show

$$
\left(f \circ \gamma_{v}\right)^{\prime}(0)=-\langle v, X(p)\rangle \text {. }
$$


Since $X$ is continuous, $f$ has continuous directional derivatives and is therefore a $C^{1}$ function.

Since any two Busemann functions differ by a constant we may

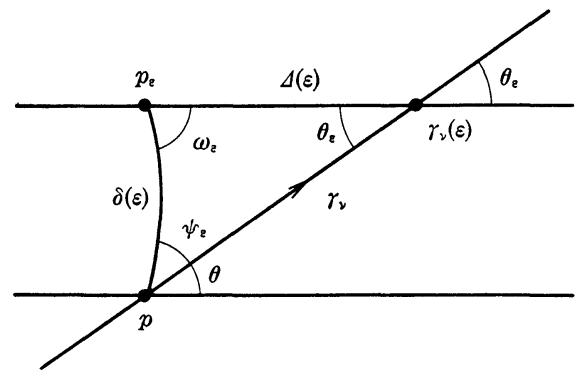

FIGURE 1

suppose that $f(p)=0$. Let $\theta=\Varangle(v,-X(p))$. It suffices to prove (*) for $\theta \leqq \pi / 2$, for if $\theta>\pi / 2$ we may replace $v$ by $-v$. For $\varepsilon>0$ let $p_{\varepsilon}$ be the projection of $\gamma_{v}(\varepsilon)$ on $L(p, x)$. Let $\Delta(\varepsilon)=d\left(p_{\varepsilon}, \gamma_{v}(\varepsilon)\right)=$ $f\left(\gamma_{v}(\varepsilon)\right)-f(p)$. Since $\cos \theta=-\langle v, X(p)\rangle$ it suffices to prove that

$$
\frac{\Delta(\varepsilon)}{\varepsilon} \rightarrow \cos \theta \quad \text { as } \quad \varepsilon \rightarrow 0 \text {. }
$$

If $\Delta(\varepsilon)=0$ for some $\varepsilon>0$ then $\theta=\pi / 2$ by the law of cosines. To prove $\left(^{* *}\right)$ in this case it suffices to prove that $f\left(\gamma_{v} t\right)=0$ for $0 \leqq$ $t \leqq \varepsilon$. If $\alpha=\gamma_{p x}$ and $0<t<\varepsilon$, then $d\left(\gamma_{v} t, \alpha s\right)-s>0$ for all $s>0$ by the law of cosines. Hence $f\left(\gamma_{v} t\right) \geqq 0$. However by the convexity of $f, f\left(\gamma_{v} t\right) \leqq \max \left\{f(p), f\left(\gamma_{v} \varepsilon\right)\right\}=0$. Thus $f\left(\gamma_{v} t\right)=0$ for $0 \leqq t \leqq \varepsilon$.

We now assume that $\Delta(\varepsilon)>0$ for all $\varepsilon>0$. We also assume that $\theta \neq 0$, otherwise the result is trivial. Hence $\delta(\varepsilon)=d\left(p, p_{\varepsilon}\right)>0$ for all $\varepsilon>0$. Consider the angles $\psi_{\varepsilon}=\chi_{p}\left(p_{\varepsilon}, \gamma_{v} \varepsilon\right), \omega_{\varepsilon}=\chi_{p_{\varepsilon}}\left(p, \gamma_{v} \varepsilon\right)$ and $\theta_{\varepsilon}=\Varangle\left(\gamma_{\nu}^{\prime}(\varepsilon),-X\left(\gamma_{v} \varepsilon\right)\right)$. We show that (a) $\omega_{\varepsilon} \rightarrow \pi / 2$ as $\varepsilon \rightarrow 0$ (b) $\theta_{\varepsilon} \rightarrow$ $\theta$ as $\varepsilon \rightarrow 0$ and (c) $\psi_{\varepsilon} \rightarrow \pi / 2-\theta$ as $\varepsilon \rightarrow 0$. Now (a) follows immediately from Lemma 3.6. Since $\cos \theta_{\varepsilon}=-\left\langle\gamma^{\prime}(\varepsilon), X\left(\gamma_{v} \varepsilon\right)\right\rangle, \cos \theta_{\varepsilon} \rightarrow \cos \theta$ as $\varepsilon \rightarrow$ 0 , and hence $\theta_{\varepsilon} \rightarrow \theta$. Let $\eta_{\varepsilon}=\Varangle_{p}\left(p_{\varepsilon},-X(p)\right)$. Again by Lemma 3.6 it follows that $\eta_{\varepsilon} \rightarrow \pi / 2$ as $\varepsilon \rightarrow 0$. Since $\psi_{\varepsilon}+\omega_{\varepsilon}+\theta_{\varepsilon} \leqq \pi$ it follows that $\eta_{\varepsilon}-\theta \leqq \psi_{\varepsilon} \leqq \pi-\left(\omega_{\varepsilon}+\theta_{\varepsilon}\right)$. By (a) and (b) and the remark above (c) follows.

By the double law of cosines $\Delta(\varepsilon) \leqq \delta(\varepsilon) \cos \omega_{\varepsilon}+\varepsilon \cos \theta_{\varepsilon}$. Since $\delta(\varepsilon) \leqq \varepsilon+\Delta(\varepsilon) \leqq 2 \varepsilon$ we obtain

$$
0 \leqq \frac{\Delta(\varepsilon)}{\varepsilon} \leqq 2 \cos \omega_{\varepsilon}+\cos \theta_{\varepsilon} .
$$

If $\theta=\pi / 2$ it follows from (a) and (b) that $\Delta(\varepsilon) / \varepsilon \rightarrow 0$ as $\varepsilon \rightarrow 0$, and henceforth we assume that $\theta<\pi / 2$. Using the double law of cosines again, we obtain 


$$
\begin{aligned}
\varepsilon & \leqq \delta(\varepsilon) \cos \psi_{\varepsilon}+\Delta(\varepsilon) \cos \theta_{\varepsilon} \\
& \leqq \cos \psi_{\varepsilon}\left(\Delta(\varepsilon) \cos \omega_{\varepsilon}+\varepsilon \cos \psi_{\varepsilon}\right)+\Delta(\varepsilon) \cos \theta_{\varepsilon},
\end{aligned}
$$

hence $\varepsilon \sin ^{2} \psi_{\varepsilon} \leqq \Delta(\varepsilon)\left(\cos \omega_{\varepsilon} \cos \psi_{\varepsilon}+\cos \theta_{\varepsilon}\right)$. Since $\theta<\pi / 2$ the term in parentheses on the right hand side is $\neq 0$ for small values of $\varepsilon$, and we obtain

$$
\frac{\sin ^{2} \psi_{\varepsilon}}{\cos \omega_{\varepsilon} \cos \psi_{\varepsilon}+\cos \theta_{\varepsilon}} \leqq \frac{\Delta(\varepsilon)}{\varepsilon}
$$

Combining (A), (B), (a), (b) and (c) we find that $\Delta(\varepsilon) / \varepsilon \rightarrow \cos \theta$ as $\varepsilon \rightarrow 0$.

We define the horocycle topology mentioned earlier. If $N$ is a limit ball at $x \in H(\infty)$, we call $N^{x}=N \cup\{x\}$ an augmented limit ball at $x$. Such sets appear as neighborhoods of $x$ in automorphic function theory.

Proposition 3.7. For a Hadamard manifold $H$ there exists a unique topology $h$ on $\bar{H}$ such that

(1) $h$ has the closure property.

(2) For each $x \in H(\infty)$ the set of augmented limit balls at $x$ is a local basis for $h$ at $x$.

Proof. Apply Lemma 1.3: $N^{x} \cap H=N$ is nonempty and open in H. $\quad N^{x} \cap N^{y} \cap H(\infty)$ is nonempty if and only if $x=y$. Since limit balls are linearly ordered by inclusion, the intersection is either $N^{x}$ or $N^{y}$.

Note that $h$ is always a $T_{1}$ topology; however it need not be Hausdorff, for example when $H=R^{n}$.

Proposition 3.8. The horocycle topology $h$ is admissible.

Proof. We must prove the isometric extension, geodesic extension and intensive properties. The isometric extension property is immediate since $\varphi N(p, x)=\varphi\left(\bigcup_{t>0} N_{t}(\alpha t)\right)=\bigcup_{t>0} N_{t}(\varphi \alpha t)=N(\varphi p, \varphi x)$, where $\alpha=\gamma_{p x}$. To prove the geodesic extension property we must show that if $N$ is any limit ball at $x$ and if $\gamma \in x$, then $\gamma(t) \in N$ for sufficiently large $t$. But $N=\left\{q \in H: f_{\gamma}(q)<a\right\}$ for some $a \in R$. Since $f_{\gamma}(\gamma t)=-t$ we have $\gamma(t) \in N$ for $t>|a|$. To prove the intensive property, let $N=\bigcup_{t<0} N_{t}(\alpha t)$ and $\varepsilon>0$ be given. Then $N^{\prime}=$ $\mathrm{U}_{t>0} N_{t}(\alpha(t+\varepsilon))$ is also a limit ball at $x=\alpha(\infty)$ and $N_{\varepsilon}\left(N^{\prime}\right)=$ $\mathrm{U}_{t>0} N_{t+\varepsilon}(\alpha(t+\varepsilon))=N$, since $N_{t}(\alpha t)$ is monotone increasing in $t$.

It follows from Proposition 1.4 that for any properly discontinuous group $D$ of isometries of $H$ there exists a horocyclic limit set $L_{h}(D)$ whose properties and relationship to the cone limit set $L(D)$ will be examined in $\S 4$ and $\S 7$.

4. Axioms. The crucial property of a Hadamard manifold is 
its convexity (or straightness according to Busemann): given points $p \neq q$ of $H$ there is a unique geodesic $\gamma_{p q}$ from $p$ to $q$. It is natural that we try to extend this property to $\bar{H}=H \cup H(\infty)$. If $p \in H$ and $x \in H(\infty)$ then we have seen that there is always a unique geodesic $\gamma_{p x}$ from $p$ to $x$. In the case of hyperbolic space the same is true for two infinite points; however this last property may fail badly. In Euclidean space, for example, the asymptote relation is parallelism, and $\alpha(\infty)=\beta(\infty)$ if and only if $\alpha(-\infty)=\beta(-\infty)$. Hence $x=\alpha(\infty)$ can be geodesically joined only to $-x=\alpha(-\infty)$. Furthermore the entire space is filled by geodesics joining $x$ to $-x$. Thus from our viewpoint Euclidean geometry is distinctly pathological.

\section{Definition 4.1. A Hadamard manifold $H$ satisfies}

Axiom 1. if for any points $x \neq y$ in $H(\infty)$ there exists at least one geodesic joining $x$ and $y$;

Axiom 2. if for any points $x \neq y$ in $H(\infty)$ there exists at most one geodesic joining $x$ to $y$.

Axiom 1 holds if the sectional curvature of $H$ satisfies $K \leqq c<0$ (see Lemma 9.10 of [3]). In fact Axiom 1 is a natural replacement for the condition $K \leqq c<0$. Many if not most of the known consequences of the curvature condition can be derived from the axiom, and as we show in the next section the axiom follows from considerably weaker curvature conditions.

Axiom 2 holds if $K<0$ and under considerably weaker conditions. For us it is distinctly subordinate to Axiom 1 and its effect is in general to sharpen results that can be derived from Axiom 1 alone. We call geodesics $\alpha$ and $\beta$ of $H$ equivalent if they have the same endpoints at infinity without regard to orientation, that is, if $\{\alpha(\infty), \alpha(-\infty)\}=\{\beta(\infty), \beta(-\infty)\}$. Thus Axiom 2 says that equivalent geodesics are the same except for parameterization.

The following property is equivalent to Axiom 1 but is often more convenient.

Definition 4.2. $H$ satisfies the Visibility Axiom if given $p \in H$ and $\varepsilon>0$ there exists a number $r=r(p, \varepsilon)$ with this property: if $\sigma:[a, b] \rightarrow H$ is a geodesic segment such that $d(p, \sigma) \geqq r$, then $\chi_{p}(\sigma a, \sigma b) \leqq \varepsilon$.

If a geodesic segment $\sigma$ is sufficiently far from $p$, then no matter how long $\sigma$ is, any two of its points subtend an arbitrarily small angle at $p$. Roughly speaking, distant geodesics look small.

REMARK 4.3. (1) The Visibility Axiom stated for geodesic seg- 
ments holds also for geodesic rays or maximal geodesics. In fact the same $r=r(p, \varepsilon)$ works, by the continuity of angles.

(2) If the Visibility Axiom holds for one point $p$ of $H$, then by Proposition 4.4 it holds at every point of $H$.

A sequence $\left\{\gamma_{n}\right\}$ of geodesics of $H$, maximal or not, converges to a geodesic $\gamma$, written $\left\{\gamma_{n}\right\} \rightarrow \gamma$, if for suitable parametrizations the tangent vectors $\left\{\gamma_{n}^{\prime}(0)\right\}$ converge in $S H$ to $\gamma^{\prime}(0)$ and the domains of $\left\{\gamma_{n}\right\}$ converge to the domain of $\gamma$. We make frequent use of the following fact: if each element of a sequence of geodesics meets a compact set of $H$, then there exists a convergent subsequence.

Proposition 4.4. The following are equivalent:

(1) $H$ satisfies the Visibility Axiom.

(2) Let $\gamma_{n}:\left[a_{n}, b_{n}\right] \rightarrow H$ be a sequence of geodesics in $H,-\infty \leqq$ $a_{n}<b_{n} \leqq \infty$. If $\gamma_{n}\left(a_{n}\right) \rightarrow x$ and $\gamma_{n}\left(b_{n}\right) \rightarrow y$ as $n \rightarrow \infty$, where $x$ and $y$ are distinct points of $H(\infty)$, then every $\gamma_{n}$ meets some compact set $K$ of $H$. Hence some subsequence of $\left\{\gamma_{n}\right\}$ converges to a geodesic joining $x$ to $y$.

Proof. Suppose Visibility holds at a point $p \in H$. Then with the notation of (2) the sequence $\left\{\chi_{p}\left(\gamma_{n} a_{n}, \gamma_{n} b_{n}\right)\right\}$ converges to $\Varangle_{p}(x, y)$ by the continuity of angles. Since $x \neq y, \Varangle_{p}(x, y)>0$; and Remark 4.3 (1) implies that there exists a number $r>0$ such that $d\left(p, \gamma_{n}\right) \leqq r$ for every integer $n$. Hence $B_{r}(p)$ meets every $\gamma_{n}$. Choosing a subsequence if necessary and parametrizing suitably, we find that $\left\{\gamma_{n}^{\prime}(0)\right\}$ converges to a vector $v \in S(q)$. Hence $\gamma_{n} \rightarrow \gamma_{v}$. By Proposition 2.14 the sequence $\left\{\gamma_{n}^{\prime}(0)\right\}=\left\{V\left(\gamma_{n} 0, \gamma_{n} b_{n}\right)\right\}$ converges to $V(q, y)$ and the sequence $\left.\left\{-\gamma_{n}^{\prime}(0)\right\}=\left\{V \gamma_{n} 0, \gamma_{n} a_{n}\right)\right\}$ converges to $V(q, x)$. Hence $\gamma_{v}$ joins $x$ to $y$.

To prove the converse, suppose that Visibility fails. Then for some point $p \in H$ and some $\varepsilon>0$ there exists a sequence $\left\{\gamma_{n}\right\}$ of geodesics of $H$ such that $d\left(p, \gamma_{n}\right) \rightarrow \infty$ as $n \rightarrow \infty$ and $\chi_{p}\left(\gamma_{n} a_{n}, \gamma_{n} b_{n}\right) \geqq$ $\varepsilon$ for every $n$. Choosing a subsequence if necessary, $\left\{\gamma_{n}\left(a_{n}\right)\right\} \rightarrow x \in$ $H(\infty),\left\{\gamma_{n}\left(b_{n}\right)\right\} \rightarrow y \in H(\infty)$, and $\chi_{p}(x, y) \geqq \varepsilon>0$. Therefore $x \neq y$. But by (2) every $\gamma_{n}$ meets some compact set which contradicts our hypothesis that $d\left(p, \gamma_{n}\right) \rightarrow \infty$ as $n \rightarrow \infty$.

It follows that the Visibility Axiom implies Axiom 1. The converse is proved in [13], thus we use the terms Axiom 1 and Visibility interchangeably. If $M=H / D$ is a complete manifold with curvature $K \leqq 0$ such that $H$ satisfies Axiom 1, we call $M$ a Visibility manifold.

CoRollary 4.5. If $H$ satisfies Axiom 1 then for any two points $x \neq y$ of $H(\infty)$ there exists a compact set $K$ of $H$ such that every geodesic joining $x$ to $y$ meets $K$. 
The Visibility property is uniform on compact subsets:

COROLlary 4.6. Let $H$ satisfy the Visibility Axiom. Let $K, a$ compact subset of $H$, and $\varepsilon>0$ be given. Then there exists a number $r=r(K, \varepsilon)$ such that if $\sigma:[a, b] \rightarrow H$ is a geodesic segment satisfying the condition $d(\sigma, K) \geqq r$ then $\Varangle_{p}(\sigma a, \sigma b) \leqq \varepsilon$ for every point $p \in K$.

Proof. Assume the proposition is false. Then there exists a sequence $\left\{p_{n}\right\} \subseteq K$ and a sequence $\left\{\sigma_{n}\right\}$ of geodesic segments, $\sigma_{n}:\left[a_{n}, b_{n}\right] \rightarrow$ $H$, such that $d\left(\sigma_{n}, K\right) \rightarrow \infty$ as $n \rightarrow \infty$ but $\chi_{p_{n}}\left(\sigma_{n} a_{n}, \sigma_{n} b_{n}\right) \geqq \varepsilon>0$ for some $\varepsilon>0$ and every integer $n$. Choosing subsequences if necessary, $\left\{p_{n}\right\} \rightarrow p \in K,\left\{\sigma_{n} a_{n}\right\} \rightarrow x \in H(\infty)$ and $\left\{\sigma_{n} b_{n}\right\} \rightarrow y \in H(\infty)$. By continuity, $\chi_{p}(x, y) \geqq \varepsilon>0$ and hence $x \neq y$. By Proposition 4.4 every $\sigma_{n}$ meets some compact subset of $H$, contradicting the hypothesis that $d\left(\sigma_{n}, K\right) \rightarrow$ $\infty$ as $n \rightarrow \infty$.

We derive further consequences of Axiom 1. If $p \notin A \subseteq \bar{H}$ the angle $\Varangle_{p}(A)$ subtended by $A$ at $p$ is $\sup \left\{\Varangle_{p}(a, b): a, b \in A\right\}$.

Proposition 4.7. Let $H$ satisfy Axiom 1. Let $\left\{p_{n}\right\}$ be a sequence in $H$ that converges to $x \in H(\infty)$. Let $W$ be any neighborhood of $x$ in $\bar{H}$. Then $\Varangle_{p_{n}}(\bar{H}-W) \rightarrow 0$ as $n \rightarrow \infty$.

Proof. We may suppose that $W$ is a cone $C\left(\alpha^{\prime}(0), \varepsilon\right)$ where $\alpha=$ $\gamma_{p x}$. We must show that for any sequences $\left\{a_{n}\right\}$ and $\left\{b_{n}\right\} \subseteq \bar{H}-W$ the angle $\Varangle_{p_{n}}\left(a_{n}, b_{n}\right) \rightarrow 0$ as $n \rightarrow \infty$. It suffices to show that for any sequence $\left\{b_{n}\right\} \subseteq \bar{H}-W$ we have $\chi_{p_{n}}\left(p, b_{n}\right) \rightarrow 0$ as $n \rightarrow \infty$. For sufficiently large $n, \Varangle_{p}\left(p_{n}, b_{n}\right) \geqq \varepsilon / 2$ since $p_{n} \rightarrow x$ and $b_{n} \notin C\left(\alpha^{\prime}(0), \varepsilon\right)$. Let $\sigma_{n}$ be the geodesic from $p_{n}$ to $b_{n}$. By Visibility, $d\left(p, \sigma_{n}\right) \leqq r$ for every $n$ and some $r>0$. Let $q_{n}$ be a point on $\sigma_{n}$ such that $d\left(p, q_{n}\right) \leqq r$. By the law of cosines, $\Varangle_{p_{n}}\left(p, q_{n}\right) \rightarrow 0$ as $n \rightarrow \infty$. But for large $n, p_{n} \neq$ $q_{n}$ and hence $\Varangle_{p_{n}}\left(p, b_{n}\right)=\Varangle_{p_{n}}\left(p, q_{n}\right)$. The result follows.

In Euclidean space a limit ball is a half space, so the horocycle topology is rather small and bears little relation to the cone topology. The situation is much different if $H$ satisfies Axiom 1.

Proposition 4.8. Let $H$ satisfy Axiom 1. Let $W$ be a cone containing a point $x \in H(\infty)$. Then $W$ contains some limit ball $N(p, x)$. Thus the horocycle topology is larger than the cone topology.

Proof. Let $W$ be a cone $C\left(\alpha^{\prime}(0), \varepsilon\right)$, where $\alpha=\gamma_{p x}$. By Lemma 2.5 it suffices to prove the result for $W$ of this form. Let $r=r(p, \varepsilon / 2)$ be the number in Definition 4.2. Then the limit ball $N(\alpha(r), x)$ is contained in $W$. If $q \in N(\alpha(r), x)$ then there exists $t>0$ such that $q \in N_{t}(\alpha(r+t))$. The geodesic segment $\sigma$ from $\alpha(r+t)$ to $q$ lies in 
$N_{t}(\alpha(r+t))$, hence $d(p, \sigma) \geqq r . \quad$ Therefore $\Varangle_{p}(x, q)=\Varangle_{p}(\alpha(r+t), q) \leqq$ $\varepsilon / 2<\varepsilon$. Hence $q \in W$.

The following result is immediate.

COROLlaRY 4.9. Let $D$ be a properly discontinuous group of isometries of $H$. Let $L_{h}(D)$ and $L(D)$ be the horocycle and cone limit sets respectively. Then $L_{h}(D) \subseteq L(D)$ if $H$ satisfies Axiom 1 .

The corollary fails seriously in Euclidean space. For example, let $D$ be the cyclic group of isometries of $R^{2}$ generated by the translation $T: T(u, v)=(u+1, v)$. Then $L(D)$ consists of two points, the endpoints of the geodesic represented by the $x$-axis. $L_{h}(D)$, however, contains every point of $R^{2}(\infty)$ except the endpoints of the $y$-axis.

If $H$ satisfies both Axiom 1 and Axiom 2 then there is a unique geodesic $\gamma_{a b}$ joining any two distinct points $a, b \in \bar{H}$. Furthermore $\bar{H}$ is continuously convex: if $\left\{a_{n}\right\} \rightarrow a,\left\{b_{n}\right\} \rightarrow b$ and $\gamma_{n}=\gamma_{a_{n} b_{n}}$, then the sequence $\left\{\gamma_{n}\right\}$ converges to $\gamma_{a b}$. This follows from Proposition 4.4.

In the remainder of this section we discuss some geometric properties of $H(\infty)$ related to Axioms 1 and 2. These "boundedness" conditions on $H(\infty)$ strongly influence the behavior of the asymptote classes in any quotient manifold $H / D$.

A point $x \in H(\infty)$ is special if for any $\gamma \in x$ there exists a compact set $K \subseteq H$ such that for arbitrarily large positive values of $t, \gamma$ meets $I(H) K=\{\varphi(k): k \in K, \varphi$ an isometry of $H\}$. By definition of the asymptote relation it suffices to verify this property for a single geodesic $\gamma \in x$. If $H$ is homogeneous or has a compact quotient $H / D$, then every point of $H(\infty)$ is special.

If $a, b$ are distinct points of $H(\infty)$ let $a \wedge b$ be the set of all geodesics joining $a$ to $b$. The pair $(a, b)$ is bounded if there exists a compact set $K=K(a, b)$ such that any geodesic in $a \wedge b$ meets $K$. (If $a \wedge b$ is empty, the pair $(a, b)$ will also be called bounded.) $H$ is geodesically bounded if every pair $(a, b)$ with $a \neq b$ in $H(\infty) \times H(\infty)$ is bounded. If $H$ satisfies either Axiom 1 or Axiom 2, then $H$ is geodesically bounded.

A point $x \in H(\infty)$ is bounded if there exists $C>0$ such that $d(\gamma, \sigma) \leqq C$ for any two geodesics $\gamma, \sigma \in x$. The convexity of the function $t \rightarrow d(\gamma t, \sigma)$ implies that if every point of $H(\infty)$ is bounded, then $H$ is geodesically bounded. We now prove a partial converse.

Proposition 4.10. Let $H$ be geodesically bounded and let $x \in H(\infty)$ be special. Then $x$ is bounded.

Lemma 4.11. Let $\alpha$ and $\beta$ be geodesics of $H$ such that $\alpha(\infty)=$ 
$\beta(\infty)=x$. If $\left\{p_{n}\right\}$ is any sequence in $H$ converging to $x$ then $\chi_{p_{n}}(\alpha(-\infty), \beta(-\infty)) \rightarrow 0$ as $n \rightarrow \infty$.

Proof. Let $q$ and $q^{*}$ be points in $H$ that lie on $\alpha$ and $\beta$ respectively. By the angle sum property, $\Varangle_{p_{n}}\left(q^{*}, \beta(-\infty)\right) \leqq \Varangle_{q^{*}}\left(p_{n}, x\right) \rightarrow 0$ as $n \rightarrow \infty$. The same argument shows that $\chi_{p_{n}}(q, \alpha(-\infty)) \rightarrow 0$ as $n \rightarrow \infty$. Since $\Varangle_{p_{n}}\left(q, q^{*}\right) \rightarrow 0$ as $n \rightarrow \infty$, the law of cosines implies that $\chi_{p_{n}}(\alpha(-\infty), \beta(-\infty)) \leqq \chi_{p_{n}}(\alpha(-\infty), q)+\chi_{p_{n}}\left(q, q^{*}\right)+\Varangle_{p_{n}}\left(q^{*}, \beta(-\infty)\right) \rightarrow$ 0 as $n \rightarrow \infty$.

Proof of 4.10. Let $x \in H(\infty)$ be special and let $\alpha \in x$. Then there exists a bounded sequence $\left\{p_{n}\right\} \subseteq H$, a sequence $\left\{t_{n}\right\} \subseteq R$ and a sequence $\left\{\varphi_{n}\right\} \subseteq I(H)$ such that $\varphi_{n}\left(p_{n}\right)=\alpha\left(t_{n}\right)$, where $t_{n} \rightarrow+\infty$ as $n \rightarrow \infty$. By choosing subsequences if necessary, $\left\{p_{n}\right\}$ converges to a point $p$ and $\left\{\left(\varphi_{n}^{-1} \circ \alpha\right\}^{\prime}\left(t_{n}\right)\right\}$ converges to a unit vector $v$ at $p$. By continuity $\varphi_{n}^{-1}(x) \rightarrow$ $a=\gamma_{v}(\infty)$ and $\varphi_{n}^{-1} \alpha(-\infty) \rightarrow b=\gamma_{v}(-\infty)$ as $n \rightarrow \infty$. Let $\beta \in x$ be arbitrary. We show that some subsequence of $\left\{\varphi_{n}^{-1} \circ \beta\right\}$ converges to a geodesic $\beta_{*}$ in $a \wedge b$. Since $d\left(p, \varphi_{n}^{-1} \circ \beta\right)=d\left(\varphi_{n} p, \beta\right)$ is bounded, some subsequence $\left\{\varphi_{n_{k}}^{-1} \circ \beta\right\}$ converges to a geodesic $\beta^{*}$. By Lemma 4.11, $\chi_{p}\left(\varphi_{n_{k}}^{-1} \beta(-\infty), \varphi_{n_{k}}^{-1} \alpha(-\infty)\right)=\chi_{\varphi_{n_{k}} p}(\beta(-\infty), \alpha(-\infty)) \rightarrow 0$ as $k \rightarrow \infty$. Therefore $\varphi_{n_{k}}^{-1} \beta(-\infty) \rightarrow b, \varphi_{n_{k}}^{-1} \beta(\infty) \rightarrow a$ as $k \rightarrow \infty$, and $\beta^{*}$ lies in $a \wedge b$.

If $\beta, \gamma$ are any two geodesics in $x$, let $\left\{\varphi_{n_{k}}\right\}$ be a subsequence such that $\left\{\varphi_{n_{k}}^{-1} \circ \beta\right\}$ and $\left\{\varphi_{n_{k}}^{-1} \circ \gamma\right\}$ converge to geodesics $\beta^{*}$ and $\gamma^{*}$ in $a \wedge b$. Then $d(\gamma, \beta)=d\left(\varphi_{n_{k}}^{-1} \gamma, \varphi_{n_{k}}^{-1} \beta\right) \leqq d\left(\gamma^{*}, \beta^{*}\right)$ by continuity. If $R$ is any bound for the distance between any two geodesics in $a \wedge b$, then $d(\gamma, \beta) \leqq R$ for $\gamma, \beta \in x$. Hence $x$ is bounded.

We say that $x \in H(\infty)$ is a zero point if $d(\gamma, \sigma)=0$ for any two geodesics $\gamma, \sigma \in x . H$ satisfies the Zero Axiom if every $x \in H(\infty)$ is a zero point.

Remark 4.12. The Zero Axiom implies Axiom 2 in a Hadamard manifold $H$.

Proof. Suppose that geodesics $\gamma$ and $\sigma$ have the same endpoints in $H(\infty)$. Since $t \rightarrow d(\gamma t, \sigma)$ is bounded and convex it is constant. But since $d(\gamma t, \sigma) \rightarrow d(\gamma, \sigma)=0$ as $t \rightarrow \infty$, it follows that $\gamma=\sigma$.

The following result is contained in the last paragraph of the proof of 4.10 .

Proposition 4.13. If $x \in H(\infty)$ is special and $H$ satisfies Axiom 2 then $x$ is a zero point.

Corollary 4.14. If every point of $H(\infty)$ is special, then Axiom 2 and the Zero Axiom are equivalent. 
5. Curvature conditions. We find conditions on the sectional curvature $K$ of a Hadamard manifold $H$ that imply that $H$ satisfies, separately, Axiom 1 and Axiom 2. We also show that these axioms are independent.

By a flat strip in $H$ we mean a totally geodesic isometric imbedding $\boldsymbol{x}: R \times[a, b] \rightarrow H$. (Here it is understood that $\boldsymbol{x}$ is $C^{\infty}$ extendable to a neighborhood of $R \times[a, b]$ in the Euclidean plane, although of course the required properties need hold only on $R \times$ $[a, b])$. In paraticular, each longitudinal curve $\alpha_{u}(t)=\boldsymbol{x}(t, u)$ of $\boldsymbol{x}$ is a geodesic of $H$ and the flat strip is uniquely determined by its boundary curves $\alpha_{a}$ and $\alpha_{b}$. Note that all longitudinal geodesics of $\boldsymbol{x}$ are equivalent, that is, they have the same endpoints in $H(\infty)$. Conversely:

Proposition 5.1. If distinct geodesics $\alpha$ and $\beta$ of $H$ are equivalent then they are the boundary curves of a flat strip in $H$.

Proof. The equivalence of $\alpha$ and $\beta$ means that, reversing orientation if necessary, $\alpha$ and $\beta$ are asymptotic and so are the reverse curves $\alpha^{-}$and $\beta^{-}$. Hence for some $C>0, d(\alpha t, \beta t) \leqq C$ for all $t \in R$. Furthermore we may suppose that $\beta$ is parametrized so that $\beta(0)$ is the foot of $\alpha(0)$ on $\beta$. If $c=d(\alpha, \beta)$, let $x: R \times[0, c] \rightarrow H$ be the function $\boldsymbol{x}(t, u)=\sigma_{t}(u)$ where $\sigma_{t}:[0, c] \rightarrow H$ is the constant-speed geodesic segment from $\alpha t$ to $\beta t$. We show that $\boldsymbol{x}$ is a flat strip where each $\sigma_{t}$ has unit speed.

The functions $f(t)=d(\alpha t, \beta t)$ and $g(t)=d(\alpha t, \beta)$ are bounded and convex on $R$, hence constant. Thus $f(t)=f(0)=g(0)=d(\alpha, \beta)$; that is $d(\alpha t, \beta t)=L\left(\sigma_{t}\right)=c$ for all $t$. It follows that each transversal $\sigma_{t}$ is orthogonal to both $\alpha$ and $\beta$, and each $\sigma_{t}$ has unit speed.

Consider the vector fields $\boldsymbol{x}_{t}=\boldsymbol{x}_{*}(\partial / \partial t)$ and $\boldsymbol{x}_{u}=\boldsymbol{x}_{*}(\partial / \partial u)$ on the mapping $\boldsymbol{x}$. On $\alpha$ or $\beta$ these are orthogonal unit vectors, and, since $\boldsymbol{x}_{t}$ is a Jacobi field along each transversal, $\left\langle\boldsymbol{x}_{t}, \boldsymbol{x}_{u}\right\rangle=0$. Since $u \rightarrow$ $\left\|\boldsymbol{x}_{t}(u)\right\|^{2}$ is a convex function it follows that $\left\|\boldsymbol{x}_{t}\right\| \leqq 1$. Now fix $a<b$ and let $h(u)=d\left(\sigma_{a} u, \sigma_{b} u\right)$. By orthogonality $h^{\prime}(0) \geqq 0$. Since $h$ is convex and $h(0)=h(c)=b-a$ we see that $h$ is constant. Thus for arbitrary $u \in[0, c]$ the curve segment $[a, b] \rightarrow H$ given by $t \rightarrow \boldsymbol{x}(t, u)$ has speed $\left\|\boldsymbol{x}_{t}\right\| \leqq 1$, but joins points at distance $b-a$. Consequently these curves are unit speed geodesics of $H$. Since $\boldsymbol{x}_{t}, \boldsymbol{x}_{u}$ is orthonormal, $\boldsymbol{x}$ is an isometric immersion. Because $H$ has sectional curvature $K \leqq 0$ and the parameter curves of $\boldsymbol{x}$ are geodesics it follows from the Gauss equation that $\boldsymbol{x}$ is totally geodesic. Since $H$ is Hadamard, $\boldsymbol{x}$ is necessarily an imbedding.

On any plane tangent to a flat strip the sectional curvature of $H$ is zero; hence 
Corollary 5.2. If $K<0$ then $H$ satisfies Axiom 2.

In many classical arguments $K<0$ can be replaced by Axiom 2. On the other hand at least in dimension two it is not hard to construct "nearly flat" Hadamard manifolds that satisfy Axiom 2. It suffices to arrange for every geodesic eventually to pass through a point of negative curvature.

We now consider a more quantitative way to measure globally the curvature of a Hadamard manifold. Axiom 1 (like all the other axioms in the preceding section) holds for $K \leqq c<0$ but not for $K=0$. Thus it is natural to look for ways to weaken the stringent condition $K \leqq c<0$ while still maintaining Axiom 1. Roughly speaking, our scheme is to allow $K$ to approach zero on tangent planes going to infinity in $H$ and to measure the rate of approach.

Let $p \in H$. For $v \in S(p)$ and $t \geqq 0$ let $k_{v}(t)$ be the minimum of $|K(\pi)|$ for all planes $\pi$ containing $\gamma_{v}^{\prime}(t)$. We say that $H$ has curvature order at most $a \in R$ at $p$ provided that $\int_{1}^{\infty} k_{v}(t) t^{a-1} d t=\infty$ for all $v \in S(p)$.

If a smallest such number exists it is called the curvature order $\omega$ of $H$ at $p$. Intuitively the larger $\omega$ is the flatter $H$ is. For example, if $K \leqq c<0$ then $H$ has curvature order $\leqq 0$ at every point. Evidently Euclidean space must be given curvature order $+\infty$, since $k_{v} \equiv 0$ for all $v$. We will consider only $\omega \leqq 2$ (however see Proposition 5.11).

\section{EXAMPLE 5.3. A Hadamard manifold of curvature order 2.}

Let $H$ be the plane $R^{2}$ with Riemannian structure given classically by $E=1, F=0 \quad G(u, v)=\left(1+u^{2}\right)^{2}$. Then $H$ has curvature $K=$ $-2 /\left(1+u^{2}\right)$. We assert that $H$ has curvature order 2 at the origin (and in fact at any point).

Let $\alpha$ be a unit speed geodesic from the origin and let $k(t)=k_{\alpha^{\prime}(0)}(t)=$ $2 /\left(1+u(\alpha t)^{2}\right)$. By a well known result of Clairaut the function $g(\alpha) \sin \phi$ is constant, where $g(u)=1+u^{2}$ and $\phi$ is the usual slope angle of $\alpha$. In the special case where $\alpha$ parametrizes the $v$-axis we have $\int_{1}^{\infty} k(t) t d t=\infty$; otherwise $\varphi(t) \rightarrow 0$ as $t \rightarrow \infty$. Since $E=1$ it follows that $u(\alpha t) / t \rightarrow 1$ as $t \rightarrow \infty$, and hence for any number $a$ the integrals $\int_{1}^{\infty} k(t) t^{a-1} d t$ and $\int_{1}^{\infty} 2 t^{a-1} /\left(1+t^{2}\right)$ either both converge or both diverge. Hence $\int_{1}^{\infty} k(t) t d t$ diverges and $\int_{1}^{\infty} k(t) t^{a-1}$ converges for $a<2$. This shows that $H$ has curvature order 2 at the origin. In a similar way one can construct examples of any curvature order.

A simple sufficient condition for curvature order is obtained as 
follows. Fix $p \in H$ and for each $t \geqq 0$ let $\mu(t)=\min \left\{k_{v}(s): v \in S(p)\right.$ and $0 \leqq s \leqq t\}$. Thus $\mu(t)$ is the minimum of the absolute value of sectional curvature on radial tangent planes at points of $B_{t}(p)$.

REMARK 5.4. If $a>0$ is a number such that $t^{a} \mu(t) \rightarrow \infty$ as $t \rightarrow$ $\infty$, then $H$ has curvature order $\leqq a$ at $p$. In fact if $v \in S(p)$ and $0 \leqq s \leqq t$ then $k_{v}(s) \geqq \mu(t)$, and therefore $\int_{1}^{t} k_{v}(s) s^{a-1} d s \geqq \mu(t)\left(t^{a}-1\right)_{l}^{\prime} a$.

Our aim now is to show that if $H$ has curvature order $\leqq 2$ at one point $p$ then Axiom 1 holds. To do so we shall apply the GaussBonnet theorem to the "surface" consisting of the union of all geodesic segments from $p$ to a geodesic segment $\sigma:[0, b] \rightarrow H$ that does not contain $p$. Actually there may be no tangent plane at $p$, but the Gauss-Bonnet theorem remains valid if the angle $\chi_{p}(\sigma 0, \sigma b)$ is replaced by the generally larger number $\Theta(p, \sigma)$ defined below.

We may assume that $p$ does not lie on the geodesic extension $\tilde{\sigma}: R \rightarrow H$. For each $u \in[0, b]$ let $r(u)=d(p, \sigma u)$ and let $\rho_{u}:[0, r(u)] \rightarrow$ $H$ be the geodesic from $p$ to $\sigma(u)$. Let $D=\{(t, u): 0 \leqq t \leqq r(u), 0 \leqq$ $u \leqq b\}$. We call the image of $\boldsymbol{x}: D \rightarrow H$ given by $(t, u) \rightarrow \rho_{u}(t)$ the radial triangle $\Sigma$ from $p$ to $\sigma$. Let $\pi \sigma:[0, b] \rightarrow S(p)$ be the curve in the unit sphere $S(p)$ defined by $(\pi \sigma)(u)=\rho_{u}^{\prime}(0)$. With the usual Riemannian structure on $S(p)$ we call the length $L(\pi \sigma)$ of $\pi \sigma$ the curvilinear angle $\Theta(p, \sigma)$ subtended by $\sigma$ at $p$. Since $\Varangle_{p}(v, w)$ is the Riemannian distance in $S(p)$ from $v$ to $w$, for any two vectors $v$ and $w$ it follows that $\Varangle_{p}(\sigma 0, \sigma b) \leqq \Theta(p, \sigma)$. Let $\omega_{0}=\Varangle_{\sigma 0}(p, \sigma b)$ and $\omega_{b}=$ $\Varangle_{o b}(p, \sigma 0)$.

Proposition 5.5. A radial triangle $\Sigma$ in a Hadamard manifold $H$ is a surface except possibly at $p$, and if $G^{*}$ denotes its Gaussian curvature then

$$
\iint_{\Sigma} G^{*} d A=\Theta(p, \sigma)+\omega_{0}+\omega_{b}-\pi
$$

To prove this we need two somewhat technical lemmas. Subscripts $t$ and $u$ on $\boldsymbol{x}$ denote covariant differentiation, relative to the Riemannian structure of $H$, along the parameter curves of $\boldsymbol{x}$. Note that by the Gauss Lemma $\left\langle\boldsymbol{x}_{t}, \boldsymbol{x}_{u}\right\rangle=0$.

Lemma 5.6. For fixed $u \in[0, b]$ let $u^{*} \in H_{p}$ be the vector canonically corresponding to $(\pi \sigma)^{\prime}(u)$. Then

(1) $\boldsymbol{x}_{u t}(0, u)=u^{*}$

(2) $u^{*} \neq 0$

(3) $\partial / \partial t\left\|\boldsymbol{x}_{u}(t, u)\right\| \downarrow\left\|u^{*}\right\|$ as $t \rightarrow 0$

(4) $\left\|\boldsymbol{x}_{u}(t, u)\right\| \geqq t\left\|u_{*}\right\|$ for $t \geqq 0$. 
Proof. (1) By the first structural equation $\boldsymbol{x}_{u t}=\boldsymbol{x}_{t u}$. But $\boldsymbol{x}_{t u}(0, u)$ is just the Euclidean derivative of the function $u \rightarrow \boldsymbol{x}_{t}(0, u)=$ $\rho_{u}^{\prime}(0)=(\pi \sigma)(u) \in H_{p}$.

(2) For all $u \in[0, b]$ we have $\sigma(u)=\boldsymbol{x}(r(u), u)$. Hence $\sigma^{\prime}(\mu)=$ $r^{\prime}(u) \boldsymbol{x}_{t}(r(u), u)+\boldsymbol{x}_{u}(r(u), u)$. Since $\boldsymbol{x}_{t}(r(u), u)=\rho_{u}^{\prime}(r(u))$, and $\rho_{u}$ and $\sigma$ cross transversally $\left(p\right.$ is not on $\widetilde{\sigma}$ ) we find that $\boldsymbol{x}_{u}(r(u), u) \neq 0$. Let $Y(t)=\boldsymbol{x}_{u}(t, u) . \quad Y$ is a Jacobi vector field on $\rho_{u}$ such that $Y(0)=$ 0 and $Y$ is orthogonal to $\rho_{n}$. If $Y^{\prime}(0)=\boldsymbol{x}_{u t}(0, u)$ were zero then we would have $Y \equiv 0$ which contradicts the fact that $Y(r(u))=\boldsymbol{x}_{u}(r(u), u) \neq$ 0 .

(3) From [2] we obtain $\lim _{t \rightarrow 0^{+}}\|Y\|^{\prime}(t)=\left\|Y^{\prime}(0)\right\|=\left\|u^{*}\right\|$, by (1). Since $K \leqq 0$ and $Y$ is a Jacobi field, $\|Y\|^{\prime \prime}(t) \geqq 0$ for $t>0$. Hence the limit is monotone decreasing.

(4) Let $g(t)=\left\|\boldsymbol{x}_{u}(t, u)\right\|-t\left\|u^{*}\right\|$. From the results above it follows that $g(0)=0, g^{\prime}(t) \geqq 0$ for $t>0$ and $g^{\prime \prime}(t) \geqq 0$ for $t>0$. Hence $g(t) \geqq 0$ for $t \geqq 0$.

The function $x: D \rightarrow H$ is a $C^{\infty}$ imbedding except possibly at $\boldsymbol{x}^{-1}(p)$. In fact $E=\left\|\boldsymbol{x}_{t}\right\|^{2}=1, F=\left\langle\boldsymbol{x}_{t}, \boldsymbol{x}_{u}\right\rangle=0$ and $G=\left\|\boldsymbol{x}_{u}\right\|^{2}>0$ for $t>0$. Thus $\boldsymbol{x}$ is an immersion and by Hadamard properties it is necessarily an imbedding.

Lemma 5.7. Let $\Gamma(t)$ be the total geodesic curvature of the parameter curve $\tau_{t}:[0, b] \rightarrow H$ given by $u \rightarrow \boldsymbol{x}(t, u)$. Then $\lim _{t \rightarrow 0} \Gamma(t)=$ $\Theta(p, \sigma)$.

Proof. Because $E=1$ and $F=0$ for $\boldsymbol{x}$, if the radial suface $\Sigma=\boldsymbol{x}(D)$ is oriented by $\left(\boldsymbol{x}_{t}, \boldsymbol{x}_{u}\right)$ we have

$$
\Gamma(t)=\int_{\tau_{t}} \kappa_{g} d s=-\int_{0}^{b} \frac{\left\langle\boldsymbol{x}_{u u}, \boldsymbol{x}_{t}\right\rangle}{\left\|\boldsymbol{x}_{u}\right\|}(t, u) d u
$$

for $t>0$. A priori $\boldsymbol{x}_{u u}$ is computed using Riemannian structure of $\Sigma$ as a submanifold of $H$, but we may assume that it derives from the structure of $H$ itself, since the difference is normal to $\Sigma$ hence orthogonal to $\boldsymbol{x}_{t}$. Now $-\left\langle\boldsymbol{x}_{u u}, \boldsymbol{x}_{t}\right\rangle=-\partial / \partial u\left\langle\boldsymbol{x}_{u}, \boldsymbol{x}_{t}\right\rangle+\left\langle\boldsymbol{x}_{u}, \boldsymbol{x}_{t u}\right\rangle=\left\langle\boldsymbol{x}_{u}, \boldsymbol{x}_{t u}\right\rangle$. Hence

$$
\begin{aligned}
\Gamma(t) & =\int_{0}^{b} \frac{\left\langle\boldsymbol{x}_{u t}, \boldsymbol{x}_{u}\right\rangle}{\left\|\boldsymbol{x}_{u}\right\|}(t, u, d u \\
& =\int_{0}^{b} \frac{\partial}{\partial t}\left\|\boldsymbol{x}_{u}\right\|(t, v) d u \text { for } t>0 .
\end{aligned}
$$

By Lemma 5.6 (3) the integrand is monotone decreasing to $\left\|u^{*}\right\|=$ $\left\|\pi \sigma^{\prime}(u)\right\|$, so the result follows.

Proof of Proposition 5.5. For $0<t<d(p, \sigma)$ let $\Sigma_{t}=\{q \in \Sigma: d(p, q) \geqq$ 
$t$. Using the preceding lemmas the result is obtained by taking limits as $t \rightarrow 0$ in the Gauss-Bonnet formula for $\Sigma_{t}$.

LEMmA 5.8. Let $H$ have curvature order $\leqq a$ at $p$. Then given $A>0$ there exists $r>0$ such that

$$
\int_{1}^{r} k_{v}(t) t^{a-1} d t \geqq A \quad \text { for all } \quad v \in S(p) .
$$

Proof. Let $k$ be the real-valued function on $S H$ such that $k(v)=$ $\min \{|K(\pi)|: v \in \pi\}$. A standard argument shows that $k$ is continuous. Since the function $S H \times R \rightarrow S H:(v, t) \rightarrow \gamma_{v}^{\prime}(t)$ is smooth it follows that the function $S H \times R \rightarrow R:(v, t) \rightarrow k\left(\gamma_{v}^{\prime}(t)\right)=k_{v}(t)$ is continuous. For fixed $r$ its restriction to $S(p) \times[0, r]$ is uniformly continuous, and hence the function $I_{r}: S(p) \rightarrow R$ such that $I_{r}(v)=\int_{1}^{r} k_{v}(t) t^{a-1} d t$ is continuous. The result follows.

The following is a refinement of Lemma 9.10 of [3].

Proposition 5.9. Let $H$ be a Hadamard manifold. If $H$ has curvature order $\leqq 2$ at a point $p$, that is, if

$$
\int_{1}^{\infty} k_{v}(t) t d t=\infty \quad \text { for all } \quad v \in S(p)
$$

then $H$ satisfies Axiom 1.

Proof. Given $\varepsilon>0$ choose $r$ by the preceding lemma so that $\int_{1}^{r} k_{v}(t) t d t \geqq \pi / \varepsilon$ for all $v \in S(p)$. Let $\sigma$ be a geodesic segment from $m$ to $n$ in $H$ such that $d(p, \sigma) \geqq r$. We show that $\Varangle_{p}(m, n) \leqq \varepsilon$. Let $\Sigma$ be the radial surface from $p$ to $\sigma$ with parametrization $\boldsymbol{x}$ as defined earlier. By Proposition 5.5, $\pi \geqq \iint_{\Sigma}\left|G^{*}\right| d A$, where $G^{*}$ is the Gaussian curvature of $\Sigma$. By a lemma of Synge, $G^{*} \leqq K \leqq 0$, where $K$ is evaluated on the tangent plane of $\Sigma$. Thus $\pi \geqq \iint_{\Sigma}|K| d A$. Let us write $k_{u}(t)$ for $k_{w}(t)$ where $w=\rho_{u}^{\prime}(0)$. Then $|K|$ at $\boldsymbol{x}(t, u)$ is $\geqq k_{u}(t)$. For $\boldsymbol{x}$ we have $E=1, F=0$ and by Lemma $5.6(4), \sqrt{G(t, u)} \geqq t\left\|\pi \sigma^{\prime}(u)\right\|$. Hence

$$
\begin{aligned}
\pi & \geqq \iint_{\Sigma}|K| d A \geqq \int_{0}^{b}\left\{\int_{1}^{r} k_{u}(t) t d t\right\}\left\|\pi \sigma^{\prime}(u)\right\| d u \\
& \geqq \frac{\pi}{\varepsilon} \Theta(p, \sigma) \geqq \frac{\pi}{\varepsilon} \succ_{p}(m, n) .
\end{aligned}
$$

We now give examples showing that Axioms 1 and 2 are independent. The first of these will be useful in later developments. 
ExAmPle 5.10. A Hadamard manifold satisfying Axiom 1 but not Axiom 2.

Let $H$ be the plane $R^{2}$ with the Riemannian structure $E=1$, $F=0, G=g^{2}(u)$ where $g^{\prime \prime} \geqq 0$ and

$$
g(u)=\left\{\begin{array}{l}
1 \text { for }|u| \leqq 1 \\
\cosh u \text { for }|u| \geqq 2 .
\end{array}\right.
$$

Then $H$ is a Hadamard manifold for which the vertical strip $|u| \leqq$ 1 is Euclidean, but $K \equiv-1$ for $|u| \geqq 2$. A Clairaut argument as in Example 5.3 shows that every geodesic starting at the origin, except parametrizations of the $v$-axis, eventually leaves the strip $|u| \leqq 2$. Thus $\int_{1}^{\infty} k_{w}(t) t d t=\infty$ for all but two unit vectors at the origin.

By modifying the proof of Proposition 5.9 it can be shown that, in spite of these two exceptions, Axiom 1 holds for $H$. However Axiom 2 fails since $H$ contains a flat strip.

If we make the further assumption that $g(u)=g(-u)$ then the maps $(u, v) \rightarrow(-u, v+1)$ and $(u, v) \rightarrow(u, v+1)$ are isometries of $H$. Hence we can induce metrics on the Möbius band and cylinder that satisfy Axiom 1 but not Axiom 2.

To see that there are Hadamard manifolds satisfying Axiom 2 but not Axiom 1, we note that one can easily construct two-dimensional manifolds with $K<0$ and finite total curvature. However, if $H$ satisfies Axiom 1 it has infinite total curvature. In fact, for each $n \geqq 3$ divide $H$ into equiangular sectors $\Delta_{i}, 1 \leqq i \leqq n$, by means of geodesics from a fixed point $p \in H$ to points $x_{i} \in H(\infty)$. By Axiom 1 there is a geodesic joining $x_{i}$ to $x_{i+1}$. By the Gauss-Bonnet theorem $\iint_{A_{i}}|K| d A \geqq \pi-(2 \pi / n)$, so that

$$
\iint_{H}|K| d A \geqq(n-2) \pi
$$

If there are severe restrictions on $H$, for example if $H$ covers a compact manifold in the Riemannian sense, then it is unknown if Axioms 1 and 2 are independent in $H$.

Proposition 5.11. If $H$ is a Hadamard manifold such that $\int_{0}^{\infty} k_{v}(t) d t=\infty$ for every $v \in S H$ then $H$ satisfies the Zero Axiom.

The proof is a tedious computation which we omit since we do not use the result.

6. Axial isometries. In this section and the next we study the isometries of a Hadamard manifold $H$ both individually and as elements of properly discontinuous groups, with emphasis on the fixed 
points at infinity. If $\varphi$ is an isometry of $H$ the displacement function $g_{\varphi}(p)_{1}^{\prime}=d(p, \varphi p)$ divides the isometry group of $H$ into three classes. $\phi$ is said to be elliptic if $g_{\varphi}$ has minimum zero, axial if $g_{\varphi}$ has positive minimum and parabolic if $g_{\varphi}$ has no minimum. Hence elliptic isometries are those with fixed points in $H$. Since our main interest lies in properly discontinuous groups we shall consider only nonelliptic isometries. Hence $g_{\varphi}$ will always be a smooth convex function [3].

It is well known that $\varphi$ is axial if and only if $\varphi$ translates a geodesic $\gamma$, that is $\varphi(\gamma t)=\gamma(t+a)$ for all $t \in \boldsymbol{R}$ and some $a \neq 0$. (The geodesic $\gamma$ is then called an axis of $\varphi_{\text {.) }}$ Furthermore $p \in H$ is a minimum point of $g_{\varphi}$ if and only if $\varphi$ translates the geodesic $\gamma_{p, \varphi p}$. Hence the minimum set of $g_{\varphi}$ is the union of the images of all geodesics translated by $\phi$ and each is translated by $\pm \min g_{\varphi}$.

Using the same notation for an isometry of $H$ and its asymptotic extension to $\bar{H}$ we obtain the following result.

Lemma 6.1. Every (non-elliptic) isometry $\varphi$ of $H$ has a fixed point in $H(\infty)$.

Proof. Since $\bar{H}$ is a topological $n$-cell we may apply the Brouwer fixed point theorem.

The result below gives a useful way to find fixed points in $H(\infty)$.

Lemma 6.2. Let $\varphi$ be an isometry of $H . \quad$ If $p \in H$ then any accumulation point in $H(\infty)$ of the set $\left\{\varphi^{n}(p): n \in Z\right\}$ is a fixed point of $\varphi$.

Proof. Suppose that $\left\{\varphi^{n_{k}}(p)\right\} \rightarrow x \in H(\infty)$ as $k \rightarrow \infty$. By continuity $\left\{\varphi^{n_{k}+1}(p)\right\} \rightarrow \varphi(x)$ as $k \rightarrow \infty$. But

$$
d\left(\varphi^{n_{k}+1}(p), \varphi^{n_{k}}(p)\right)=d(\varphi p, p) .
$$

Hence by the law of cosines $\Varangle_{p}\left(\varphi^{n} k(p), \varphi^{n_{k}+1}(p)\right) \rightarrow 0$ as $k \rightarrow \infty$, and $\varphi(x)=x$.

We note that by the law of cosines the set of infinite accumulation points in the preceding lemma is independent of the choice of the point $p$. In general we will not obtain all fixed points of $\varphi$ in this manner.

ExAmPle 6.3. Let $H$ be the Euclidean space $R^{n}$, and let $\varphi$ be a translation. Then $\left\{\varphi^{n}(0): n \in Z\right\}$ has only two accumulation points in $R^{n}(\infty)$, the endpoints of the axis of $\varphi$ which passes through the origin. However, $\varphi$ fixes every point of $R^{n}(\infty)$ since it maps every geodesic to a parallel geodesic.

In an arbitrary Hadamard manifold the preceding example shows that the fixed point set of a nonelliptic isometry $\phi$ gives only a very 
weak description of the geometrical character of $\varphi$. If Axiom 1 is imposed on $H$ the situation changes dramatically and we obtain the following fundamental result.

Proposition 6.4. Let $H$ satisfy Axiom 1. If a non-elliptic isometry fixes distinct points $x$ and $y$ of $H(\infty)$, then $\varphi$ translates a geodesic joining $x$ to $y$. Furthermore $x$ and $y$ are the only fived points of $\varphi$.

We shall see in a moment that $\varphi$ may translate many geodesics joining $x$ and $y$. The following argument uses some facts about convex functions drawn from the beginning of $\S 11$.

Proof. That $\varphi$ fixes $x \in H(\infty)$ means that $\varphi_{\circ} \alpha$ is asymptotic to $\alpha$ for all $\alpha \in x$. Hence for each $\alpha \in x$ we have $g_{\varphi}(\alpha t)=d(\alpha t, \varphi \alpha t) \leqq C$ for all $t \geqq 0$. Thus the convex function $g_{\varphi}$ is monotone decreasing on $x$ and similarly on $y$. It then follows from Proposition 11.3 that $g_{0}$ has a minimum, and in fact that there exists a geodesic $\gamma$ joining $x$ and $y$ with $\gamma \subseteq \operatorname{Min}(f)$.

Next we show that $\varphi$ translates $\gamma$. Since $g_{\varphi}$ has a minimum at $\gamma(0), \varphi$ translates the geodesic $\beta$ from $\gamma(0)$ through $\varphi \gamma(0)$. By Corollary 4.5 we may choose $C>0$ such that $d(\alpha, \beta) \leqq C$ for any geodesics $\alpha, \beta$ joining $x$ to $y$. Then $d(\beta(n a), \gamma)=d\left(\Phi^{n} \gamma(0), \gamma\right)=d\left(\Phi^{n} \gamma, \gamma\right) \leqq C$ for any positive integer $n$. Hence $\beta(\infty)=\gamma(\infty)$. It follows that $\beta=$ $\gamma$ since both geodesics contain $\gamma(0)$.

Finally $x$ and $y$ are the only fixed points of $\varphi$. If $z$ were a third, then $\varphi$ would translate a geodesic from $x$ to $z$ by the arguments above. However we shall prove in Proposition 6.7 (3) that any two axes of $\varphi$ have the same endpoints.

We can restate this result in the following form, reminiscent of a definition from automorphic function theory.

Theorem 6.5. Let $H$ satisfy Axiom 1. Then every non-elliptic isometry of $H$ has at most two fixed points in $H(\infty)$ : one if parabolic and two if axial.

In automorphic function theory one classifies the linear fractional transformations that preserve the disc by the number of their fixed points on the unit circle. Theorem 6.5 is the key to further generalizations of many classical results of automorphic function theory and of geodesic flows [7].

If Axiom 1 holds then it follows by Proposition 6.7 and the results above that for a nonelliptic isometry $\varphi$ of $H$ the sequences $\left\{\varphi^{n}(p)\right\}$, $n \geqq 0$ and $\left\{\varphi^{-n}(p)\right\}, n \geqq 0$ converge to the fixed points of $\varphi$ if $\varphi$ generates a properly discontinuous group. 
Let $\varphi$ be an isometry of $H$ with fixed point $x \in H(\infty)$. Then $\varphi$ carries any limit sphere at $x$ into another limit sphere at $x$. To each such $\varphi$ we associate the signed displacement $T_{x}(\varphi)$ which is positive if $\varphi$ expands limit spheres at $x$, negative if it contracts them and zero if it preserves them. This gives another tool for the study of the fixed points of $\varphi$.

More explicitly, let $x \in H(\infty)$ be fixed by an isometry $\varphi$ of $H$ and let $f_{\gamma}$ be a Busemann function at $x$. Then $f_{\gamma} \circ \varphi=f_{\varphi-1 \gamma}$. In fact $f_{\varphi-1_{\gamma}}(p)=\lim _{t \rightarrow \infty} d\left(p, \varphi^{-1} \gamma t\right)-t=\lim _{t \rightarrow \infty} d(\varphi p, \gamma t)-t=f_{\gamma}(\varphi p)$. By Proposition 3.1 there exists a well-defined number $T_{x}(\varphi)$ which is the value of the constant function $f \circ \phi-f$ for every Busemann function $f$ at $x$. By Corollary 3.3, $\left|T_{x}(\varphi)\right|=|f \varphi p-f p|=d(L(\varphi p, x), L(p, x))$, and hence $T(\varphi)$ is the signed distance from the limit sphere $L(p, x)$ to the limit sphere $L(\Phi p, x)$. The following are clearly equivalent: (1) $T_{x}(\varphi)=0$, (2) $f \circ \varphi=f$ for every Busemann function $f$ at $x$, (3) $\varphi(L)=L$ for every limit sphere $L$ at $x$, (4) $\varphi \circ \eta_{L}=\eta_{L} \circ \varphi$, where $\eta_{L}$ is the projection on $L$.

Proposition 6.6. (1) If $x \in H(\infty)$ is a fixed point of $\varphi$ then $\left|T_{x}(\varphi)\right| \leqq \inf g_{\varphi}$. Furthermore if $x$ is an endpoint of an axis $\alpha$ of $\varphi$ then $\left|T_{x}(\varphi)\right|=\min g_{\varphi}$.

(2) Let $I_{x}(H)$ be the group of isometries of $H$ that fix $x \in H(\infty)$. Then $T_{x}: I_{x}(H) \rightarrow R$ is a homomorphism into the additive group of real numbers.

Proof. (1) By Proposition 3.2, for any point $p \in H$ we have $g_{\varphi}(p)=d(p, \varphi p) \geqq d(p, L(\varphi p, x))=|f(p)-f(\varphi p)|=\left|T_{x}(\varphi)\right|$. In the axial case, if $\varphi(\alpha 0)=\alpha(\alpha)$ then $\varphi(\alpha 0)$ is the projection of $\alpha(0)$ on $L(\varphi \alpha 0, x)=L(\alpha a, x)$. Hence $|\alpha|=\min g_{\varphi}=d(\alpha 0, L)=\left|T_{x}(\varphi)\right|$.

(2) $T_{x}(\varphi \circ \psi)=f \circ \varphi \circ \psi-f=[(f \circ \varphi-f) \circ \psi]+[f \circ \psi-f]=T_{x}(\varphi)+$ $T_{x}(\psi)$.

In the remainder of this section we consider axial isometries, leaving parabolic isometries for the next section. If $\phi$ is an element of a properly discontinuous group $D$, then an axis $\alpha$ of $\varphi$ in $H$ projects to a closed geodesic $\pi \circ \alpha$ in $H / D$. Conversely, if $\gamma$ is a closed geodesic in $H / D$ then every lift of $\gamma$ to $H$ is an axis of some element $\varphi \in D$. Example 6.3 shows that an isometry $\varphi$ may have many different axes. We now collect some elementary axial properties.

Proposition 6.7. If $\varphi$ is an axial isometry of $H$ then

(1) $\varnothing$ generates a properly discontinuous group.

(2) If $\alpha$ is an axis of $\phi$ then for a suitable orientation of $\alpha$, $\varphi^{-n}(p) \rightarrow \alpha(-\infty)$ and $\varphi^{n}(p) \rightarrow \alpha(\infty)$ for any point $p \in H$ as $n \rightarrow \infty$. 
(3) All axes of $\varphi$ are equivalent, that is, they have the same endpoints.

(4) If $\varphi$ translates $\alpha$ and $\beta$, then $\varphi$ translates every longitudinal geodesic in the flat strip $[\alpha, \beta]$.

(5) If an isometry $\psi$ commutes with a nontrivial power of $\varphi$, then $\psi$ fixes the two endpoints of the axes of $\varphi$.

Proof. (1) Let $\varphi$ translate a geodesic $\alpha$ by $a \neq 0$. If $p \in H$ let $\alpha(t)$ be the foot of $p$ on $\alpha$. Then $\varphi^{n}(p)$ has foot $\varphi^{n}(\alpha t)=\alpha(t+n a)$ on $\alpha$. Since perpendiculars from a geodesic spread apart in $H$, $d\left(p, \varphi^{n} p\right) \geqq d(\alpha(t), \alpha(t+n a))=|n a| \geqq|a|$ for $n \neq 0$.

(2) Let $\varphi$ translate $\alpha$ by $a \neq 0$. Reversing the orientation of $\alpha$ if necessary, we may assume that $a>0$. For every integer $n$ we have $d\left(\Phi^{n} p, \alpha(n a)\right)=d(p, \alpha(0))$, and the result follows by the law of cosines.

(3) follows immediately from (2).

(4) Parametrize $\alpha$ and $\beta$ so that $d(\alpha t, \beta t)=d(\alpha, \beta)$ for all $t \epsilon$ $R$. If $p \in[\alpha, \beta]$ lies on the perpendicular transversal from $\alpha(t)$ to $\beta(t)$, then $\varphi(p)$ lies on the transversal from $\alpha(t+a)$ to $\beta(t+a)$. Therefore $[\alpha, \beta]$ is invariant under $\varphi$. Since $d(p, \alpha)=d(\varphi p, \alpha)$ it follows that $\varphi(p)$ lies on the longitudinal geodesic through $p$, and hence $\varphi$ translates the (Sp) longitudinal geodesic $\gamma_{p, \varphi p}$ with displacement $a$.

(5) Suppose $\psi \varphi^{n}=\phi^{n} \psi$. We may assume that $n>0$. If $\varphi$ translates $\alpha$ by $a>0$ then $\varphi^{n}$ translates $\alpha$ by $n a>0$. Since $\phi^{n} \psi(\alpha t)=$ $\psi \varphi^{n}(\alpha t)=\psi(\alpha(t+n a))$ it follows that $\phi^{n}$ translates $\psi \circ \alpha$ by $n a$. By the argument of (2) the result follows.

Proposition 6.8. Let $\alpha$ be an axis of an isometry $\varphi$ of $H$ with endpoints $x$ and $y$. Let $\psi$ be an isometry of $H$ that fixes one of these endpoints and such that is and $\varphi$ generate a properly discontinuous group. Then 's commutes with a power of $\varphi$, and hence fixes the other endpoint of $\alpha$.

Proof. Let $\psi(y)=y$ for example. Orient $\alpha$ so that $\alpha(\infty)=y$, and if necessary replace $\varphi$ by $\varphi^{-1}$ so that $\varphi$ translates $\alpha$ by $a>0$. Let $p=\alpha(0)$ and for an integer $n>0$ consider $d\left(p, \varphi^{-n} \psi \varphi^{n} p\right)=$ $d\left(\phi^{n} p, \psi \varphi^{n} p\right)=d(\alpha(n \alpha), \psi \alpha(n \alpha))$. Since $\psi(y)=y, \alpha$ and $\psi \circ \alpha$ are asymptotes and there exists $C>0$ such that $d\left(p, \varphi^{-n} \psi \varphi^{n} p\right) \leqq C$ for every $n>0$. By proper discontinuity, $\varphi^{-n} \psi \varphi^{n}=\varphi^{-m} \psi \varphi^{m}$ for integers $m \neq$ $n$. Thus $\psi$ commutes with $\varphi^{m-n}$. By the preceding proposition $\psi$ fixes $x$ also.

If $\psi$ is known to be axial we can strengthen Proposition 6.8 as follows.

Proposition 6.9. Let $\phi$ and $\psi$ be axial isometries of $H$ that generate a properly discontinuous group. If $\varphi$ and $\psi$ have asymptotic 
axes, then there exist integers $m, n$ such that $\varphi^{m}=\psi^{n}$. Hence the axes of $\varphi$ and $\psi$ are equivalent.

Proof. Orient the axes $\alpha$ and $\beta$ so that $\alpha(\infty)=\beta(\infty)$. Replacing $\phi$ and $\psi$ by their inverses if necessary we assume that $\phi$ translates $\alpha$ by $a>0$ and $\psi$ translates $\beta$ by $b>0$. For each integer $n \geqq 1$ let $\beta\left(t_{n}\right)$ be the foot of $\alpha(n a)$ on $\beta$. Then there exists an integer $m=$ $m(n)$ such that $\left|t_{n}-m b\right|<b$. Now $d\left(\alpha 0, \psi^{-m} \varphi^{n} \alpha 0\right)=d\left(\psi^{m} \alpha 0, \varphi^{n} \alpha 0\right) \leqq$ $d\left(\psi^{m} \alpha 0, \psi^{m} \beta 0\right)+d\left(\beta(m b), \beta\left(t_{n}\right)\right)+d\left(\beta\left(t_{n}\right), \alpha(n a)\right)$. If $C=d(\alpha 0, \beta 0)$ then $d(\alpha t, \beta) \leqq C$ for all $t \geqq 0$, and hence $d\left(\alpha 0, \psi^{-m} \varphi^{n} \alpha 0\right) \leqq b+2 C$. The result follows by proper discontinuity.

We now consider the simplest of the three types of manifolds mentioned in the Introduction.

DeFinition 6.10. Let $D$ be a properly discontinuous group of isometries of $H$. We say that $D$ (and the manifold $H / D$ ) are axial if there are distinct points $x, y \in H(\infty)$ such that every $1 \neq \varphi \in D$ translates a geodesic from $x$ to $y$.

Hence $D$ is axial if every $1 \neq \varphi \in D$ is axial and all axes are equivalent. In view of Proposition 6.9 it would suffice to require that all axes be asymptotic.

If Axiom 2 holds for $H$, then every element of an axial group $G$ translates the same geodesic and it is well known that $G$ is infinite cyclic. Our aim now is to prove:

THEOREM 6.11. An axial group $D$ on an arbitrary Hadamard manifold $H$ is infinite cyclic.

The following argument is a modification of the proof of a more general result in [9].

Lemma 6.12. Let $\varphi$, $\psi$ be isometries of $H$ that translate a geodesic $\gamma$. Let $C_{\varphi}, C_{\psi}$ be the minimum sets of the displacement functions $g_{\varphi}, g_{\psi}$ respectively. Then either $C_{\varphi} \supseteqq C_{\psi}$ or $C_{\varphi} \cap C_{\psi}$ is nowhere dense in $C_{\psi}$.

Proof. Let $\beta$ be a geodesic equivalent to $\gamma$. We show that either $\phi$ translates $\beta$ or the intersection of the flat strips $[\beta, \gamma]$ and $[\rho \beta, \gamma]$ is $\gamma$. Suppose $p \notin \gamma$ lies in the intersection of both strips, and let $q$ be the foot of $p$ on $\gamma$. Let $c=d(\beta, \gamma)=d(\varphi \beta, \gamma)$. Let $\sigma:[0, c] \rightarrow$ $H$ be the unit speed geodesic segment such that $\sigma(0)=q$ and $\sigma$ passes through $p$. Then $\sigma$ is a perpendicular transversal in both strips, and $\sigma(c)$ lies in $\varphi \beta$ and $\beta$. Hence $\varphi$ translates $\beta$.

Suppose that $C_{\varphi} \cap C_{\psi}$ contains an open set $U$ of $C_{\psi}$. Let $p \in U$ and let $\alpha$ be the geodesic through $p$ translated by $\psi$. Since the axes 
of $\varphi$ and $\psi$ are equivalent, $\varphi$ translates $\alpha$. Let $\beta$ be any axis of $\psi$. In the flat strip $[\beta, \alpha]$ an initial segment of the perpendicular transversal starting at $p$ lies in $C_{\varphi}$. Hence $\phi$ translates longitudinal geodesics in $[\beta, \alpha]$ that are close to $\alpha$. Since the intersection of $[\beta, \alpha]$ and $[\varphi \beta, \alpha]$ is larger than $\alpha$, the preceding remark implies that $\varphi$ translates $\beta$. Thus $C_{\varphi} \supseteqq C_{\psi}$.

An element $\psi$ of a group $D$ is a root of $\phi \in D$ if $\psi^{k}=\phi$ for some integer $k$. If $|k| \geqq 2$ then $\psi$ is a proper root; $\varphi$ is rootless if it has no proper roots in $D$.

Lemma 6.13. Let $D$ be an axial group. Then every element of $D$ has a rootless root.

Proof. Let $1 \neq \varphi \in D$. The closed set $C_{\varphi}$ is convex, and by [6] any closed (totally) convex subset of $H$ is a topological manifold of dimension $k \leqq \operatorname{dim} H$. Let $\gamma$ be an axis of $\varphi$, and let $\psi$ generate the cyclic subgroup of $D$ that translates $\gamma$. Since $\psi$ is a root of $\phi$ it suffices to show that $\psi$ has a rootless root. If $\eta$ is a proper root of $\psi$, then $\eta$ does not translate $\gamma$ and by Lemma $6.12 C_{\eta}$ is nowhere dense in $C_{\psi}\left(C_{\eta} \subseteq C_{\psi}\right.$, since any axis of $\eta$ is an axis of $\left.\psi\right)$. Thus $\operatorname{dim} C_{\eta}<\operatorname{dim} C_{\psi}$. Since $\operatorname{dim} C_{\psi}$ is finite the process of extracting proper roots must terminate in a rootless root of $\varphi$.

To prove Theorem 6.11 we first note that $D$ is abelian; in fact if $x$ and $y$ are the endpoints of the axes of $D$ then, by Proposition 6.6, $T_{x}$ is a homomorphism of $D$ into $R$ whose kernel is the identity. By the preceding lemma, $D$ contains a rootless element $\varphi \neq 1$. We assert that $\varphi$ generates $D$. Let $\psi \in D$. By Proposition 6.9 we conclude $\left({ }^{*}\right), \varphi^{m}=\psi^{n}$ for some $m, n$. It is well known that $D \approx \pi_{1}(H / D)$ is torsionfree, and we can thus assume that $m$ and $n$ are relatively prime. Then $m p+n q=1$ for some $p, q$. Hence by $\left(^{*}\right)$ we obtain $\varphi=\psi^{n p} \varphi^{n q}=\left(\psi^{p} \varphi^{q}\right)^{n}$. Since $\varphi$ is rootless, $n= \pm 1$. Hence by $(*)$ we conclude that $\psi$ is a power of $\varphi$.

We give a sufficient condition for $D$ to be axial.

Proposition 6.14. Let $H$ be geodesically bounded (\$4) and let $D$ be a properly discontinuous group of isometries of $H$ with a central axial element. Then $D$ is axial.

Proof. Let $\varphi$ be a central axial element, and let $\alpha$ be an axis of $\varphi$ with endpoints $x$ and $y$. If $\varphi$ fixed a third point $z \in H(\infty)$, then $g_{\varphi}$ would have a minimum on every point of $\gamma_{p z}$ where $p$ is a point of $\alpha$. Hence the axes of $\varphi$ would not meet a compact set in $H$, contradicting the assumption that $H$ is geodesically bounded. Thus $x$ and $y$ are the only fixed points of $\varphi$. 
Since $\phi$ is central the displacement function $g_{\varphi}$ induces a convex function $f$ on $H / D$ such that $g_{\varphi}=f \circ \pi$, where $\pi: H \rightarrow H / D$. The minimum set of $f$ is always totally convex and is compact since $H$ is geodesically bounded. By Proposition 10.15 of [3] every element of $D$ is axial. Let $\xi \in D$ and let $\beta$ be an axis of $\xi$. Since $\varphi$ is central, $\varphi$ fixes the endpoints of $\beta$ by Proposition 6.7 (5). Hence $\beta$ joins $x$ to $y$ by the remark above, and $D$ is axial.

REMARK. The result fails if the central element is parabolic or if $H$ is not geodesically bounded. If $T$ is a flat torus $R^{2} / D$, then $D \approx \pi_{1}(T)$ is an abelian group of axial isometries. However $R^{2}$ is not geodesically bounded, and $T$ is not axial. From [3] we see that $M=$ $R \times T$ is a parabolic manifold with $K \equiv-1 . \quad H^{3}$ is geodesically bounded, but $M$ is not axial.

Proposition 6.15. If $M=H / D$ is axial, then every closed geodesic $\gamma$ of $M$ is simply closed. Furthermore, if $\gamma$ has minimum period its image $\gamma(R)$ is totally convex.

Proof. If $\gamma$ is a closed geodesic of period $c$ in $M$ then $\gamma$ has a lift $\alpha$ that is translated a distance $c>0$ by some $\phi \in D$. If $\gamma$ is not simply closed there exists $\psi \in D$ and numbers $0 \leqq a<b \leqq c$ such that $\psi(\alpha a)=\alpha b$ and $b-a<c$. By hypothesis, is fixes $\alpha(\infty)$. Since $\alpha$ and $\psi \circ \alpha$ are asymptotes with the point $\alpha b$ in common, $\psi \circ \alpha$ is a re parametrization of $\alpha$; hence $\psi(\alpha t)=\alpha(t+b-a)$ for all $t$. Since $b-a<c$ this contradicts the fact that $\gamma$ has period $c$.

That $\gamma$ has minimum period means $\gamma$ has a lift $\alpha$ that is translated by a generator $\varphi$ of $D$. Let $\sigma$ be a geodesic segment in $M$ with endpoints in $\gamma R$, say $\sigma(0)=\gamma(0)$ and $\sigma(a)=\gamma(b)$. Let $\tau$ be the lift of $\sigma$ such that $\tau(0)=\alpha(0)$. Since $D$ is cyclic, there is an integer $n$ such that $\varphi^{n} \alpha b=\tau a$. It follows that $\tau$ lies in $\alpha R$; hence $\sigma$ lies in $\gamma R$.

Corollary 6.16. If $M=H / D$ is an axial manifold, then $M$ is a smooth vector bundle over a circle.

Proof. The preceding result shows that if $\gamma$ is a closed geodesic of $M$ having minimum period, then $C=\gamma(R)$ is a totally convex imbedded circle. It follows from [3] that the exponential map is a diffeomorphism of the normal bundle of $C$ onto $M$.

If $H$ satisfies Axiom 2, then an axial manifold contains a unique closed geodesic, the projection of the unique axis of $D$. If Axiom 2 fails there will in general be infinitely many closed geodesics, even if Axiom 1 holds. However these geodesics are strongly related as we now see. Recall that geodesics in $H$ are equivalent if they have 
the same endpoints, regardless of orientation. As with the asymptote relation we say that geodesics $\alpha$ and $\beta$ of $M=H / D$ are equivalent if they have lifts to $H$ that are equivalent. This is indeed an equivalence relation on the geodesics of $M=H / D$ which is trivial when Axiom 2 holds. It follows by Proposition 6.9 that closed geodesics $\alpha$ and $\beta$ in $M$ are equivalent if and only if they are asymptotic.

REMARK 6.17. If $A$ is an equivalence class of closed geodesics in $M=H / D$ (not necessarily axial) then any two elements of $A$ are homotopic by means of a flat, totally geodesic homotopy whose intermediate curves are also elements of $A$.

Proof. (Compare 3.5 of [3].) Elements $\alpha, \beta$ of $A$ have equivalent lifts $\alpha^{*}, \beta^{*}$ to $H$. Let $\varphi$ translate $\alpha^{*}$ and $\psi$ translate $\beta^{*}$. By Proposition $6.9 \varphi^{m}=\psi^{n}=\sigma$ for suitable integers $m, n$. Since $\sigma$ translates $\alpha^{*}$ and $\beta^{*}$, by Proposition 6.7 (4) $\sigma$ translates each longitudinal geodesic of the flat, totally geodesic strip $\boldsymbol{x}$ with boundary $\alpha^{*}, \beta^{*}$. Thus $\pi \circ \boldsymbol{x}$ is the desired homotopy.

The elements of an equivalence class of closed geodesics in $M=$ $H / D$ may have different periods even if $H$ satisfies Axiom 1.

EXAMPLE 6.18. Let $H$ be the Hadamard manifold in Example 5.10 , so that $H$ satifies Axiom 1. Let $\varphi$ be the isometry $\varphi(u, v)=$ $(-u, v+1)$. Then $\varphi$ translates only the $v$-axis $\beta$, but $\varphi^{2}$ translates each element in the set $A^{*}$ of vertical geodesics in the strip $|u| \leqq 1$. If $D$ is the group generated by $\varphi$ then $M=H / D$ is axial, an open Möbius band. The equivalence class $A$ of closed geodesics of $M$ is the projection of $A^{*}$ and the union of all geodesics in $A$ is a flat Möbius band with boundary. The central closed geodesic $\pi \circ \beta$ has period 1, while all others have period 2.

Even if $M$ is orientable the closed geodesics in an equivalence class may have different periods. For an integer $k$ let $\eta$ be the rotation of $R^{2}$ through the angle $2 \pi / k$. On $R^{3}=R^{2} \times R$ let $\varphi(p, t)=$ $(\eta p, t+1)$ and let $D$ be the group generated by $\varphi$. The geodesics normal to the $x y$ plane project to an equivalence class of closed geodesics in $M=H / D$. The projection of the $z$-axis has period 1 , while all others have period $k$.

7. Parabolic isometries. At most points of comparison the behavior of a parabolic isometry $\varphi$ of a Hadamard manifold $H$ can be considerably more complicated than that of an axial isometry; we have no direct link between $\varphi$ and the geometry of $H$ comparable with the notion of axis. For example, in the preceding section we saw that a group $G$ of axial isometries with common fixed point in 
$H(\infty)$ must be infinite cyclic. By contrast if $G$ consists of parabolic isometries Proposition 9.1 shows that a common fixed point imposes no algebraic restriction whatsoever on $G$.

We begin this section by considering the geometrical significance in $H / D$ of the horocyclic limit set of $D$. The importance of limit spheres in the study of parabolic isometries is illustrated by Proposition 7.8 which asserts that a parabolic isometry $\varphi$ preserves the limit spheres at some fixed point $x \in H(\infty)$. This strengthens the analogy between $\varphi$ and a rotation of $H$ about a finite point, and leads to a satisfactory structure theorem (7.10) for parabolic manifolds, the second of the three types mentioned in the Introduction. (Theorem 7.10 shows that the common fixed point condition has strong geometric-if not algebraic-consequences.) Finally under mild conditions we show that if $x \in H(\infty)$ is a parabolic fixed point, then the geodesics of $\pi_{a}(x)$ are divergent in $M$. The scheme of the proof is simple: an isometry $\varphi$ of $H$ determines a free class of loops in $M: F(\varphi)=\left\{\pi \circ \gamma_{p, \varphi p} \mid p \in H\right\}$. If $\varphi$ is parabolic then $F(\varphi)$ has no shortest element (closed geodesic); hence a minimizing sequence in $F(\varphi)$ must escape to infinity-along the projection of the fixed point $x$.

Recall from $\S 1$ that the projection $\pi: H \rightarrow M=H / D$ induces a projection $\pi_{a}$ of $H(\infty)$ onto the set $A(M)$ of asymptote classes of $M$. Thus for $x \in H(\infty)$ we can study $\pi_{a}(x)$ in terms of the relation between $x$ and $D$. The following generalizations of the notion of minimizing geodesic are basic:

Definition 7.1. A (unit speed) geodesic $\gamma$ in $M$ is (1) almost minimizing if there is a number $c>0$ such that $d(\gamma 0, \gamma t) \geqq t-c$ for all $t$, and (2) ultimately minimizing if there exists a number $a$ such that $\gamma \mid[a, \infty)$ is minimizing, that is, $d(\alpha a, \alpha t)=t-a$ for all $t \geqq \alpha$.

We say that an asymptote class $y \in A(M)$ is [almost, ultimately] minimizing if every geodesic $\gamma \in y$ is [almost, ultimately] minimizing. Further, $x \in H(\infty)$ is [almost, ultimately] D-minimizing if $\pi_{a}(x)$ is [almost, ultimately] minimizing.

Obviously, ultimately minimizing implies almost minimizing, but the example below shows that the converse is false (at least for noncompact surfaces). Any geodesic asymptotic to an almost minimizing geodesic is itself almost minimizing. We do not know whether this asymptote property holds for ultimately minimizing geodesics. Nevertheless asymptote classes of both kinds arise naturally as we shall see.

EXAMPLE 7.2. A geodesic (in a Visibility manifold) that is almost minimizing but not ultimately minimizing.

Let $\alpha, \beta, \gamma$ be mutually orthogonal geodesics starting at the origin of the Poincaré ball model $P^{3}$ of hyperbolic 3 -space. Let $P_{\alpha \beta}$ 
be the totally geodesic surface containing $\alpha$ and $\beta$ (so $P_{\alpha \beta}$ is a hyperbolic plane). For each $\varepsilon>0$ let $C_{\varepsilon}$ be a $C^{\infty}$ convex curve in $P_{\alpha \beta}$ that (a) starts at $\alpha(\varepsilon)$, (b) contains the segment $\alpha([\varepsilon / 2, \varepsilon])$ and (c) asymptotically approaches $\beta$. Revolving $C_{\varepsilon}$ about $\beta$ produces a surface $T_{\varepsilon}$ with boundary $\partial T_{\varepsilon}$ a hyperbolic circle of radius $\varepsilon$, base an annulus of radii $\varepsilon / 2, \varepsilon$ in $P_{\alpha \gamma}$, and Gaussian curvature $K \leqq-1$ (by the Gauss equation for $T_{\varepsilon} \subset P^{3}$ ). Call $T_{\varepsilon}$ a contracting tube. (If instead of (c), $C$ eventually curves away from $\beta, T_{\varepsilon}$ is an expanding tube.) We can attach $T_{\varepsilon}$ to a hyperbolic plane $P$ at a point $p$ by deleting the $\varepsilon$-neighborhood $N$ of $p$ and fitting $T_{\varepsilon}$ in an obvious way around the boundary of $N$. The result is a complete $K \leqq-1$ surface.

To construct the surface used for the example, fix a geodesic $\alpha$ in the plane $P$, and for each $n \geqq 1$ let $T_{n}$ be a tube whose boundary $\partial T_{n}$ has radius $1 / 2^{n}$. Let $M$ be the (complete, $K \leqq-1$ ) surface obtained by attaching each $T_{n}$ to $P$ so that $\partial T_{n}$ is tangent to $\alpha$ at $\alpha(n)$ and is above [below] $\alpha$ for $n$ odd [even]. By a limit argument there is a geodesic $\beta$ in $M$ such that $\beta(0)=\alpha(0)$ and $\beta$ passes above [below] the tube $T_{n}$ for $n$ odd [even]. (Necessarily $\beta$ will rise up each tube somewhat.) For each $n, \beta$ clearly crosses $\alpha$ at a unique point $\beta\left(t_{n}\right)=\alpha\left(s_{n}\right)$ such that $n<s_{n}<n+1$.

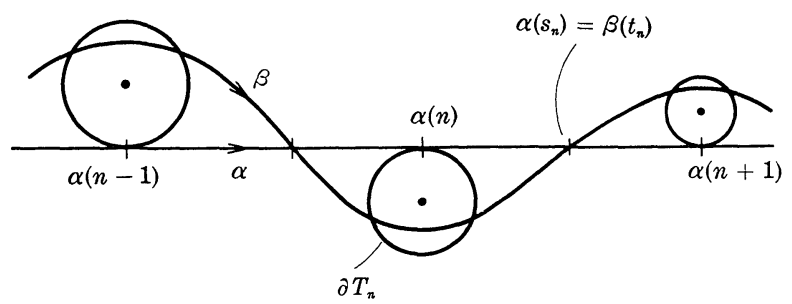

FIGURE 2

By the triangle inequality, the length $t_{n}-t_{n-1}$ of $\beta \mid\left[t_{n-1}, t_{n}\right]$ satisfies

$$
t_{n}-t_{n-1} \leqq s_{n}-s_{n-1}+2^{1-n}
$$

since $\alpha$ is a minimizing geodesic of $M$, summing the inequality above yields $d\left(\beta 0, \beta t_{m}\right)=s_{m} \geqq t_{m}-2$ for all $m$; hence $\beta$ is almost minimizing. But since $t_{n}-t_{n-1}>s_{n}-s_{n-1}, \beta$ is not ultimately minimizing.

We now relate almost minimizing geodesics in $M$ to the horocyclic limit set $L_{h}(D)$.

Lemma 7.3. Let $\alpha$ be a geodesic in $H$ with $\alpha(\infty)=x$. Then $D\left(\alpha t_{0}\right) \cap N\left(\alpha t_{1}, x\right)=\square$ for $t_{0} \leqq t_{1}$ if and only if $d\left(\pi \alpha t_{0}, \pi \alpha t\right) \geqq t-t_{1}$ for all $t$.

Proof. The limit ball $N\left(\alpha t_{1}, x\right)=\bigcup_{t \geqq t_{1}} N_{t-t_{1}}(\alpha t)$. The following 
assertions are equivalent: $D\left(\alpha t_{0}\right) \cap N\left(\alpha t_{1}, x\right)=\square ; D\left(\alpha t_{0}\right) \cap N_{t-t_{1}}(\alpha t)=\square$ for all $t \geqq t_{1} ; d\left(\alpha t, D \alpha t_{0}\right) \geqq t-t_{1}$ for all $t \geqq t_{1} ; d\left(\pi \alpha t, \pi \alpha t_{0}\right) \geqq t-t_{1}$ for all $t \geqq t_{1}$.

A straightforward application of this lemma gives

Proposition 7.4. A point $x \in H(\infty)$ is almost $D$-minimizing if and only if $x \in O_{h}(D)=H(\infty)-L_{h}(D)$, the set of horocyclic ordinary points.

If $H$ satisfies Axiom 1, then $O(D)=H(\infty)-L(D) \subseteq O_{h}(D)$ by Corollary 4.9. For $O(D)$ we obtain the following stronger result.

Proposition 7.5. Let $H$ satisfy Axiom 1. If $x \in O(D)$, the set of cone ordinary points, then $x$ is ultimately D-minimizing.

Proof. We shall prove in Proposition 8.5 that $D$ is properly discontinuous on $O(D)$. Hence we can find a set $U$ containing $x$ which is open in the cone topology and such that $\varphi(U) \cap U=\square$ for every $\varphi \in D$. Let $\alpha \in x$. By Proposition 4.8 there exists $t_{0}>0$ such that $N\left(\alpha t_{0}, x\right) \subseteq U$. Hence $D \alpha\left(t_{0}\right) \cap N\left(\alpha t_{0}, x\right)=\square$. By Lemma 7.3, $d\left(\pi \alpha t, \pi \alpha t_{0}\right) \geqq t-t_{0}$, so $\left.\left.\pi \alpha \mid\right] t_{0}, \infty\right)$ is minimizing.

The converse is false (Theorem 7.10): a limit point can be $D$ minimizing. Note that to prove that $x \in H(\infty)$ is $D$-minimizing it suffices to show that for every geodesic ray $\alpha$ such that $\alpha(\infty)=x$, the geodesic ray $\pi \circ \alpha$ is minimizing in $M=H / D$.

In contrast to the axial case (Proposition 6.7) we do not know if an arbitrary parabolic isometry generates a properly discontinuous group.

REMARK 7.6. (1) If an isometry $\varphi$ of $H$ does not preserve limit spheres at a fixed point $x \in H(\infty)$, then $\varphi$ generates a properly discontinuous group.

(2) Let $\varphi$ be a parabolic isometry that generates a properly discontinuous group. If $\varphi$ has a unique fixed point $x \in H(\infty)$, then for any $p \in H$ the sequences $\left\{\varphi^{n}(p)\right\}$ and $\left\{\varphi^{-n}(p)\right\}$ converge to $x$ as $n \rightarrow \infty$.

Proof. (1) By hypothesis $T_{x}(\varphi) \neq 0$. But $d\left(p, \varphi^{n} p\right) \geqq\left|T_{x}\left(\varphi^{n}\right)\right|=$ $|n|\left|T_{x}(\varphi)\right| \geqq T_{x}(\varphi)$.

(2) In view of Lemma $6.2, x$ is the unique accumulation point of each sequence.

Proposition 7.7. Let $D$ be a properly discontinuous group of isometries, and let $z \in H(\infty)$ be a common fixed point of $D$. Then the following are equivalent. 
(1) $z \in O_{h}(D)$, that is, $z$ is almost $D$-minimizing.

(2) Each element of $D$ preserves limit spheres at $z$.

(3) $z$ is D-minimizing.

Proof. $\quad(1) \Rightarrow(2)$. By hypothesis, given $p \in H$ there exists a limit ball $N$ at $z$ such that $D(p) \cap N=\square$. We must show that $T_{z}(\varphi)=0$ for every $\varphi \in D$. Suppose that $T_{z}(\varphi)=\varepsilon \neq 0$ for some $\varphi \in$ $D$. By changing to $\phi^{-1}$ if necessary we may assume that $\varepsilon<0$. If $f$ is the Busemann function determined by $\gamma_{p z}$, then $f\left(\varphi^{n} p\right)=T_{z}\left(\varphi^{n}\right)=$ $n \varepsilon$ for any integer $n$. Since $N=\{q \in H: f(q)<a\}$ for some number $a, \varphi^{n}(p) \in N$ for a large positive integer $n$. This is a contradiction.

(2) $\Rightarrow$ (3) Let $p \in H$ and let $\alpha$ be the geodesic ray such that $\alpha(0)=p$ and $\alpha(\infty)=z$. Since $\varphi L(p, z)=L(\varphi p, z)=L(p, z)$ for every $\varphi \in D$, we have $D(p) \subseteq L(p, z)$ and $D(\alpha 0) \cap N(\alpha 0, z)=\square$. By Lemma 7.3, $\pi \circ \alpha$ is minimizing.

The proof $(3) \Rightarrow(1)$ is obvious.

Proposition 7.8. If $\varphi$ is a parabolic isometry of $H$, then $\varphi$ preserves limit spheres at least one fixed point $x \in H(\infty)$.

Proof. Let $D$ be the group generated by $\varphi$. If $D$ is not properly discontinuous then by Remark 7.6, $D$ preserves the limit spheres at all fixed points of $\varphi$. Suppose now that $D$ is properly discontinuous. Since $D$ is abelian the displacement function $g_{\varphi}$ is $D$-invariant and induces a smooth convex function $f$ on $M=H / D$ such that $g_{\varphi}=$ $f \circ \pi$. Since $\varphi$ is parabolic, $g_{\varphi}$ has no minimum and neither does $f$. It follows from [3] that there is a geodesic ray $\alpha$ in $H$ such that $\pi \circ \alpha$ is minimizing and $f$ is monotone decreasing on $\pi \circ \alpha$. Thus $g_{\varphi}$ is monotone decreasing on $\alpha$, and $x=\alpha(\infty)$ is a fixed point of $\phi$ that is almost $D$-minimizing. Since $D x=x$ the result follows from Proposition 7.7.

Definition 7.9. A parabolic manifold is a complete $K \leqq 0$ manifold $M=H / D$ such that there is a point $z \in H(\infty)$ that is the unique fixed point of every $1 \neq \varphi \in D$.

Hence every element $1 \neq \phi \in D$ is parabolic. As we shall see in $\S 9$ there are very many such manifolds. Their geometric structure however is rather simple.

THEOREM 7.10. Let $M=H / D$ be a parabolic manifold with $z \in$ $H(\infty)$ the unique fixed point of $D$. Then

(1) $z$ is D-minimizing.

(2) The Busemann function at $z$, unique up to an additive 
constant, induces a continuous convex function $f$ on $M$ which has no minimum.

(3) $M$ is topologically a product $F \times R^{1}$ whose horizontal and vertical fibers are respectively the level surfaces of $f$ and the lines $\pi \circ \alpha$ for all $\alpha \in z$.

Proof. Since $z$ is the unique fixed point of every $1 \neq \varphi \in D$, Proposition 7.8 shows that $\varphi$ preserves limit spheres at $z$. By Proposition 7.7, $z$ is $D$-minimizing, and if $\tilde{f}$ is a Busemann function at $z$ then $\tilde{f}$ is $D$-invariant. Fix the limit sphere $L=\tilde{f}^{-1}(0)$ and let $\eta$ be the projection on $L$. By a remark preceding Proposition 6.6, $\eta$ commutes with every $\varphi \in D$. Thus $\eta$ and $\tilde{f}$ induce maps $\xi$ and $f$ such that the following diagram is commutative. $F$ is the orbit space $L / D$, a topological manifold at least. The function $f$ is convex without minimum on $M$. By

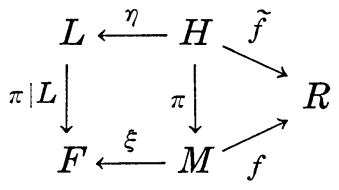

Proposition 3.4 the map $\eta \times \tilde{f}: H \rightarrow L \times R$ is a homeomorphism hence $\xi \times f: M \rightarrow F \times R$ is a homeomorphism. For $t \in R$ the level surface $f^{-1}(t)$ is the projection under $\pi$ of the limit sphere $\tilde{f}^{-1}(t)$. For $p \in$ $L, \eta^{-1}(p)=\alpha(R)$ where $\alpha$ is the unique geodesic in $z$ that meets $L$ at $p$. Hence $\xi^{-1}(\pi p)=\pi \circ \alpha(R)$.

The product decomposition of the preceding theorem resembles that of a negatively curved surface of revolution or, more generally, a warped product $R \times{ }_{f} F$ with $f$ monotone. The principal asymptotes, that is, the elements of $\pi_{a}(z)$, correspond to meridians; the sections $F_{t}=f^{-1}(t)$ correspond to parallels. Other similarities include the following:

(1) Each principal asymptote meets each section orthogonally and exactly once.

(2) The arc length on a principal asymptote of its segment between $F_{s}$ and $F_{t}$ is $|s-t|$.

(3) Any two sections are "parallel" that is $d\left(p, F_{t}\right)=d\left(F_{s}, F_{t}\right)=$ $|s-t|$ for all $p \in F_{s}$.

In this analogy the function $f$ plays the role of distance to the axis to revolution. If $\beta$ is a geodesic of $M, f \circ \beta$ describes how $\beta$ traverses the sections of $M$. If $M$ is a Visibility manifold, then $f \circ \beta$ has a minimum unless $\beta$ is a principal asymptote.

A continuous curve $\alpha:[0, \infty) \rightarrow M$ is divergent if for any compact set $K$ in $M$ there exists $t=t_{K}$ such that for $s \geqq t, \alpha(s) \in M-K$. If 
$\alpha$ is a geodesic then $\alpha$ is divergent if and only if every asymptote of $\alpha$ is divergent. Thus we may speak of divergent and nondivergent asymptote classes. Note that almost minimizing implies divergent. A point $x \in H(\infty)$ will be called $D$-divergent if $\pi_{a}(x)$ is divergent in $M=H / D$.

Our aim now is to show that under mild conditions any parabolic fixed point $x$ is $D$-divergent. This extends a result in [3]. To rigorize the scheme of proof mentioned earlier we define the free displacement function $h_{\varphi}$ of an isometry $\varphi$ of a properly discontinuous group $D$. For $p \in H, h_{\varphi}(p)=\inf \left\{g_{\varphi}(\psi p): \psi \in D\right\}$ where $g_{\varphi}$ is the usual displacement function of $\varphi$. If $\sigma$ is the geodesic segment from $p$ to $\varphi(p)$ then $g_{\varphi}(p)$ is the length of $\pi \circ \sigma$, while $h_{\varphi}(p)$ is the minimum of the lengths of those loops at $\pi(p)$ that are freely homotopic to $\pi \circ \sigma$.

Lemma 7.11. Let $D$ be properly discontinuous and let $1 \neq \varphi \in D$. Then

(1) For any $p \in H$, there exists $\psi$ in $D$ such that $h_{\varphi}(p)=g_{\varphi}(\psi p)$.

(2) $h_{\varphi}$ is uniformly continuous on $H$.

(3) $h_{\varphi}$ is D-invariant.

Proof. (1) Let $\left.r=h_{\varphi}(p)=\inf g_{\varphi}(\psi p): \psi \in D\right\}=\inf \left\{d\left(p, \psi^{-1} \varphi \psi p\right)\right.$ : $\psi \in D\}$. By proper discontinuity there are only finitely many distinct elements $\psi^{-1} \varphi \psi$ such that $d\left(p, \psi^{-1} \varphi \psi p\right) \leqq r+1$. If $\psi^{-1} \varphi \psi$ corresponds to the smallest of these values, then $h_{\varphi}(p)=g_{\varphi}\left(\psi_{p}\right)$.

(2) It suffices to show that $\left|h_{\varphi}(p)-h_{\varphi}(q)\right| \leqq 2 d(p, q)$ for any points $p, q$. Let $p, q$ be given and choose $\psi \in D$ such that $h_{\varphi}(q)=$ $g_{\varphi}(\psi q)$. Then $h_{\varphi}(p)-h_{\varphi}(q) \leqq g_{\varphi}(\psi p)-g_{\varphi}(\psi q)=d\left(\psi p, \varphi_{\psi} p\right)-d\left(\psi q, \varphi_{\psi} \psi\right) \leqq$ $d(\psi p, \psi q)+d(\varphi \psi q, \varphi \psi p)=2 d(p, q)$. Interchanging $p$ and $q$ we obtain the result.

(3) The result is obvious.

In the following we use the terminology of $\S 4$.

Theorem 7.12. Let $H$ be geodesically bounded. Then a fixed point $x$ of a parabolic isometry $\varphi$ is D-divergent for any properly discontinuous group $D$ containing $\varphi$.

Proof. If $x$ is not special then clearly any geodesic in $\pi_{a}(x)$ is divergent in $M=H / D$. If $x$ is special then by Proposition 4.10, $x$ is bounded and there exists $C>0$ such that $d(\gamma, \sigma) \leqq C$ for any geodesics $\gamma, \sigma \in x$.

If $K$ is a compact set in $M$, let $K_{C}=\{q \in M: d(q, K) \leqq 2 C\}$. To prove that every geodesic of $\pi_{a}(x)$ ultimately leaves $K$ it suffices to prove that one geodesic of $\pi_{a}(x)$ ultimately leaves $K_{C}$. Since $K_{C}$ is compact and $h_{\varphi}$ is continuous and $D$-invariant, $h_{\varphi}$ has a minimum 
value $b$ on $\pi^{-1}\left(K_{C}\right)$. By the previous lemma $g_{\varphi}$ assumes the value $b$ at some point, hence there exists $p \in H$ such that $g_{\varphi}(p)<b$ since $g_{\varphi}$ has no minimum. Consider the geodesic ray $\gamma=\gamma_{p x}$. Since $\varphi$ fixes $x, g_{\varphi}$ is monotone decreasing on $\gamma$. If $\gamma(t) \in \pi^{-1}\left(K_{C}\right)$ for some $t \geqq 0$ then $g_{\varphi}(p)<b \leqq h_{\varphi}(\gamma t) \leqq g_{\varphi}(\gamma t) \leqq g_{\varphi}(p)$. This contradiction shows that $\gamma$ does not meet $\pi^{-1}\left(K_{C}\right)$ and hence $\pi \circ \gamma$ does not meet $K_{C}$.

By adding a further restriction we can show that parabolic fixed points are almost $D$-minimizing.

CoROLlaRY 7.13. Let $H$ be geodesically bounded and let $x$ be a fixed point of a parabolic element of $D$. Let $G$ be the subgroup of $D$ consisting of elements that $f x x$ and preserve limit spheres at $x$. If $L / G$ is compact for any limit sphere $L$ at $x$ then $x$ is almost $D$ minimizing.

Proof. Let $\gamma \in x, \gamma(0)=p$. Let $L=L(p, x)$. Since $L / G$ is compact there exists $A>0$ such that for any $q \in L$ there exists $\xi \in G$ such that $d(p, \xi q) \leqq A$. Suppose that $x$ is not almost $D$-minimizing. Then there exist sequences $\left\{\varphi_{n}\right\} \subseteq D$ and $\left\{t_{n}\right\} \subseteq R$ such that $\varphi_{n}(p) \in$ $L\left(\gamma t_{n}, x\right)$ where $t_{n} \rightarrow+\infty$ as $n \rightarrow \infty$. Let $q_{n}=\eta\left(\varphi_{n} p\right)$ where $\eta$ is the projection on ' $L$. Choose $\left\{\xi_{n}\right\} \subseteq G$ such that $d\left(p, \xi_{n}\left(q_{n}\right)\right) \leqq A$. By convexity and the fact that $\eta$ commutes with $\varphi$ we find that $d\left(\gamma t_{n}, \xi_{n} \varphi_{n} p\right) \leqq d\left(\eta \gamma t_{n}, \eta \xi_{n} \varphi_{n} p\right)=d\left(p, \xi_{n} q_{n}\right) \leqq A$. This implies that $\gamma$ is not $D$-divergent which contradicts the preceding theorem.

If $H$ has dimension two then a limit sphere $L$ in $H$ is homeomorphic to $R^{1}$. Thus if $G \neq 1$ then $L / G$ is compact. This need not be the case however in higher dimensions.

8. Trichotomy. As usual $D$ denotes a properly discontinuous group of isometries of $H$, and we use the cone topology on $\bar{H}$ throughout. By Propositions 1.4 and 2.9, the cone limit set $L(D) \subseteq H(\infty)$ is well defined and nonempty when $D$ is nontrivial. By considering the cardinality of $L(D)$, or certain of its subsets, we obtain the trichotomy for Visibility manifolds mentioned in the introduction. This trichotomy will also be described, perhaps more naturally, by the number of equivalence classes of closed geodesics in $M=H / D$.

Let $A(D)$ be the set of all fixed points of axial elements of $D$, and let $P(D)$ be the fixed points of parabolic elements. $O(D)=$ $H(\infty)-L(D)$ is the set of ordinary points.

Proposition 8.1. Let $H$ satisfy Axiom 1. Then

(1) $A(D)$ and $P(D)$ are disjoint countable subsets of $L(D)$. In particular any fixed point of $1 \neq \varnothing \in D$ is in $L(D)$.

(2) $L(D), O(D), P(D)$ and $A(D)$ are each invariant under the 
normalizer of $D$ in the isometry group $I(H)$ of $H$.

Proof. (1) By Propositions 6.4 and 6.7, $A(D) \subseteq L(D) . \quad$ By Theorem 6.5 and a remark following it, $P(D) \subseteq L(D)$. By Proposition 6.8, $P(D)$ and $A(D)$ are disjoint. $P(D)$ and $A(D)$ are countable by Theorem 6.5, since $D \approx \pi_{1}(H / D)$ is countable.

(2) By Propositions 1.4 and 2.9, $L(D)$ and hence $O(D)$ have the invariance property. Let $x$ be a fixed point of an axial [parabolic] element $\varphi$ of $D$. If $\psi \in N(D)$, then $\psi \varphi \psi^{-1} \in D$ fixes $\psi(x)$ and is also axial [parabolic].

We now introduce an important relation, depending on $D$, between points at infinity.

Definition 8.2. Points $x, y$ in $H(\infty)$, not necessarily distinct, are said to be dual relative to $D$ provided that given any neighborhood $U, V$ of $x$ and $y$ respectively in $\bar{H}$ there exists $\varphi \in D$ such that $\varphi(\bar{H}-U) \subseteq V$ and hence $\varphi^{-1}(\bar{H}-V) \subseteq U$.

It is easy to see that the set of points dual to $x \in H(\infty)$ is closed and invariant under $D$. Duality is a symmetric relation.

The following result shows that dual points must both lie in $L(D)$.

Proposition 8.3. If $x, y$ are dual points in $H(\infty)$ then there exists a sequence $\left\{\varphi_{n}\right\} \subseteq D$ such that $\varphi_{n}^{-1}(p) \rightarrow x$ and $\varphi_{n}(p) \rightarrow y$ as $n \rightarrow$ $\infty$ for any point $p \in H$.

Proof. Let $\left\{U_{n}\right\}$ and $\left\{V_{n}\right\}$ be nested local bases at $x$ and $y$ respectively. Let $p \in H$. By duality, for each $n$ there exists $\varphi_{n} \in D$ such that $\varphi_{n}\left(\bar{H}-U_{n}\right) \subseteq V_{n}$. For sufficiently large $n, p \notin U_{n}$ hence $\varphi_{n}(p) \in$ $V_{n}$. Thus $\varphi_{n}(p) \rightarrow y$ as $n \rightarrow \infty$. Similarly we show that $\varphi_{n}^{-1}(p) \rightarrow x$ by using the relation $\varphi_{n}^{-1}\left(\bar{H}-V_{n}\right) \subseteq U_{n}$.

Without some conditions on $H$ there is no guarantee that $x \in$ $L(D)$ has any dual points. For example, if $H=R^{n}$ then no point in $L(D)$ has a dual point. If $H$ satisfies Axiom 1, however, we obtain a converse to the preceding proposition.

Proposition 8.4. Let $H$ satisfy Axiom 1. Let $x, y \in H(\infty)$ and let $\left\{\varphi_{n}\right\} \subseteq D$ be a sequence such that for $p \in H$ we have $\varphi_{n}(p) \rightarrow y$ and $\varphi_{n}^{-1}(p) \rightarrow x$ as $n \rightarrow \infty$. Then $x$ and $y$ are dual. Moreover if $U$ and $V$ are neighborhoods in $\bar{H}$ of $x$ and $y$ respectively, then for $n$ sufficiently large $\varphi_{n}(\bar{H}-U) \subseteq V$ and $\varphi_{n}^{-1}(\bar{H}-V) \subseteq U$.

Proof. We may assume that $V$ is a cone with vertex $p \notin U$. By Proposition 4.7, $\Varangle_{p}\left(\Phi_{n}(\bar{H}-U)\right)=\Varangle_{\varphi_{n}^{-1} p}(\bar{H}-U) \rightarrow 0$ as $n \rightarrow \infty$. Since $\varphi_{n}(p) \in \varphi_{n}(\bar{H}-U)$, the maximum angle at $p$ from $\varphi_{n}(p)$ to $\varphi_{n}(\bar{H}-U)$ 
approaches zero as $n \rightarrow \infty$. Since $\varphi_{n}(p) \rightarrow y$ as $n \rightarrow \infty$, it follows that for $n$ sufficiently large $\varphi_{n}(\bar{H}-U) \subseteq V$.

By this result and the remark following Theorem 6.5 the following consequences are immediate: (1) every point of $L(D)$ has a dual point, (2) the endpoints of an axis are dual, (3) parabolic fixed points are self dual. Further results on duality appear in [7].

Using duality we obtain the following generalization of a well known result of automorphic function theory.

Proposition 8.5. Let $H$ satisfy Axiom 1 and let $D$ be a properly discontinuous group of isometries of $H$. Then the set of points of $\bar{H}$ at which $D$ is properly discontinuous is $H \cup O(D)=\bar{H}-L(D)$.

Proof. By Proposition 1.4, D is not properly discontinuous at any point of $L(D)$. Let $x \in O(D)$, and suppose that $D$ is not properly discontinuous at $x$. If $\left\{U_{n}\right\}$ is a local basis at $x$ then for each $n$ there exists $\varphi_{n} \in D$ such that $\varphi_{n}\left(U_{n}\right)$ meets $U_{n}$. Choosing a subsequence if necessary, let $\varphi_{n}(p) \rightarrow y$ and $\varphi_{n}^{-1}(p) \rightarrow y^{*}$ as $n \rightarrow \infty$. Since $y$ and $y^{*}$ are in $L(D)$ they are distinct from $x$, and we may choose neighborhoods $V, V^{*}$ of $y, y^{*}$ respectively whose closures do not contain $x$. By Proposition 8.4, if $n$ is sufficiently large, then $\varphi_{n}\left(U_{n}\right) \subseteq \varphi_{n}\left(\bar{H}-V^{*}\right) \subseteq$ $V \subseteq \bar{H}-U_{n}$, a contradiction.

The proof of following proposition simplifies a classical argument in automorphic function theory.

Proposition 8.6. Let $H$ satisfy Axiom 1 and let $x, y$ be distinct dual points in $L(D)$. Given any neighborhoods $U$ of $x$ and $V$ of $y$ in $\bar{H}$ there exists an axis of some element in $D$ such that one endpoint is in $U$ and the other endpoint is in $V$.

Proof. We may assume that $U$ and $V$ are cones in $\bar{H}$ whose closures are disjoint. By Corollary 2.12, $\bar{V}$ is a topological $n$-cell. By duality there exists $\varphi \in D$ such that $\varphi(\bar{V}) \subseteq \varphi(\bar{H}-U) \subseteq V$. Hence $\varphi$ has a fixed point in $V$. Similarly $\varphi^{-1}(\bar{U}) \cong U$, so $\varphi^{-1}$ (hence $\varphi$ ) has a fixed point in $U$. The result follows from Proposition 6.4.

We now investigate the cardinalities of various subsets of $H(\infty)$ determined by $D$. The following fact is basic.

Proposition 8.7. Let $H$ satisfy Axiom 1 and let $X$ be a nonempty subset of $H(\infty)$ invariant under $1 \neq \varphi \in D$. Then $X$ consists of one, two or infinitely many points, and if $X$ is finite, $\varphi$ fixes every point of $X$.

Proof. If $X$ is finite, then some power $\varphi^{n}$ fixes every point of 
$X$. Also $D \approx \pi_{1}(H / D)$ is torsion-free. Hence $X$ can contain at most two points, since $\phi^{n} \neq 1$ fixes at most two points. If $X$ consists of one point $x$, then clearly $\varphi$ fixes $x$. Suppose $X=\{x, y\}$. If $\varphi$ fixes either point then it fixes both. If $\varphi$ fixes neither point then it fixes some point $z$ distinct from $x$ and $y$; but then $\varphi^{2}$ fixes $x, y$ and $z$, contradicting Theorem 6.5.

The hypothesis holds, of course, if $X$ is $D$-invariant.

REMARK 8.8. If $H$ satisfies Axiom 1 then the possible cardinalities for an orbit $D(x)$ are $1, \infty$; for $L(D)$ are $1,2, \infty$; for $P(D)$ are 0,1 , $\infty$; for $A(D)$ are $0,2, \infty$.

Proposition 8.9P. If $H$ satisfies Axiom 1 then for any group $D$ the following are equivalent:

(1) $L(D)$ is a singleton, $\{x\}$.

(2) $D$ has a unique common fixed point, $x$.

(3) $H / D$ is parabolic (7.9).

(4) Every $1 \neq \varnothing \in D$ is parabolic.

Proof. $\quad(1) \Rightarrow(2)$. Clearly the uniqe element of $L(D)$ is a common fixed point of $D$, and all fixed points are in $L(D)$ by Proposition 8.1.

$(2) \Rightarrow(3)$. If $1 \neq \phi \in D$ fixes a point $y \neq x$ then by Proposition 6.4, $\varphi$ has an axis joining $x$ to $y$. By Proposition 6.8, $y$ is also a common fixed point of $D$.

$(3) \Rightarrow(4)$. This follows by definition.

$(4) \Longrightarrow(1)$. Let $x \in L(D) . \quad x$ is dual to some point $y \in H(\infty)$ and hence to each point of $D(y)$. Since no axis exists, Proposition 8.6 implies that $y=x$ and $D(x)=\{x\}$. Hence every point in $L(D)$ is a common fixed point of $D$. Again since no axis exists, Proposition 6.4 implies that $L(D)$ is a single point.

Proposition 8.9A. If $H$ satisfies Axiom 1 then the following are equivalent for any group $D$ :

(1) $L(D)$ consists of two points $x$ and $y$.

(2) $D$ has exactly two common fixed points $x$ and $y$.

(3) $H / D$ is axial (6.11) and $D$ is infinite cyclic.

Proof. $\quad(1) \Rightarrow(2)$. This follows from the last assertion of Propositon 8.7.

$(2) \Rightarrow(3) . H / D$ is axial by Proposition 6.4 and infinite cyclic by Proposition 6.11.

$(3) \Rightarrow(1)$. The common endpoints $x$ and $y$ of the axes of $D$ are in $L(D)$. Let $z \in L(D)$, and let $\varphi$ be a generator of $D$. There exists a sequence of integers $\left\{n_{k}\right\}$ such that $\varphi^{n_{k}}(p) \rightarrow z$ as $k \rightarrow \infty$. By Proposi- 
tion $6.7, z$ is either $x$ or $y$.

We emphasize a distinction between the two preceding results: if $D$ consists solely of parabolic isometries they must all have the same fixed point, but if $D$ consists solely of axial isometries they need not have the same fixed points, that is, equivalent axes.

Recall that a complete (nonsimply connected) manifold $M=H / D$ with curvature $K \leqq 0$ is a Visibility manifold if $H$ satisfies Axiom 1 . A Visibility manifold that is neither axial nor parabolic is said to be fuchsian.

Proposition 8.9F. If $H$ satisfies Axiom 1 then the following are equivalent for any group $D$ :

(1) $L(D)$ is an infinite set.

(2) $D$ has no common fixed points.

(3) $H / D$ is fuchsian.

(4) $A(D)$ is infinite.

Proof. In view of the possible cardinalities listed in Remark 8.8, this proposition follows from the two preceding ones and the consequence of Theorem 6.5 that $D$ has at most two common fixed points.

Thus the parabolic, axial and fuchsian types can be distinguished by the number of axes, the number of common fixed points, or the cardinality of the limit set. The first of these criteria can be stated in stronger form as follows:

THEOREM 8.10. Let $M=H / D$ be a Visibility manifold. Then $M$ is parabolic, axial or fuchsian if and only if the number of equivalence classes of closed geodesics in $M$ is 0,1 or o respectively.

Recall that geodesics $\gamma, \sigma$ in $M$ are equivalent if they possess lifts $\widetilde{\gamma}, \widetilde{\sigma}$ in $H$ which join the same points in $H(\infty)$. Thus if $H$ satisfies Axiom 2 as well, then each equivalence class contains a unique geodesic and we may omit "equivalence classes of" from the the statement of the theorem.

Proof. We know that a parabolic manifold contains no closed geodesics and that an axial manifold contains exactly one equivalence class of closed geodesics. Thus it suffices to prove that a fuchsian manifold $M$ contains infinitely many classes.

If an axis of $\varphi \in D$ joins $x$ to $y$, we call $(x, y)$ an axial pair. By Proposition $8.9 \mathrm{~F}$ there is an axial pair $(x, y)$ in $L(D) \times L(D)$, and $D(y)$ contains a point $z$ distinct from $x$ and $y$. It follows from Proposition 6.9 that $(x, z)$ is not an axial pair. Since $x$ and $y$ are dual so 
are $x$ and $z$, and by Proposition 8.6 there exists a sequence $\left\{\left(x_{n}, z_{n}\right)\right\}$ of axial pairs such that $x_{n} \rightarrow x$ and $z_{n} \rightarrow z$ as $n \rightarrow \infty$. Let $A_{n}$ be the set of axes joining $x_{n}$ to $z_{n}$. $\pi\left(A_{n}\right)$ is an equivalence class of closed geodesics in $M$. If $M$ contains only finitely many such classes then we may assume that $\pi\left(A_{n}\right)=A$ for every $n$ by taking a subsequence if necessary. Fix a closed geodesic $\gamma$ in $A$, and let $\alpha_{n}$ be a lift of $\gamma$ in $A_{n}$ for every $n$. In view of Proposition 4.4 we may assume, by taking another subsequence, that $\left\{\alpha_{n}\right\}$ converges to a geodesic $\alpha$ joining $x$ to $z$. Since $\pi \circ \alpha_{n}=\gamma$ for every $n$, we have $\pi \circ \alpha=\gamma$; hence $\alpha$ is an axis. This contradicts the fact that $(x, z)$ is not an axial pair.

From the preceding result and Proposition 6.16 we obtain

COROLlaRY 8.11. If a Visibility manifold $M$ contains either (a) two inequivalent closed geodesics or (b) a closed but not simply closed geodesic, then $M$ is fuchsian and hence contains infinitely many inequivalent closed geodesics.

Part (a) above confirms a suspicion stated in [3] for $K \leqq c<0$.

A geodesic ray $\rho$ in $M=H / D$ is parabolic if $\rho$ has a lift $\tilde{\rho}$ to $H$ such that $\tilde{\rho}(\infty)$ is a parabolic fixed point. In view of Theorem 7.12, a ray $\rho$ in a Visibility manifold $M$ is parabolic if and only if it is divergent and its ray group (cf. $\S 9$ ) is nontrivial, that is, there is a geodesic loop $\alpha_{t}$ at $\rho(t)$ whose length decreases as $t \rightarrow \infty$. In dimension two, one can produce a parabolic ray by adding a contracting tube to a suitable surface. Using Proposition 8.9 we obtain, as suggested in [3], the following complement to Corollary 8.11.

COROLlary 8.12. If a Visibility manifold $M$ contains (a) a parabolic ray and a closed geodesic or (b) two nonasymptotic parabolic rays or (c) two parabolic rays starting at the same point, then $M$ is fuchsian.

Proof. (a) $P(D)$ and $A(D)$ are nonempty.

(b) $P(D)$ is not a singleton.

(c) If the two rays belong to an asymptote class $y \in A(M)$, then $\pi_{a}^{-1}(y)$ is infinite. Hence $P(D)$ is infinite.

We conclude this section with two more characterizations of parabolic, axial and fuchsian manifolds.

An asymptote class $y \in A(M), M=H / D$, is simple if through each point of $M$ there passes a unique element of $y$ (up to parametrization). It is easy to see that $y$ is simple if and only if $\pi_{a}^{-1}(y)$ is a singleton, hence a common fixed point of $D$. Thus the three types of manifolds are characterized by the number of simple classes. 
Proposition 8.13. Let $M$ be a Visibility manifold.

(a) $M$ is parabolic if and only if $M$ has a unique simple class, the principal asymptote class.

(b) $M$ is axial if and only if $M$ has exactly two simple classes, those containing the (oppositely oriented) closed geodesics.

(c) $M$ is fuchsian if and only if $M$ has no simple classes.

We may also describe the types of Visibility manifolds in terms of their ultimately minimizing geodesics.

Proposition 8.14. Let $M$ be a Visibility manifold.

(a) $M$ is parabolic if and only if every geodesic of $M$ is ultimately minimizing.

(b) $M$ is axial if and only if through each point of $M$ there are exactly two geodesic rays that are not ultimately minimizing.

(c) $M$ is fuchsian if and only if through each point of $M$ there are infinitely many geodesic rays that are not ultimately minimizing.

Proof. This follows from Propositions 7.5, 7.7, and 8.9PAF.

9. Fundamental group. The fundamental group $\pi_{1}(M)$ is of obvious importance for a complete $K \leqq 0$ manifold, since the higher homotopy groups of $M$ all vanish. In general the only known algebraic restrictions on $\pi_{1}(M)$ are that it be countable and torsion-free. We shall investigate the fundamental group of a Visibility manifold. The fuchsian type turns out to be the interesting one, and we consider some of its natural subtypes.

The axial type is very special; $\pi_{1}(M)$ is infinite cyclic, and by Corollary 6.16 there are only two diffeomorphism types, $S^{1} \times R^{n-1}$ and $B \times R^{n-2}$, where $B$ is the Möbius band.

The evidence in dimension two suggests that parabolic Visibility manifolds may be rare, since the cylinder $S^{1} \times R^{1}$ is the only diffeomorphism type; furthermore we have seen in Theorem 7.10 that (in any dimension) such manifolds have quite special geometric structure. Nevertheless the fundamental groups of parabolic manifolds form a very large class.

PRoposition 9.1. Let $G$ be the fundamental group of an arbitrary complete $K \leqq 0$ manifold $F$. Then $G$ is the fundamental group of a parabolic Visibility manifold.

Proof. Let $M$ be the warped product $R \times_{\text {exp }} F$. Then $\pi_{1}(M) \approx$ $\pi_{1}(F) \approx G$, and by the curvature formula on page 27 of [3] $M$ has curvature $K \leqq-1$. Since $M$ is complete, it is a Visibility manifold. 
By Lemma 7.8 of [3], exp $\pi$ is strictly convex without minimum on $M$. Hence $M$ has no closed geodesics and must be parabolic.

The fundamental group of every complete $n$-dimensional $K \leqq 0$ manifold occurs as the fundamental group of a complete $(n+1)$ dimensional $K \leqq c<0$ parabolic manifold.

By the remarks above, every two-dimensional Visibility manifold not diffeomorphic to a cylinder or a Möbius band must be fuchsian. In spite of this diversity we shall see the fundamental group of an arbitrary fuchsian manifold must satisfy strong conditions extending results of [3], [5] and [14].

For $H$ and $D$ arbitrary, if $x \in H(\infty)$, denote by $D_{x}$ the stability group $\{\varphi \in D: \varphi(x)=x\}$. These subgroups appear in [3] frequently in an alternative formulation as ray subgroups $\pi_{1}(M, m, \rho)$ of the fundamental group $\pi_{1}(M, m)$. The equivalence of the two is described in and preceding Proposition 10.5 of [3].

For a Visibility manifold $M=H / D$ it follows from earlier results, especially Proposition 6.8, that the non-identity elements of a stability group are either all parabolic or all axial. In the latter case the axes are all equivalent and $D_{x}$ is infinite cyclic. From this viewpoint axial and parabolic Visibility manifolds $H / D$ are exactly those for which $D$ itself is the unique stability group.

We can generalize slightly Corollaries 10.6 and 10.11 and Proposition 10.12 of [3] as follows, omitting the vacuously true axial and parabolic cases:

Proposition 9.2. If $M=H / D$ is fuchsian then

(1) $D \approx \pi_{1}(M)$ is the disjoint (but for $\{1\}$ ) union of its stability groups $D_{x}, x \in H(\infty)$.

(2) Stability groups are permuted by inner automorphisms: $\phi D_{x} \varphi^{-1}=D_{\varphi_{x}}$. Furthermore, $D_{\varphi_{x}}=D_{x}$ if and only if $\varphi \in D_{x}$.

Proof. (1) If $1 \neq \varphi \in D_{x} \cap D_{y}$ then by Proposition 6.4, $\varphi$ translates a geodesic joining $x$ to $y$. Then by Proposition 6.8 we get $D_{x}=$ $D_{y}$, an axial group.

(2) If $\varphi \notin D_{x}$ then the condition $D_{\varphi_{x}}=D_{x}$ implies as in (1) that there exists an axis $\gamma$ from $x$ to $\varphi x$. Since the axes $\gamma$ and $\varphi \circ \gamma$ have the endpoint $\varphi(x)$ in common it follows from Proposition 6.9 that $\gamma$ and $\varphi \circ \gamma$ are equivalent, hence $\varphi^{2}(x)=x$. Since $\varphi$ leaves the set $\{x, \varphi x\}$ invariant, Proposition 8.7 implies that $\varphi$ fixes $x$, a contradiction.

We abstract the major part of these algebraic properties as follows: A disjoint decomposition of a group $G$ is an indexed collection $\left\{G_{i}\right\}$ of subgroups such that

(a) $G=\bigcup_{i} G_{i}$, and for any $i, j$ either $G_{i}=G_{j}$ or $G_{i} \cap G_{j}=1$.

(b) Each $G_{i}$ has strictly disjoint conjugates, that is, if $x G_{i} x^{-1} \cap$ 
$G_{i} \neq 1$ then $x \in G_{i}$.

A group is monic if the only disjoint decomposition it possesses is the trivial one: $G_{i}=G$ for all $i$. Otherwise $G$ is multic.

Elements $x$ and $y$ of a group $G$ are equivalent, written $x \sim y$, provided that for any disjoint decomposition of $G, x$ and $y$ are in the same subgroup $G_{i}$. This is an equivalence relation on $G-\{1\}$.

Lemma 9.3. Let $G$ be a group.

(1) If $x, y$ are elements of $G$ such that $x^{n} y^{p}=y^{q} x^{n}$ for integers $p, q, n$ such that $x^{n} \neq 1, y^{p} \neq 1$, then $x \sim y$.

(2) If $H$ is a monic subgroup of $G$ then for any disjoint decomposition of $G, H$ is contained in a single $G_{i}$.

Proof. (1) There exists a $G_{i}$ such that $y \in G_{i}$ hence $y^{p} \in G_{i}$. Since $x^{n} y^{p} x^{-n}=y^{q}$ and $1 \neq y^{q} \in G_{i}$, it follows from (b) that $x^{n} \in G_{i}$. Since $x^{n} \neq 1, x \in G_{i}$; hence $x \sim y$.

(2) This follows from the fact that if $\left\{G_{i}\right\}$ is a disjoint decomposition of $G$, then $\left\{G_{i} \cap H\right\}$ is a disjoint decomposition of $H$.

Lemma 9.4. A group $G$ with any one of the following properties is monic;

(1) $G$ has nontrivial center.

(2) $G$ is a nontrivial product.

(3) $G$ has a monic normal subgroup $N \neq 1$

(4) $G$ is solvable.

Proof. (1) Let $z \neq 1$ be a central element. If $x$ is any element of $G$, then $x \sim z$ by Lemma 9.3.

(2) Let $\left\{G_{i}\right\}$ be a disjoint decomposition of $G=A \times B$, where $A$ and $B$ are nontrivial. Fix $1 \neq a \in A$, with $(a, 1) \in G_{i}$. Then for any nontrivial $x \in A, y \in B$ by Lemma 9.3 we have $(x, 1) \sim(1, y) \sim$ $(a, 1)$. Thus $(A \times 1) \cup(1 \times B)$ is contained in $G_{i}$, and hence $G=G_{i}$.

(3) Let $\left\{G_{i}\right\}$ be a disjoint decomposition of $G$. Since $N$ is monic $N \leqq G_{i}$ for some $i$. If $x \in G$ then since $N$ is normal in $G$ we have $x G_{i} x^{-1} \cap G_{i} \supseteqq N \neq 1$. Hence $x \in G_{i}$ and $G=G_{i}$.

(4) We prove somewhat more. Suppose there is a (possibly infinite) sequence $H_{0} \subseteq H_{1} \subseteq \cdots \subseteq H_{n} \subseteq \cdots$ of subgroups of $G$ such that (a) $H_{0} \neq 1$ is monic, (b) $H_{n}$ is normal in $H_{n+1}$ for each $n \geqq 0$, and (c) $\cup H_{n}=G$. Then $G$ is monic. This follows readily from (3) and Lemma 9.3(2). It clearly implies (4) since abelian groups are monic.

Further algebraic results about monic groups will appear elsewhere.

By Proposition 9.2 the stability groups $D_{x}, x \in H(\infty)$, constitute a disjoint decomposition of $D$. (Alternatively we may speak of ray sub- 
groups of the fundamental group of $M=H / D$.) If $M$ is axial, then $\pi_{1} M$ is infinite cyclic, hence trivially monic. If $M$ is parabolic it will follow from Proposition 9.1 that $D$ can be either monic or multic. Preceding results yield:

THEorem 9.5. If $M$ is a fuchsian manifold, then its fundamental group $\pi_{1}(M)$ is multic. Furthermore each monic subgroup of $\pi_{1}(M)$ is contained in a single ray group.

We deduce some consequences.

EXAMPLE 9.6. The following groups are multic.

(1) The fundamental group of any compact surface $S$ except the sphere, projective plane, torus and Klein bottle. $S$ admits a Riemannian structure with $K \equiv-1$; hence $S$ is fuchsian and $\pi_{1}(S)$ is multic. These groups have a presentation with a single relation. (The four exceptions have monic fundamental groups by Lemma 9.4(1).)

(2) Any free group $F$ on at least two generators. By attaching contracting tubes to a surface $S$ as above one constructs a fuchsian manifold $M$ with $\pi_{1}(M) \approx F$, at least in the case when $F$ is countably generated.

(3) The fundamental group of the hyperbolic dodecahedral space [15], a 3-dimensional compact manifold with $K \equiv-1$.

Another consequence is a generalization of Preissmann's theorem that a compact manifold with $K<0$ cannot be a nontrivial topological product.

COROLlaRY 9.7. If a non-parabolic Visibility manifold $M$ is homotopically equivalent to a product $P \times Q$ of manifolds, then either $P$ or $Q$ is contractible.

Proof. Since the higher homotopy groups of $M$ vanish so do those of $P$ and $Q$. In the fuchsian case $\pi_{1}(M)$ is multic, so by Lemma $9.4(2)$ either $P$ or $Q$ is simply connected hence contractible. The same must occur in the axial case, since $\pi_{1}(M)$ is infinite cyclic.

REMARK 9.8. A product $M \times N$ of Visibility manifolds need not admit a Riemannian structure making it a Visibility manifold. In fact, if $M$ and $N$ are compact fuchsian manifolds then $M \times N$ cannot be a parabolic Visibility manifold since it is compact and cannot be a nonparabolic Visibility manifold by the preceding corollary.

We give some examples which show in particular $(9.10(2))$ that the conclusion of 9.7 does not hold in the parabolic case even when 
$M$ has constant curvature.

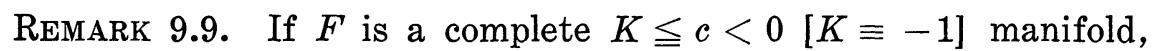
then the warped product $W(F)=R \times_{\cosh } F$ is a complete $K \leqq c<0$ $[K \equiv-1]$ manifold of the same type as $F$ : parabolic, axial or fuchsian. By results in [3] the closed geodesics of $W(F)$ are exactly the closed geodesics of the totally geodesic submanifold $0 \times F=F$. The result then follows from Theorem 8.10.

ExAmple 9.10. Negative Space Forms diffeomorphic to products.

(1) $M$ axial, diffeomorphic to $N \times R^{n}$. With the exception of the Möbius band every axial Visibility manifold is of this diffeomorphism type. To obtain these as space forms set $M=W^{n}(N)$, where $N$ is a circle or a Möbius band with $K \equiv-1 . \quad\left(W^{n}(N)\right.$ is the $n^{\text {th }}$ iterate of $W(N)$ defined above.)

(2) $M$ parabolic, diffeomorphic to a product of nonsimply connected manifolds. Let $A$ and $B$ be nonsimply connected, flat, space forms, and let $M=R \times_{\exp }(A \times B)$. Then $M$ and $W^{n}(M)$ have the required properties.

(3) $M$ fuchsian, diffeomorphic to $N \times R^{n}$. Let $M=W^{n}(N)$ where $N$ is a fuchsian negative space form, for example a double torus.

For examples of $K \leqq-1$ product manifolds that do not admit a complete $K \equiv-1$ metric see [8].

Preissmann in [14] considered manifolds $M=H / D$ for which every element of $D$ is axial. In [3] such an $M$ is called full (there is a closed geodesic in every free class of loops in $M$ ). Axial manifolds are full, but not conversely since every compact $M$ is fuchsian and full. More generally, Proposition 10.15 of [3] asserts that $M$ is full if it contains a compact totally convex set. Generalizing results of Preissmann [14] and Byers [5] on abelian and solvable subgroups respectively we have:

Corollary 9.11. Let $M$ be a full Visibility manifold (for example compact, $K<0$ ). Then every monic subgroup of the fundamental group $\pi_{1}(M)$ is infinite cyclic.

Proof. For a full manifold the disjoint decomposition $\left\{D_{x}: x \in\right.$ $H(\infty)\}$ of $D \approx \pi_{1}(M)$ consists, by Theorem 6.11 , of infinite cyclic groups. But any monic subgroup is contained in some $D_{x}$.

One consequence is that a full Visibility manifold cannot have either a retract or a covering manifold with monic but noncyclic fundamental group. 
Proposition 9.12. Let $H / D$ be a Visibility manifold. If $N$ is a nontrivial normal subgroup of $D$, then $L(N)=L(D)$.

Proof. Clearly $L(N) \subseteq L(D)$, so we prove the reverse inclusion. Let $y \in L(D)$. Choose $p \in H$ and $1 \neq \varphi \in N$. Given any neighborhood $V$ of $y$ in $\bar{H}$ we will find a $\psi \in D$ such that $\psi \varphi \psi^{-1}(p) \in V$. Since $\psi \varphi \psi^{-1} \in N$ it follows that $y \in L(N)$. We can suppose $p \notin V$.

The result is obvious if $H / D$ is parabolic or axial, so we can assume it is fuchsian. Let $y$ be dual to $z$. For some $\eta \in D$ the point $x=\eta z$ is not fixed by $\varphi$ yet dual to $y$. Thus there is neighborhood $U$ of $x$ such that $\varphi U \cap U=\square$. By duality there is a $\psi \in D$ such that $\psi(H-U) \subset V$ and $\psi^{-1}(H-V) \subset U$. Thus $\psi^{-1}(p) \in U$, and hence $\phi \psi^{-1}(p) \in H-U$, which implies $\psi \varphi \psi^{-1}(p) \in V$.

If $\eta: N \rightarrow M$ is a Riemannian covering of a Visibility manifold $M=H / D$, then the Visibility manifold $N$ can be identified with $H / E$, where $E$ is a subgroup of $D$. Furthermore $\eta$ is a regular covering if and only if $E$ is a normal subgroup. Thus the preceding proposition asserts that a nonsimply connected regular covering manifold $N$ of a Visibility manifold $M$ has the same limit set as $M$.

Considering arbitrary (nonsimply connected) coverings, parabolic or axial manifolds can be covered only by manifolds of the same type. A fuchsian manifold $M$ always has coverings by axial manifolds, and if not full, by parabolic manifolds; however the preceding remark shows that any regular covering manifold of $M$ must be fuchsian.

10. Faces and ends. We have seen how to add points at infinity to a Hadamard manifold $H$; now we consider the nonsimply connected case $M=H / D$. For simplicity we assume throughout that $M$ is a Visibility manifold; however much of what we do is valid under weaker hypotheses.

Clearly an arbitrary asymptote class $y \in A(M)$ of $M$ cannot constitute a point at infinity of $M$, since $y$ need not even diverge. We shall define the set $M(\infty)$ of points at infinity of $M$ to be the set of almost minimizing classes in $A(M)$, and as before let $\bar{M}=M \cup M(\infty)$. Note that $M(\infty)$ is empty if and only if $M$ is compact. By Proposition 7.4 the counterimage of $M(\infty)$ under the projection $\pi_{a}: H(\infty) \rightarrow$ $A(M)$ is $O_{h}(D)$. Thus the projection $\pi: H \rightarrow M$ extends in an obvious way to a function, also denoted by $\pi$, from $X=H \cup O_{h}(D)$ onto $\bar{M}$. For any $m \in \bar{M}$ the set $\pi^{-1}(m) \subseteq X$ is an orbit under $D$, hence we may write $\bar{M}=X / D$. Recall that since $M$ is a Visibility manifold, $L_{h}(D) \subseteq L(D)$, hence $O(D) \subseteq O_{h}(D)$. We assign $X$ the topology generated by 
(1) all open sets of $H$;

(2) for each $x \in O(D)$, the intersections with $X$ of all cone neighborhoods of $x$;

(3) for each $x \in O_{h}(D) \cap L(D)$, all augmented limit balls $B^{x}=$ $B \cup\{x\}$ at $x$.

Finally we give $\bar{M}$ the quotient topology derived from the projection $\pi: X \rightarrow \bar{M}$. The space $\bar{M}$ is called the asymptotic closure of $M$. (We shall see in a moment that unlike the special case $\bar{H}, \bar{M}$ need not be compact.) We emphasize that $X$ is in general not a subspace of $\bar{H}$; however $H \cup O(D)$ is an open subspace of both $X$ and $\bar{H}$. Since $O(D)$ and $O_{h}(D) \cap L(D)$ are each invariant under $D$, the space $M(\infty)$ is the disjoint union of its subspace

$$
F(M)=O(D) / D \quad \text { and } \quad V(M)=\left(O_{h}(D) \cap L(D)\right) / D .
$$

By Proposition 8.5, $D$ is properly discontinuous on $H \cup O(D)$, hence the restricted maps $\pi: H \cup O(D) \rightarrow M \cup F(M)$ and $\pi: O(D) \rightarrow F(M)$ are covering maps. In particular, $M \cup F(M)$ is a Hausdorff topological manifold with boundary $F(M)$. We call the connected components of $F(M)$ the faces of $M$; they are topological $(n-1)$-manifolds. By contrast the induced topology on $O_{h}(D) \cap L(D)$, and hence on $V(M)$, is discrete. Points of $V(M)$ are called vertices of $M$. Both faces and vertices are open-and-closed subsets of $M(\infty)$.

EXAmple 10.1. (1) Let $M$ be parabolic with $L(D)=\{z\}$. Then $O(D)=H(\infty)-\{z\} \approx R^{n-1}$, so $M$ has a single face. Since $z \in O_{h}(D)$, $M$ also has a single vertex $v=\pi z$. Using results from $\S 7$ one can show (at least when $H$ also satisfies Axiom 2) that any limit sphere at $z$ is homeomorphic to $O(D)$, and it follows that $\bar{M}$ is homeomorphic to the cone over $F(M)$ with vertex $v$. Thus in particular $\bar{M}$ is compact if and only if $F(M)$ is. When $\operatorname{dim} M=2, \bar{M}$ is a 2-cell with boundary circle $F(M)$; however in higher dimensions $\bar{M}$ is never a manifold with boundary, for since $F(M)$ cannot be a sphere the vertex $v$ does not have Euclidean neighborhoods.

(2) Let $M$ be axial, with $L(D)=\{x, y\}$. Then $O(D)=H(\infty)-$ $\{x, y\} \approx R^{1} \times S^{n-2}$. Since $x, y \in L_{h}(D), M$ has no vertices. If $\operatorname{dim} M=$ $n \geqq 3, M$ has a single face homeomorphic to $S^{1} \times S^{n-2}$ and $\bar{M}$ is homeomorphic to $S^{1} \times E^{n-1}$ ( $E^{n-1}$ the closed $(n-1)$-cell). Thus $\bar{M}$ is a generalized solid torus with boundary $M(\infty)=F(M)$ a generalized torus. In dimension $2, \bar{M}$ is either an ordinary band $S^{1} \times E^{1}$ with faces the two boundary circles, or a Möbius band with its boundary circle the only face. Thus in all cases $\bar{M}$ is a compact manifold with boundary $M(\infty)$.

Note that if $\varphi$ is an isometry of $H$ that preserves $O_{h}(D)$, then its (bijective) extension $\varphi: X \rightarrow X$ is a homeomorphism, since $\varphi$ pre- 
serves cones and limit balls. It follows that the projection $\pi: X \rightarrow$ $\bar{M}$ is open, since for $U \subset X$ we have $\pi^{-1}(\pi U)=D U$.

If $\psi$ is an isometry of $M$ and $\alpha$ a geodesic of $M$, then as before we can define the asymptotic extensions $\psi$ and $\alpha$ to $\bar{M}$, using the same letter to denote asymptotic extensions.

We now verify that the topology of $\bar{M}$ is admissible in the sense defined for $\bar{H}$ in $\S 1$. Explicitly:

Proposition 10. Let $M$ be a Visibility manifold with $\bar{M}$ its asymptotic closure. Then

(1) $M$ is an open dense subspace of $\bar{M}$.

(2) If $\alpha:[0, \infty) \rightarrow M$ is an almost minimizing geodesic of $M$, then the asymptotic extension $\alpha:[0, \infty] \rightarrow M$ is continuous.

(3) If $\psi$ is an isometry of $M$, then the asymptotic extension 广: $\bar{M} \rightarrow \bar{M}$ is a homeomorphism.

(4) If $U$ is a neighborhood of $y \in M(\infty)$ in $\bar{M}$ and $r>0$ is any number, then there is a neighborhood $V$ of $y$ such that $N_{r}(V) \subseteq U$.

Proof. Properties (1), (2), (4) derive in a straightforward way from the admissibility of the cone and horocycle topologies on $\bar{H}$, and the fact that $\pi: X \rightarrow \bar{M}$ is open. To prove (3) it suffices to show that $\psi$ has a continuous extension $\bar{\psi}: \bar{M} \rightarrow \bar{M}$; for $\bar{\psi}$ is then necessarily the asymptotic extension.

Since $H$ is simply connected it is easy to show that there is an isometry $\nu$ of $H$ that covers $\psi$, that is, such that $\pi \circ \nu=\psi \circ \pi$, Then for any $\varphi \in D$ we have $\pi \circ \nu \circ \varphi \circ \nu^{-1}=\psi \circ \pi \circ \varphi \circ \nu^{-1}=\psi \circ \pi \circ \nu^{-1}=\psi \circ \psi^{-1} \circ \pi=$ $\pi$; hence $\nu \circ \varphi \circ \nu^{-1} \in D$. Similarly, since $\nu^{-1}$ covers $\psi^{-1}$ we have $\nu^{-1} \circ \varphi \circ \nu \in$ $D$. Thus $\nu$ is in the normalizer $N(D)$ of $D$ in $I(H)$. By Proposition 1.4, $O_{h}(D)$ is invariant under $\nu$, hence as noted above $\nu: X \rightarrow X$ is a homeomorphism.

We assert that $\pi \circ \nu: X \rightarrow \bar{M}$ is $D$-invariant. In fact, if $\varphi \in D$ then $\nu \circ \varphi=\eta \circ \nu$ for some $\eta \in D$; thus $\pi \circ \nu \circ \varphi=\pi \circ \eta \circ \nu=\pi \circ \nu$. The induced function $\bar{\psi}$ on $\bar{M}$, for which $\pi \circ \nu=\bar{\psi} \circ \pi$, is continuous since $\bar{M}$ has the quotient topology. Now $\psi$ and $\bar{\psi} \mid M$ are equal since both are covered by $\nu$; hence $\bar{\psi}$ is the required continuous extension of $\psi$.

Next we identify the ends of $M$ with certain subsets of $M(\infty)$ and relate them to the faces of $M$. It will be convenient to express the notion of end as follows. Divergent curves $\alpha$ and $\beta$ in $M$ will be called cofinal, written $\alpha \approx \beta$, if given any compact set $K$ in $M$ some final segments $\alpha([s, \infty))$ and $\beta([t, \infty))$ of $\alpha$ and $\beta$ lie in the same connected component of $M-K$. This is clearly an equivalence relation on the set $\Delta M$ of divergent curves in $N$, and the resulting equivalence classes are the ends of $M$.

It is clear that asymptotic divergent geodesics are cofinal. A 
variation of a standard argument shows that there is a minimizing geodesic ray cofinal to any given divergent curve. Thus the relation $\approx$ induces an equivalence relation on $M(\infty)$, and the natural bijection $M(\infty) / \approx \leftrightarrow \Delta M / \approx$ identifies the resulting equivalence classes in $M(\infty)$ with the ends of $M$.

Proposition 10.3. Each face of a Visibility manifold $M$ is contained in a single end.

Proof. We make the following observations: (1) If $K$ is a compact set of $H$, then the closure of $D K$ in $\bar{H}$ is $D K \cup L(D)$. (2) If $C$ is a connected component of $O(D)$, then $\pi(C)$ is an entire component of $F(M)$, that is, a face of $M$.

Let $u$ and $v$ be distinct points in the same face of $M$. By (2) there exist points $x$ and $y$ in the same component of $O(D)$ such that $\pi x=u$ and $\pi y=v$. Since $O(D)$ is open in $H(\infty) \approx S^{n-1}$, its components are path connected. Thus there is a continuous curve $\sigma: I \rightarrow O(D)$ joining $x$ and $y$. Fix a point $p \in H$, and for each $t \in I$ let $\rho_{t}$ be the geodesic from $p$ to $\sigma(t)$. We will show that the (almost minimizing) geodesics $\pi \circ \rho_{0}$ and $\pi \circ \rho_{1}$ are cofinal; then $\pi \rho_{0}(\infty)=u$ and $\pi \rho_{1}(\infty)=v$ are in the same end.

Let $A$ be an arbitrary compact set in $M$. Then there is a compact set $B$ in $H$ such that $D B \supseteqq \pi^{-1}(A)$. Since $\sigma$ lies in $O(D)$, by (1) there is a neighborhood $U$ of $\sigma(I)$ in $\bar{H}$ that does not meet $\pi^{-1}(A)$. If $t \in I$, then since $U$ contains a truncated cone neighborhood of $\sigma(t)$ with vertex $p$, there is a number $r_{t}$ and a neighborhood $N_{t}$ of $t$ in $I$ such that

$$
\text { if } s \in N_{t} \text {, then } \rho_{s}\left(\left[r_{t}, \infty\right)\right) \subseteq U \text {. }
$$

Thus by the compactness of $I$ there is a number $r$ such that $\rho_{t}([r, \infty)) \leqq U$ for all $t \in I$. The function $t \rightarrow \rho_{t}(r)$ is continuous; hence the set $U\left\{\pi \rho_{t}[r, \infty): t \in I\right\}$ is connected, and by construction it does not meet $A$. In paticular $\pi \rho_{0}[r, \infty)$ and $\pi \rho_{1}[r, \infty)$ are in the same component of $M-A$, so $\pi \circ \rho_{0}$ and $\pi \circ \rho_{1}$ are cofinal.

This proposition implies that each end of $M$ is a union of faces and vertices, hence is open and closed in $M(\infty)$. Even if there are no vertices, a single face need not be an end; a manifold with one end consisting of two faces can be constructed by the method of Example 10.6.

Referring to Example 10.1, let $M$ parabolic. If $F M$ is compact, then $M$ has two ends, the face $F M$ and the vertex $v$. Otherwise $M$ has only one end, $M(\infty)=F M \cup\{v\}$. For an axial manifold, faces and ends are the same, with one or exceptionally two of each. 
Since in the parabolic and axial cases there are at most two ends, we have

COROLlaRY 10.4. A Visibility manifold with at least three ends is fuchsian.

(If $M$ has no ends it is compact, hence trivially fuchsian.) Similar but nontopological criteria may be expressed in terms of the number of faces and vertices. For example, a Visibility manifold $\left(\approx S^{1} \times R^{1}\right)$ with at least two faces-or no faces-must be fuchsian.

Corollary 10.5. If a Visibility manifold $M=H / D$ has finite volume, then $M$ has no faces.

Proof. If $M$ has a face, then there is an ordinary point $x \in$ $H(\infty)$. By proposition 8.5 there is a cone neighborhood $U$ of $x$ in $\bar{H}$ such that $\varphi(U) \cap U=\square$ for every $1 \neq \varphi \in D$. Thus the Riemannian covering $\pi: H \rightarrow M$ is injective on $U \cap H$. It is easy to see that $U \cap H$ has infinite volume, hence so do $\pi(U \cap H)$ and $M$.

The converse is false. For example, let $M$ be the double torus with its usual $K=-1$ Riemannian structure, and let $H$ be the commutator subgroup of $\pi_{1}(M)$. The Riemannian covering manifold $M_{I I}$ determined by $H$ may be pictured as the surface of an infinite grid. Since $H$ has infinite index in $\pi_{1}(M), M_{H}$ has infinite area. Because $M$ is compact it follows from Proposition 9.12 that $M_{H}$ has no faces.

We conclude with another method for constructing manifolds with interesting faces and limit sets.

EXAMPLE 10.6. If $M=H / D$ is a complete $K \leqq c<0 n$-manifold we saw in $\S 9$ that $M^{\prime}=R \times_{\cosh } M$ is a complete $K \leqq c<0(n+1)$ manifold of the same type (parabolic, axial, or fuchsian). $M^{\prime}$ is covered by $H^{\prime}=R \times_{\text {cosh }} H$ with projection $\pi^{\prime}=1 \times \pi$, and $H_{0}=\{0\} \times H$ is a totally geodesic copy of $H$ in $H^{\prime}$. By Clairaut methods one can show that every geodesic of $H^{\prime}$ that does not asymptotically approach $H_{0}$ is asymptotic to a geodesic normal to $H_{0}$. Thus $H^{\prime}(\infty) \approx S^{n}$ is separated into two copies of $H$, denoted $H^{-}$and $H^{+}$, by $H_{0}(\infty) \approx H(\infty) \approx$ $S^{n-1}$. Also the action of $D^{\prime}=1 \times D$ on $H^{-}$and on $H^{+}$is essentially that of $D$ on $H \approx H_{0}$. It follows that (1) $L\left(D^{\prime}\right)$ can be identified with $L(D) \subset H_{0}$, hence $\left.O\left(D^{\prime}\right)=H^{-} \cup H^{+} \cup O^{\prime} D\right\rangle ;(2) F\left(M^{\prime}\right)$ is the double of the manifold with boundary $M \cup F(M)$, that is, two copies identified on $F(M)$; and $(3) V\left(M^{\prime}\right) \approx V(M)$.

For instance if $B$ is the punctured torus in Example 11.19, then $F(B)$ is a circle and $F\left(B^{\prime}\right)$ is a double torus.

If $M$ has no faces, so $L(D)=H(\infty)$, then by iterating the operation above $k$ times we get a (fuchsian) manifold $M^{(k)}$ whose limit set 
is, by (1), an $(n-1)$-sphere in the $(n+k-1)$-sphere $H^{(k)}(\infty)$.

Further geometric features of faces and ends appear in the following section.

11. Convex functions. It is known that the convex functions on a manifold $M=H / D$ are significantly related to the geometry of $M$. By linking the convex functions on $M$ to the limit set $L(D)$ we can see in some detail how this comes about. In particular we show that the qualitative character of the convex functions on a Visibility manifold is largely determined by its type (parabolic, axial, or fuchsian).

The totally convex subsets of $M$ contain much the same information as the convex functions. Thus $M$ admits a nonconstant convex function $f$ if and only if $M$ contains a closed totally convex subset $A \neq M$. In fact [3], given $f$ let $A=\{m \in M: f(m) \leqq f(p)\}$ for some $p$, and given $A$ let $f=d(\cdot, A)$.

For a complete manifold $M$ we consider the following natural generalization of compactness: $M$ is core-compact if it contains a (nonempty) compact totally convex subset $A$. Although this property is of interest in general we apply it only in the case of nonpositive curvature, where it is equivalent to the existence of a convex function with compact minimum set. Topologically, the set $A$ is a strong deformation retract of $M$ (Proposition 3.4 of [3]), and since $M$ is full we have seen in $\S 9$ that the fundamental group of $M$ has the generalized Preissmann property. In this section we show that core-compact manifolds have a number of desirable geometric properties.

Recall from [3] that if a convex function is monotone decreasing on a geodesic $\gamma$ of $M$, the same is true for any asymptote of $\gamma$. In particular, it is meaningful to say that a convex function on $H$ is monotone decreasing on $x \in H(\infty)$.

Lemma 11.1. Let $f$ be a convex function on a Hadamard manifold $H$, and let $x \in H(\infty)$. Then $f$ is monotone decreasing on $x$ if and only if there is a sequence $\left\{p_{n}\right\}$ in $H$ such that $\left\{p_{n}\right\} \rightarrow x$ and $\left\{f p_{n}\right\} \rightarrow \inf f$.

Proof. Suppose $f$ is monotone decreasing on $x$ and let $\left\{q_{n}\right\}$ be an arbitrary minimizing sequence, that is, $\left\{f q_{n}\right\} \rightarrow \inf f$. Let $\left\{U_{n}\right\}$ be a nested local basis at $x$. For each $n$, let $\gamma_{n}$ be the geodesic from $q_{n}$ to $x$. Then there exists $t_{n} \geqq 0$ such that $p_{n}=\gamma_{n}\left(t_{n}\right) \in U_{n}$. Since $f$ is monotone decreasing on $\gamma$ we have $f\left(p_{n}\right) \leqq f\left(q_{n}\right)$. Hence $\left\{p_{n}\right\}$ has the required properties.

Conversely, given such a sequence $\left\{p_{n}\right\}$ we show that $f$ is monotone decreasing on $\alpha \in x$. Let $p=\alpha(0)$. For $s \geqq 0$ we must prove $f(\alpha s) \leqq$ 
$f(p)$. Let $\sigma_{n}$ be the geodesic segment from $p$ to $p_{n}$. Since $p_{n} \rightarrow x$, by Proposition 2.14 we get $\sigma_{n}^{\prime}(0) \rightarrow \alpha^{\prime}(0)$, hence $\sigma_{n}(s) \rightarrow \alpha(s)$. (For large $n, \sigma_{n}(s)$ is well defined.) But by convexity $f \sigma_{n}(s) \leqq \max \left\{f(p), f\left(p_{n}\right)\right\}$. Since $f \sigma_{n}(s) \rightarrow f(\alpha s)$ and $f\left(p_{n}\right) \rightarrow \inf f$, we conclude that $f(\alpha s) \leqq f(p)$.

As usual $D$ is a properly discontinuous isometry group on $H$.

LemMa 11.2. Let $f$ be a D-invariant convex function on $H$. If $x \in L(D)$, then $f$ is monotone decreasing on $x$.

Proof Let $\left\{q_{n}\right\}$ be a minimizing sequence for $f$, that is $\left\{f\left(q_{n}\right)\right\} \rightarrow$ $\inf f$. Let $\left\{U_{n}\right\}$ be a nested local basis at $x$ for the cone topology. Since $x \in L(D)$ there exists $\varphi_{n} \in D$ such that $\varphi_{n}\left(q_{n}\right) \in U_{n}$. Thus $\left\{\varphi_{n}\left(q_{n}\right)\right\}$ is a minimizing sequence by the $D$-invariance of $f$, and $\left\{\varphi_{n}\left(q_{n}\right)\right\} \rightarrow x$ by construction. The result then follows from the preceding lemma.

By imposing Axiom 1 we obtain the following basic result used already in $\S 6$.

Proposition 11.3. Let $H$ satisfy Axiom 1. If a convex function $f$ on $H$ is monotone decreasing on distinct points $x, y$ in $H(\infty)$, then $f$ has a minimum. In fact there exists a geodesic $\gamma$ joining $x$ and $y$ such that $\gamma$ is contained in the minimum set $\operatorname{Min}(f)$ of $f$.

Proof. By the preceding lemma there exist minimizing sequences $\left\{p_{n}\right\} \rightarrow x$ and $\left\{q_{n}\right\} \rightarrow y$. If $\sigma_{n}$ is the geodesic segment from $p_{n}$ to $q_{n}$, then Proposition 4.4 implies that, passing to a subsequence if necessary, $\left\{\sigma_{n}\right\}$ converges to a geodesic $\gamma$ joining $x$ and $y$. By convexity $f \circ \sigma_{n} \leqq$ $\max \left\{f p_{n}, f q_{n}\right\}$. Since the latter are minimizing sequences, we conclude that $\gamma \cong \operatorname{Min}(f)$.

CoROLlary 11.4. If $f$ is a convex function on a non-parabolic Visibility manifold $M=H / D$ then any two distinct points of $L(D)$ are joined by a geodesic whose projection lies in the minimum set $\operatorname{Min}(f)$.

Proof. This follows immediately from Lemma 11.2 and Proposition 11.3.

CoROLlary 11.5. A Visibility manifold $M$ is parabolic if and only if $M$ admits a convex function without minimum.

Proof. If $M$ is parabolic its Busemann function is a $C^{1}$ convex function without minimum (Theorem 7.10). If $M$ admits a convex function without minimum, then by the preceding corollary $L(D)$ is a single point and $M$ is parabolic.

Let $f$ be a convex function with minimum on $M$. If $\sigma$ is a geo- 
desic contained in Min $(f)$, then $f$ is trivially monotone decreasing on $\sigma$ and hence on any asymptote of $\sigma$. Also the Busemann function of a parabolic manifold is monotone decreasing on the principal asymptote class. Surprisingly, these are essentially the only ways that the monotone decreasing relation can occur:

Proposition 11.6. Let $f$ be a convex function on a Visibility manifold $M=H / D$. If $f$ is monotone decreasing on a geodesic $\gamma$, then either

(1) $M$ is parabolic and $\gamma$ is a principal asymptote, or

(2) $f$ has a minimum and $\gamma$ is asymptotic to a geodesic $\sigma \leqq$ $\operatorname{Min}(f)$.

Proof. Let $\alpha$ be any lift of $\gamma$ to $H$, so $f \circ \pi$ is monotone decreasing on $\alpha$. Suppose (1) does not hold. Then either $M$ is not parabolic or $M$ is parabolic and $\alpha(\infty)$ is not the unique element of $L(D)$. In either case there exists an $x \in L(D)$ different from $\alpha(\infty)$. By Lemma 11.2 the function $f \circ \pi$ is monotone decreasing on $x$. By Proposition 11.3 there is a geodesic $\beta$ from $x$ to $\alpha(\infty)$ such that $\beta \cong \operatorname{Min}(f \circ \pi)$. Hence $\gamma$ is asymptotic to $\pi \circ \beta \cong \operatorname{Min}(f)$.

We now consider some properties of core-compact manifolds. This class is closed under Riemannian products and Riemannian coverings of finite multiplicity. Parabolic manifolds are never core-compact, since the latter implies full. On the other hand, an axial manifold $M$ is always core-compact since, as we have seen, if $\gamma$ is a closed geodesic of smallest period then $\gamma(R)$ is totally convex.

The following result shows that $f=d(\cdot, \gamma R), \gamma$ as above, is typical of convex functions on $M$ with compact minimum set.

Proposition 11.7. Let $M$ be an axial Visibility manifold and let $g$ be a convex function on $M$ with compact minimum set. Then

(1) $g$ is monotone decreasing on a geodesic $\alpha$ if and only if $\alpha$ is asymptotic to a closed geodesic,

(2) $g$ is constant on a geodesic $\alpha$ if and only if $\alpha$ is equivalent to a closed geodesic,

(3) if Axiom 2 holds, $g$ is constant on $\alpha$ if and only if $\alpha$ is the unique closed geodesic of $M$.

Proof. (1) If $g$ is monotone decreasing on $\alpha$ then $g \circ \pi$ is monotone decreasing on a lift $\beta$ of $\alpha$. Since $g \circ \pi$ satisfies the hypotheses in the lemma below, we conclude that $\beta(\infty) \in L(D)$; that is, $\beta(\infty)$ is an axial endpoint. Hence $\alpha$ is asymptotic to a closed geodesic. The converse is immediate since a convex function is necessarily constant on a closed geodesic. The proof of (2) is similar, and (3) follows from (2). 
The following may be considered a partial converse to Lemma 11.2.

LeMma 11.8. Let $f$ be a convex function with minimum on $H$, and suppose there is a compact set $K$ in $H$ such that $D K \supseteqq \operatorname{Min}(f)$. If $f$ is monotone decreasing on $x \in H(\infty)$, then $x \in L(D)$.

Proof. Let $\alpha$ be a geodesic from a point $p \in \operatorname{Min}(f)$ to $x$. Assume $x \in O(D)$. Thus there is a neighborhood $U$ of $x$ in $\bar{H}$ such that $D(p) \cap U=\square$. By the intensive property of the cone topology there is a neighborhood $V$ of $x$ such that $D K \cap V=\square$. For large $r$, we have $\alpha(r) \in V$; thus $f$ is not monotone decreasing on $\alpha$ and hence not on $x$.

Let $M$ be a complete $K \leqq 0$ manifold. With terminology close to that of [3] we say that an asymptote class $y \in A(M)$ is principal if $y$ is almost minimizing and every convex function on $M$ is monotone decreasing on $y$.

The notion of vertex set defined in $\S 10$ remains valid for $M$, namely $V(M)=\pi_{a}\left(L(D) \cap O_{h} D\right) \subseteq M(\infty)$.

REMARK 11.9. Every vertex of $M$ is a principal asymptote class. This follows immediately from Lemma 11.2 and Proposition 7.4. (See also Proposition 11.15.)

For a parabolic Visibility manifold $M$ the vertex $\pi z$ is the only principal asymptote class, since by Axiom 1 the Busemann function of $M$ is monotone decreasing only on $\pi z$.

Proposition 11.10. Let $M$ be a complete $K \leqq 0$ manifold. Then $M$ is core-compact if and only if $M$ has no principal asymptote classes.

Proof. If $M$ is compact, the assertion holds trivially, so we may assume $M$ is noncompact. If $M$ is core-compact with compact totally convex set $A$, then $f=d(\cdot, A)$ is a convex function that is unbounded on any divergent curve, hence $M$ contains no principal asymptote classes.

Suppose now that $M$ is not core-compact, so every (nonempty) totally convex set is noncompact. We will show that $M$ has a principal asymptote class. Fix $p \in M$, and for each convex function $f$ on $M$ let $K(f)$ be the set of vectors $v \in S(p)$ such that the geodesic ray $\gamma_{v}$ is minimizing and $f$ is monotone decreasing on $\gamma_{v}$. Previous arguments show that $K(f)$ is a closed set; we assert that it is nonempty. By hypothesis the minimum set of $f$ is either empty or noncompact. In either case there exists a divergent sequence $\left\{p_{n}\right\}$ in $M$ such that $f\left(p_{n}\right) \leqq f(p)$. Let $\sigma_{n}$ be a minimizing geodesic segment 
from $p$ to $p_{n}$. Arguing as in the proof of Lemma 11.1 we see that every accumulation point of $\left\{\sigma_{n}^{\prime}(0) \mid n \geqq 1\right\}$ in $S(p)$ lies in $K(f)$.

We must show that some unit vector $v$ is contained in every $K(f)$. Since each $K(f)$ is a closed nonempty subset of the compact space $S(p)$, it suffices to show that $\{K(f): f$ convex on $M\}$ has the finite intersection property. Given convex functions $f_{1}, \cdots, f_{n}$ we set $g=\Sigma e^{f_{i}}$. Then $g$ is a positive convex function, and as shown above there exists a minimizing geodesic ray $\rho$ starting at $p$ on which $g$ is monotone decreasing. Thus each $e^{f_{i}}$, and also each $f_{i}$, is bounded on $\rho$ hence monotone decreasing. Therefore $\rho^{\prime}(0) \in \cap K\left(f_{i}\right)$.

The major argument above makes no use of the curvature hypothesis $K \leqq 0$. In fact, it shows that if an arbitrary complete Riemannian manifold contains no compact totally convex sets then from each point there is a minimizing ray on which every convex function is monotone decreasing.

CoROLlary 11.11. If $M=H / D$ is a core-compact Visibility manifold, then (1) $L_{h}(D)=L(D)$, and (2) every almost minimizing geodesic in $M$ is ultimately minimizing.

Proof. (1) By the preceding Proposition, $M$ has no principal asymptote classes and hence no vertices. But this means that $L(D) \cap$ $O_{h}(D)$ is empty, that is, $L(D) \subseteq L_{h}(D)$. We have seen that the reverse inclusion holds for a Visibility manifold. (2) follows from (1) by the initial results in $\S 7$.

Note that since $M$ has no vertices its asymptotic closure $\bar{M}$ is a manifold with boundary $F(M)$.

Many Riemannian manifolds $N$ do not admit nonconstant convex functions; this is the case if $N$ is compact or more generally if $N$ has finite volume (Proposition 2.2 of [3]). The following result shows there are many complete $K \leqq 0$ manifolds with infinite volume which do not admit nonconstant convex functions (see the example following Corollary 10.5).

Corollary 11.12. If $M=H / D$ is a (complete, $K \leqq 0$ ) manifold with $L(D)=H(\infty)$, then every convex function on $M$ is constant.

Proof. Let $f$ be a convex function on $M$. By Lemma 11.2 the function $f \circ \pi$ is monotone decreasing on every $x \in L(D)=H(\infty)$. Thus $f \circ \pi$ is constant on every geodesic of $H$; hence $f \circ \pi$ and $f$ are constant.

The converse may well be true, at least for Visibility manifolds, however we have been able to prove it (in Proposition 11.15) only for special cases. 
LEMMA 11.13. If $H$ has constant negative curvature or if $H$ is two-dimensional and satisfies Axiom 1, then each point of $H(\infty)$ has arbitrarily small neighborhoods $U$ such that $H-U$ is convex.

Proof. Let $C(v, \varepsilon)$ be a cone neighborhood of $x=\gamma_{v}(\infty)$. By the Visibility property, there exists an $r>0$ such that the open halfspace $U=C\left(\gamma_{v}^{\prime}(r), \pi / 2\right)$ is contained in $C(v, \varepsilon)$. The boundary of $U$ in $H$ is the submanifold $\exp \left(v^{\perp}\right)$ which under the hypotheses on $H$ (though not in general) is totally geodesic. This implies that $H-U$ is convex.

When the conclusion of this lemma holds we obtain the converses of several earlier results, starting with Lemma 11.2:

Lemma 11.14. If $H$ satisfies the conclusion of the preceding Lemma, then $x \in L(D)$ if and only if every $D$-invariant convex function on $H$ is monotone decreasing on $x$.

Proof. It suffices to show that if $x \in O(D)$, then there is a $D$ invariant convex function that is unbounded on $x$. Fix $p \in H$. By hypothesis there is a neighborhood $U$ of $x$ such that $D(p) \cap U=\square$ and $H-U$ is convex. Let $A=\cap\{\varphi(H-U): \varphi \in D\}$. Then $A$ is a $D$-invariant convex set, and $p \in A$. If $\alpha$ is the geodesic from $p$ to $x$, then for large $r$ we have $\alpha(r) \in U \subseteq H-A$. Hence $f=d(\cdot, A)$ is the required function.

Note the following refinement. If $L(D) \neq H(\infty)$, choose such a neighborhood $U_{x}$ for every $x \in O(D)$ and redefine $A$ to be $\cap\left\{\varphi\left(H-U_{x}\right)\right.$ : $\varphi \in D, x \in O(D)\}$. Then $f=d(\cdot, A)$ is unbounded on every $x \in O(D)$, hence the induced convex function on $M=H / D$ is unbounded on every asymptote class $y \in F(M)$, faces of $M$.

The three assertions in the following proposition improve Remark 11.9, Proposition 11.10, and Corollary 11.12 respectively. The assertions are valid for Visibility manifolds of constant curvature or dimension 2, and we consider it likely that they hold for all Visibility manifolds.

Proposition 11.15. Let $M=H / D$ be a Visibility manifold satisfying the conclusion of the preceding lemma. Then

1. The vertices of $M$ are exactly the principal asymptote classes of $M$.

2. $M$ has no vertices (i.e. $L D=L_{h} D$ ) if and only if $M$ is corecompact.

3. $M$ has no faces (i.e. $L D=H(\infty)$ ) if and only if every convex function on $M$ is constant. 
Proof. (1) is clear, since the hypothesis implies the converse of Remark 11.9. Thus (2) becomes a restatement of Proposition 11.10. The hypothesis also shows that if $L(D) \neq H(\infty)$ there is a $D$-invariant convex function on $H$ that is unbounded. Thus the converse of Corollary 11.12 is true, proving (3).

EXAMPLE 11.16. In [4], page 198, Busemann has given two interesting examples of complete $K=-1$ surfaces; see also [7]. Each is diffeomorphically a punctured torus, hence is noncompact with one end. The surfaces are given as $H / D$, where $D$ is a free group generated by two axial isometries with orthogonal axes. Being punctured tori they are necessarily fuchsian $(\S 9)$, however their other geometric properties are quite dissimilar.

(1) Surface $A$ may be visualized as a torus with a contracting tube. $A$ has finite area hence no faces, and thus every convex function on $A$ is constant. $A$ has exactly one vertex $v$, hence $\{v\}$ is the end of $A$; it follows that $v$ is ultimately minimizing. The commutator of the generators is parabolic (with fixed point projecting to $v$ ), hence $A$ is not full. Its asymptotic closure $\bar{A}$ is a torus (add the vertex $v=A(\infty)$ to the end of the tube).

(2) Surface $B$ is a torus with an expanding tube. $B$ has exactly one face, hence infinite area. Examination of a fundamental domain of $D$ shows that $B$ contains a compact totally convex set whose boundary is a closed geodesic (neck of the expanding tube). Thus $B$ is core-compact, full, and has no vertices. Its asymptotic closure $\bar{B}$ is a torus with open dise deleted (add the face $B(\infty) \approx S^{1}$ to the end of the tube).

\section{REFERENCES}

1. D. V. Anosov, Geodesic flows on closed Riemannian manifolds of negative curvature, Proceedings of the Steklov Institute, Number 90.

2. R. Bishop and R. Crittenden, Geometry of manifolds, Academic Press, New York, 1964.

3. R. Bishop and B. O'Neill, Manifolds of negative curvature, Trans. Amer. Math. Soc., 145 (1969) 1-49.

4. H. Busemann, The geometry of geodesics, Academic Press, New York, 1955.

5. W. Byers, On a theorem of Preissmann, Proc. Amer. Math. Soc., 24 (1970) 50-51.

6. J. Cheeger and D. Gromoll, On compact manifolds of nonnegative curvature, preprint (1970), S.U.N.Y. at Stonybrook.

7. P. Eberlein, Geodesic flows in negatively curved manifolds, to appear.

8. - Product manifolds that are not negative space forms, to appear.

9. D. Gromoll and J. Wolf, Some relations between the metric structure and the algebraic structure of the fundamental group in manifolds of nonpositive curvature, preprint (1970), S.U.N.Y. at Stonybrook and U. C. Berkeley.

10. G. Hedlund, The dynamics of geodesic flows, Bull. Amer. Math. Soc., 45 (1939) 241-260. 
11. E. Hopf, Ergodentheorie, Ergebnisse der Mathematik 5, 1937.

12. S. Kobayashi and K. Nomizu, Foundations of Differential Geometry, Wiley (Interscience, New York, 1963, 1969).

13. B. O'Neill, Axioms for Hadamard manifolds, to appear.

14. A. Preissmann, Quelques propriétés globales des espaces de Riemann, Comm. Math. Helv., 15 (1942-43), 175-216,

15. H. Seifert and C. Weber, Die Beiden dodekaeder-räume, Math. Zeitschrift, 37 (1933) 237-253.

Received October 18, 1971. Both authors were partially supported by NSF Grant GP-11476.

University of CAlifornia, Berkeley and Los ANgeles 



\section{PACIFIC JOURNAL OF MATHEMATICS}

\section{EDITORS}

D. Gilbarg and J. Milgram Stanford University

Stanford, California 94305

R. A. Beaumont

University of Washington

Seattle, Washington 98105
J. DugundJI

Department of Mathematics

University of Southern California

Los Angeles, California 90007

RICHARD ARENS

University of California

Los Angeles, California 90024

ASSOCIATE EDITORS
E. F. BECKENBACH
B. H. NeumanN
F. WOLF
K. YoSHIDA

\section{SUPPORTING INSTITUTIONS}

UNIVERSITY OF BRITISH COLUMBIA

CALIFORNIA INSTITUTE OF TECHNOLOGY

UNIVERSITY OF CALIFORNIA

MONTANA STATE UNIVERSITY

UNIVERSITY OF NEVADA

NEW MEXICO STATE UNIVERSITY

OREGON STATE UNIVERSITY

UNIVERSITY OF OREGON

OSAKA UNIVERSITY
UNIVERSITY OF SOUTHERN CALIFORNIA

STANFORD UNIVERSITY

UNIVERSITY OF TOKYO

UNIVERSITY OF UTAH

WASHINGTON STATE UNIVERSITY

UNIVERSITY OF WASHINGTON

$\stackrel{*}{*} \stackrel{*}{*} \stackrel{*}{*}$ AMERICAN MATHEMATICAL SOCIETY

NAVAL WEAPONS CENTER 


\section{Pacific Journal of Mathematics}

\section{Vol. 46, No. $1 \quad$ November, 1973}

Allan Francis Abrahamse, Uniform integrability of derivatives on $\sigma$-lattices .......................................... 1

Ronald Alter and K. K. Kubota, The diophantine equation $x^{2}+D=p^{n} \ldots \ldots \quad 11$

Grahame Bennett, Some inclusion theorems for sequence spaces .......... 17

William Cutler, On extending isotopies ........................ 31

Robert Jay Daverman, Factored codimension one cells in Euclidean

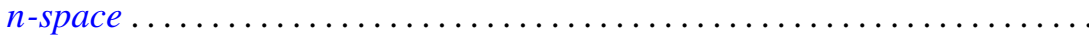

Patrick Barry Eberlein and Barrett O’Neill, Visibility manifolds ............ 45

M. Edelstein, Concerning dentability .......................... 111

Edward Graham Evans, Jr., Krull-Schmidt and cancellation over local

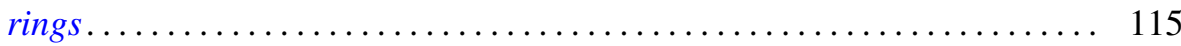

C. D. Feustel, A generalization of Kneser's conjecture ................ 123

Avner Friedman, Uniqueness for the Cauchy problem for degenerate parabolic equations .......................................... 131

David Golber, The cohomological description of a torus action ............ 149

Alain Goullet de Rugy, Un théorème du genre "Andô-Edwards" pour les

Fréchet ordonnés normaux..............................

Louise Hay, The class of recursively enumerable subsets of a recursively enumerable set ........................................

John Paul Helm, Albert Ronald da Silva Meyer and Paul Ruel Young, On orders of translations and enumerations...

Julien O. Hennefeld, A decomposition for $B(X)^{*}$ and unique Hahn-Banach

extensions

Gordon G. Johnson, Moment sequences in Hilbert space .

Thomas Rollin Kramer, A note on countably subparacompact spaces ...

Yves A. Lequain, Differential simplicity and extensions of a derivation ....

Peter Lorimer, A property of the groups Aut $\mathrm{PU}\left(3, q^{2}\right) \ldots$

225

Yasou Matsugu, The Levi problem for a product manifold.

231

John M.F. O'Connell, Real parts of uniform algebras ......

William Lindall Paschke, A factorable Banach algebra without bounded

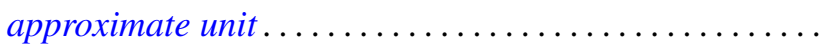

Ronald Joel Rudman, On the fundamental unit of a purely cubic field ....

Tsuan Wu Ting, Torsional rigidities in the elastic-plastic torsion of simply connected cylindrical bars .........................

Philip C. Tonne, Matrix representations for linear transformations on analytic sequences...................................

Jung-Hsien Tsai, On E-compact spaces and generalizations of perfect

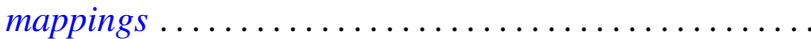

Alfons Van Daele, The upper envelope of invariant functionals majorized by an invariant weight. .. 